\title{
Anhang 2 \\ Paratexte
}

Matthias H. O. Schulz 

Zur Beschreibung von Elementen, die in Manuskripten dem Text beigefügt und neben ihm überliefert, doch nicht sein inhärenter Bestandteil sind, wird der Begriff Paratexte verwendet. Gerade diese Elemente erlauben Rückschlüsse zur Einbettung eines Textes in eine Lebenswirklichkeit zur Zeit der Entstehung seines Trägers und seiner Nutzung über die Zeit. Da Manuskripte kulturhistorische Artefakte sind, die mit bestimmtem Zweck geschaffen wurden, erlaubt eine Erschließung ihrer Texte allein kaum eine Bestimmung ihres Sitzes im Leben. Die handschriftliche Überlieferung des sahidischen Johannesevangeliums erstreckt sich über ein Jahrtausend; es ist natürlich, dass sich der Anteil an nicht dem Text zugehörigen Elementen über die Zeit geändert hat und andere Verwendungen und ein geändertes Textverständnis aufzeigen kann. Neben der zeitlichen muss die geographische Verortung, soweit möglich, beachtet werden. Zwischen dem Weißen Kloster und Deir el-Naqlun liegen ca. 345 km, nach Sakkara sind es knapp $435 \mathrm{~km}$ und zum Makariuskloster gut $525 \mathrm{~km}$ - auf modernen Straßen. Die interpretative Wertung vordergründig identischer Erscheinungen muss immer die Möglichkeit in Betracht ziehen, dass sich an verschiedenen Orten Erscheinungen unabhängig parallel ausgebildet haben.

Die Angaben zu Paratexten verwendeter Handschriften dienen der Dokumentation, um ergänzend zur kritischen Edition des sahidischen Johannesevangeliums Materialien gegliedert und zusammengefasst zu bieten. Alle Manuskripte weisen Besonderheiten auf, die mögliche Verwendungen aufzeigen können. So sind kleinformatige Kodizes für gottesdienstliche Lesungen im kerzenerleuchteten Kirchenraum wegen ihrer meist kleinen Schrift ungeeignet, etwa die Pergamentkodizes 4 und 5 aus dem Jeremias-Kloster oder die Papierhandschriften 19 aus Deir el-Naqlun und 286 aus Deir Abu Makar. Privatgebrauch oder Gebrauch zu Studienzwecken dieser einkolumnigen Kodizes ist denkbar. Für großformatige Kodizes, so 9 und 10 aus dem Michaels-Kloster oder 103, 110, 116, 126, 127, 140, 154 und 234 aus dem Weißen Kloster, ist liturgische Verwendung, zu der das Format gut passt, auch durch Glossen erwiesen. Das Format erlaubt Rückschlüsse zur Verwendung und unter Vorbehalt zur Datierung. Anhand des Formats alttestamentlicher Kodizes und ihrer Einordnung in die Zeit vor und nach der islamischen Eroberung Ägyptens steckte P. Nagel ${ }^{78}$ einen Rahmen ab, um künftig ein Gerüst für paläographische Datierungen zu entwickeln, in den Johannesevangeliumshandschriften nutzbringend einbezogen werden können. Bedacht werden muss indes, dass in literarischen Hand-

78 Nagel 2003: 150-151. 
schriften ältere Schriftformen in weit späterer Zeit nachgeahmt worden sein können, etwa um bessere Lesbarkeit für den liturgischen Vortrag zu erreichen. ${ }^{79}$

\section{Zur Abfolge der mitgeteilten Paratexte}

Makulaturfragmente, die keine wirklichen Paratexte überliefern, sondern zu Reparaturzwecken zufällig einem Kodex beigefügt sind, sind vorangestellt.

Die Handschrift 103 weist mehr nicht textinhärente Elemente als andere Manuskripte auf. Daher steht sie am Beginn der Beschreibungen, gefolgt von überlieferten Kolophonen. Hieran schließt sich ein alphabetisches Verzeichnis der Glossen, liturgischen Vermerke und Titel der Handschriften an, das hernach nach Handschriften aufgeschlüsselt geboten wird.

Die Methoden der Textstrukturierung bilden den Abschluss des Kapitels. Zuerst sind durch Nummerierung gegliederte Einteilungen nach griechischen Kephalaia, nach sahidischen Abschnittsnummern (koptische Kephalaia) und nach Eusebianischen Kanonzahlen Gegenstand, ehe die paratextuellen Aspekte behandelt werden, die zuförderst eine vertiefte Einsicht zur zeitspezifischen Nutzung des Johannesevangeliums in sahidischer Tradition ermöglichen können: Setzungen von Initialen zur Textgliederung stehen auf der Schwelle zwischen textinhärenten und paratextuellen Elementen. Sie an das Ende zu stellen, ist durch die im Vergleich größere Informationsmenge bedingt, die so bessere Übersichtlichkeit erreicht; daher ist sie nicht in andere Abschnitte eingebettet. Da textkritische Symbole nahezu untrennbar mit Initialsetzungen verbunden sind - so Paragraphoi, Obeloi, Diplen oder Koroniden - werden abschließend Initialen mit anderen paratextuellen Elementen in Tabellenform geboten.

Die spezielle Ausformung der Ornamentierungen bei Initialen und der Verzierungen von Paginierungen, Foliierungen und Kopfzeilen sowie Illuminationen werden als primär kunstwissenschaftlich relevante Elemente nicht behandelt. Ebenso werden als kodikologisch bedingte Paratexte Paginierungen, Foliierungen und Kopfzeilen nicht aufgenommen. Perikopenlisten zum Johannesevangelium liegen publiziert vor, weshalb sich eine Wiederholung erübrigt. ${ }^{80}$

Für das sahidische Johannesevangelium bisher singulär sind die $\dot{\varepsilon} \mu \eta \eta \varepsilon \varepsilon i ́ \alpha$

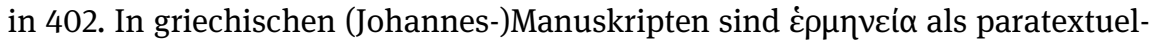
les Element öfter überliefert und werden als „Bibelorakel“ interpretiert. Nach Cirafesi treten sie vornehmlich in zweisprachigen Kontexten auf. ${ }^{81}$

79 E. Wellesz exemplifiziert dies anhand einer griechischen Handschrift (l 1662), siehe Wellesz 1930: 9-24.

80 Siehe Schulz 2018: 220-221; Schulz 2021 und Schulz, Katalog.

81 Cirafesi 2014: 45-67; siehe auch Jones 2014, besonders p. 204-206, 211-214. 


\section{A2.1. Makulaturfragmente}

Beschriftete Makulaturfragmente wurden zumeist zu Reparaturzwecken an Folien angebracht. Die Hinzufügung der Stücke ist bedingt durch den Grad an Schäden eines Manuskripts oder durch die Notwendigkeit, Heft- und Seitenränder zu verstärken. Somit sind enthaltene Texte realiter keine Paratexte, da sie nicht intentionell mit dem Johannesevangelium in Verbindung stehen.

Für die mitgeteilten Texte von Makulaturfragmenten, die eine Lesung erlaubten, sind genauere Studien mit besseren Abbildungen notwendig. SchwarzWeiß und Graustufen-Scans von Mikrofilmen erlauben häufig keine sichere Entscheidung, ob überhaupt Makulaturfragmente mit Schrift vorliegen, weshalb alle Angaben als Anreiz zu weiterer Beschäftigung verstanden werden sollten.

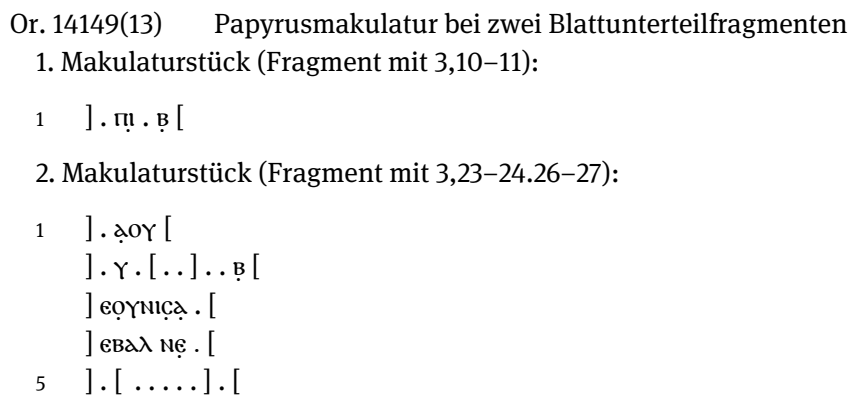

Or. 14149(23) ${ }^{\mathrm{v}} \quad$ Papyrusmakulatur bei Blattunterteilfragment, dokumentarischer Text (Brief), unleserlich und am Original zu prüfen:

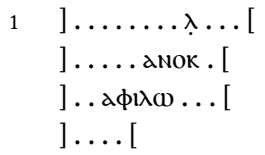

Or. 14149(24) Papyrusmakulatur bei zwei Blattunterteilfragmenten

1. Makulaturstück (Fragmente mit 1,9) schräg an die Seite gesetzt, dokumentarischer Text:

$1 \quad] . \operatorname{lcadK}[$.$] . NHI . [$

] and Na. B...[.] pa [

2. Makulaturstück (Fragment mit 1,22-23.26-27) schräg an die Seite gesetzt, dokumentarischer Text auf $r$ und v sichtbar, unleserlich und am Original zu prüfen:

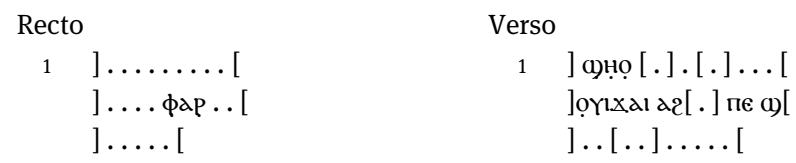


103 O f. $6^{\mathrm{v}} \quad$ [Paris, BnF, Copte 129,10 f. 137] Makulaturstück mit kleinerer Buchschrift, Text unidentifiziert:

$1 \quad$ ]... к़үме $\cdot[$

]анматеүтнс [

] aүmoоne [

]$\lambda \mathrm{Ic}^{\circ}$ Аүвок [

5 ].e.... [

1 statt $\curlyvee$ vielleicht н || 2 emendiere [пр]агматеүтнс || 3-4 recht sicher ist [по] $\lambda \mathbf{c c} z u$ ergänzen, vielleicht auch [єоүпо]入ı, wobei ein Zeilenumbruch nicht sicher vermerkt werden kann.

$S^{\mathrm{r}} \quad$ Makulatur zur Innenrandverstärkung mit Text in Buchschrift, unidentifiziert:

$1 \quad$ ].. [

]peq[

]тơ[ [

]. or[

5 ] 20[

]. Bae[

]Naq[

]$\cdot \overline{\mathrm{N} T}[$

Aus den Buchstabenresten ist in keiner Zeile sicher ein Wort zu ergänzen.

124

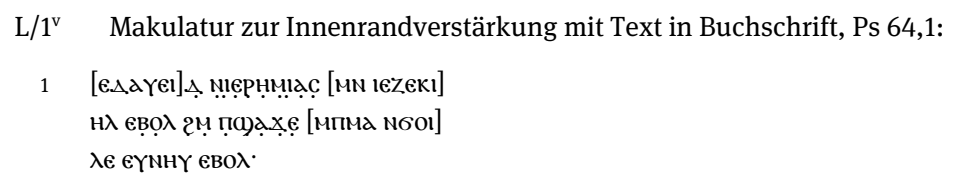

L/1 $\quad$ Makulatur zur Innenrandverstärkung mit Text in Buchschrift, Ps 64,1:

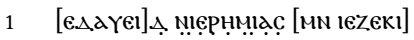

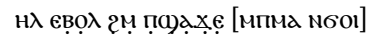

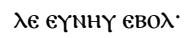

Nach dem Text sind Reste einer Linie erkennbar, was auf eine Abtrennung von Überschrift zu Psalmtext verweisen könnte.

$126 \quad \mathrm{~F} / 1^{\mathrm{r}} \quad$ Makulatur zur Innenrandverstärkung mit Text in Buchschrift, Titel, wohl eines Psalms:

1 T] $] \hat{\mathrm{\omega}}$. [

]пxoẹ[1c

Nach dem Text drei Zierlinien und vielleicht eine weitere, unleserliche Textzeile. Aus dem Psalter kämen für die Abfolge der Wörter Ps 94,1; 95,1; 119,1; 121,1; 122,1; 123,1; 124,$1 ; 125,1 ; 126,1 ; 127,1 ; 129,1 ; 130,1 ; 131,1$ und 133,1 in Frage. 


\section{A2.2. Die Handschrift 103}

Beigefügte Textelemente sind in 103 zahlreicher als in allen anderen Handschriften des sahidischen Johannesevangeliums. Gesonderte Erwähnung verdienen ein Gebet, liturgische Vermerke und der Beginn einer griechischen Perikope auf dem letzten erhaltenen Blatt. Zwar muss offenbleiben, weshalb der Schreiber dem Text des Johannesevangeliums direkt liturgische Angaben und speziell diejenige enthaltene griechische Perikope nachgestellt hat - es wäre zu vermuten, dass er vorderhand verbliebenen Freiraum am Lagenende sinnvoll füllen wollte -, doch lässt sich ableiten, dass 103 bereits bei ihrer Erstellung für liturgische Verlesung konzipiert wurde. 103 ist einer der aussichtsreichsten Kandidaten für einen im Vergleich mit Lektionaren vertieften Einblick in die Vortragsweise von Perikopen im Gottesdienst besonders des Weißen Klosters.

Weitere liturgische Glossen, Kephalaiontitel und Makulaturfragmente sind in den entsprechenden Abschnitten zu Paratexten enthalten und werden hier nicht wiederholt.

Auf den Unterrand von Teil T f. $1^{\mathrm{r}}$ (Oxford, BoL, Clarendon Press, b. 2, Fragm. 8 f. $1^{r}$ ) schrieb eine zweite Hand ein griechisches Gebet. Horner teilt den Beginn mit und gibt пАлакнм дмартолом an, seinerseits wohl als Schreiber des Textes interpretiert. ${ }^{82}$ Schmitz/Mink und Schüssler zitieren den Beginn. ${ }^{83}$

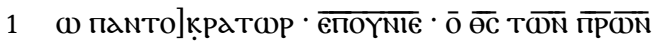
$\pm 9$

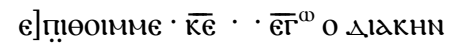
$\pm 7$
] . аммартод̣̣̣...... [..]

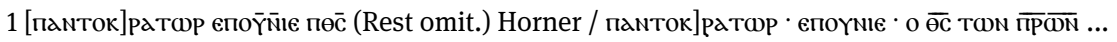

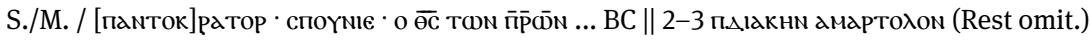
Horner / omit. S./M. / omit. BC.

1 >O Panto]krator, Himmlischer, der Gott der V(ät)er 'w] ünsche, Herr. Ich, der Diakon, ]. . (der) Sünder...... [.. k

Die letzte Folie der Handschrift, Teil U, bietet nach dem sahidischen Text J 21,16-25 auf dem Verso ab Zeile 20 der 1. Kolumne mehrere liturgische Vermerke und den Beginn der Ostersonntagsperikope (20,1-7 $\left.7^{\text {lac }}\right)$ auf Griechisch. Der erste liturgische Vermerk ist von einem Flechtrahmen eingefasst, die weiteren

82 Horner 1911: 379.

83 Schmitz/Mink 1986: 78 und BC 3.2: 45. 
Abschnitte sind durch Zierlinien aus Strichen und Punkten voneinander und vom Folgenden geschieden. ${ }^{84}$ Den griechischen Text zitiert NA 28 als GA 0299 als ständigen Zeugen erster Ordnung. Aland listet den Text ohne Verweis auf das sahidische Manuskript, das ihn enthält, und erwähnt nur die Evangelientexte des Verso. ${ }^{85}$ Die neueste Edition des griechischen Textes bieten Schmid, Elliott und Parker. ${ }^{86}$ Eine Überprüfung zeigte, dass sich nur wenige Verbesserungen der Lesungen von Buchstaben ${ }^{87}$ erreichen lassen, doch bei den Setzungen von Diakritika der koptische Entstehungskontext hilft, manches zu deuten, was von Schmid et al. nicht vermerkt wurde. Insbesondere die Setzung von Supralinearstrichen, die in griechischen Texten nur zur Bezeichnung von Nomina sacra verwendet werden, ist zu erwähnen, da sich so Rückschlüsse zur Aus-

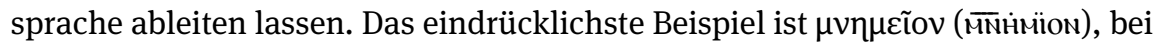
dem die Setzung der Diakritika sehr einheitlich erfolgt. Unzweifelhaft verweist die Setzung von Supralinearstrich, Punkt und Trema darauf, dass der koptische Schreiber das Wort als viersilbig aufgefasst hat, nicht als dreisilbig wie im Grie-

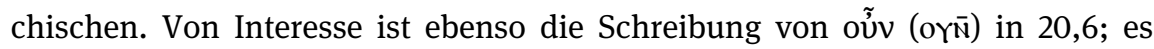
scheint, dass der Kopist an koptisches ơ̄̄ gedacht hat, als er Ny mit einem Supralinearstrich versehen hat. Desgleichen auf einen koptischen Einfluss auf den griechischen Text verweist $\pi \varepsilon$ in 20,5 , das Schmid et al. als Teil von $\mu \varepsilon v \tau o$ ï werten und $\mu \varepsilon v \tau о \ddot{\pi} \varepsilon \varepsilon$ angeben. In NA 28 wird stattdessen $\gamma \varepsilon$ analog zu den Handschriften $L$ und $\Psi$ vermerkt, was im Manuskript nicht zu lesen ist. Tatsächlich handelt es sich um die koptische Kopola nє in einem griechischen Text. In NA 28

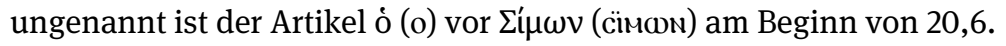

Da der Text des Verso in mehreren Publikationen jeweils nur auszugsweise aufgenommen wurde und für den griechischen Text eine bessere Übersichtlichkeit wünschenswert erschien als in einem Apparat, werden nur Lesungsverbesserungen zu den liturgischen Vermerken als Apparat geboten. An die Edition anschließend ist der griechische Text zeilenweise mit dem der Edition von Schmid et al. und dem Text aus NA 28 abgedruckt; Leitzeile ist die Neuedition.

84 Die liturgischen Vermerke sind in Schmitz/Mink 1986: 79 und BC 3.2: 35, 46 abgedruckt.

85 „J 20,1-7 (kopt. 21,23-25)“, zitiert nach Aland 1994: 44.

86 Edition: Schmid et al. 2007: 167.

87 Als einzig relevante Neulesung ist in 20,1 das zweite c in [ck]otıac zu nennen, dass irrtümlich als Doppelpunkt gedeutet wird. 


\section{sa 103 Uv $^{\mathrm{v}}$ GA 0299 (Paris, BnF, Copte 129,10 f. 199v)}

1

5

10

15

20

25

30

35

40
\{Zeile 1-18

sahidisch J 21,23-25\}

\begin{tabular}{|c|}
\hline 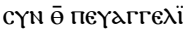 \\
\hline ON חKגTd ÏW \\
\hline $2 \alpha[N]$ NHC : \\
\hline
\end{tabular}

пеYAГ[Ге]入ION NTKY: рїакн м̄пBळ入 N̄N2YM : NIKọץN חкגTa ï : 2ANNHC

NTKYPÏдKH $\triangle \in M \alpha$ :

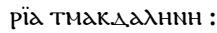

EK TOY KdTd IOWdN .

NHN ariơ EYarгe *

入ioY TO anarnocic :

20,1

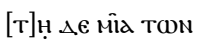

[c]а̣вBdTon - mapï

a. H MаГ $\triangle \partial \lambda$ HNH

ерхетал пров

[ck] otıac etï or
1

5

20,2

10

15

20

$$
20,4
$$

\section{0,5}

30

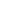

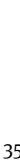

35

20,6
Снс еї то Ммнімї

on - каї в ' $\lambda$ ' епеі том:-

$\lambda \ddot{\theta}$ - HPMENON::

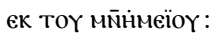

Tpexeï oYN - kaї ẹp

хетаї прос с[1]

мळNa петрос

Каї прос тон $\cdot a \lambda$

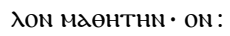

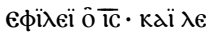

геї аүтої ніраN

TON KYPiON • EK ·

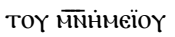

Kaї oYk ö̈АdMEN

пOY EөHкаN aY

20,3 TON - EZH入

оүN' о̃ петрос

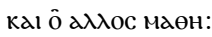

THc. каї нрXоN

Tô eïc Tô MN̄H

Meİọ eтPexon:

$\Delta \epsilon$ ої $\Delta$ Yô оंмоY

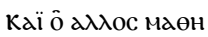

THс проеАрамеN:

Taxeї́n ToY пет

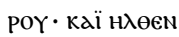

прштос еї то̄

MNнimeїо кaї

паракүYас в $\lambda \epsilon$

пеї тд óөonïa

кeїMeNa - or MEN

тої пє еїсндөєN :

Epxetaï oү̄̄ kaї

ӧ cїmon петрос

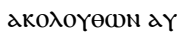

Tw. кaï єїсн $\lambda$

e€N eï то $\overline{M N}$

ḢMєION $\cdot \kappa €$ $\theta €$

opeï та оөonïa

20,7 Kеїmend - каї то

coYAdPION (Textabbruch)

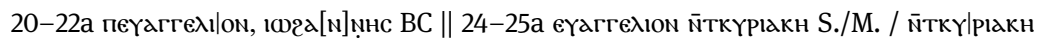

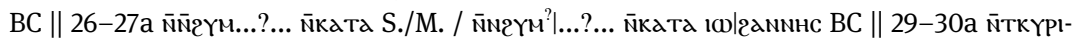

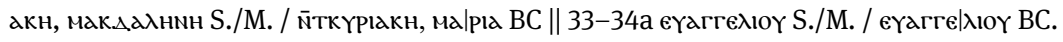




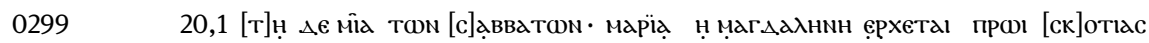

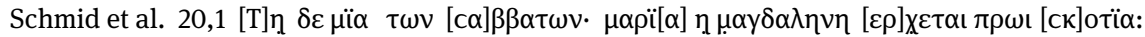

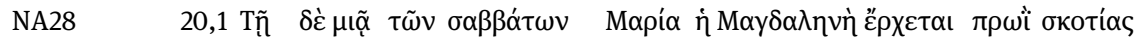

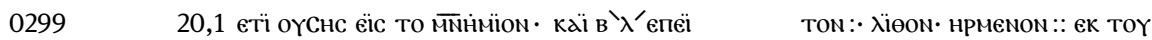

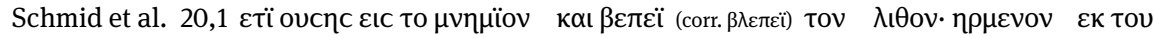

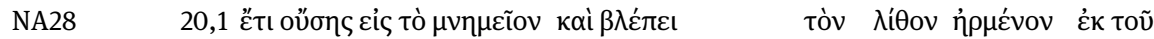

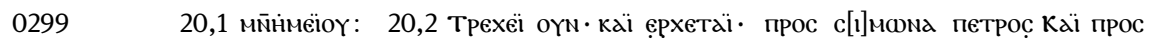

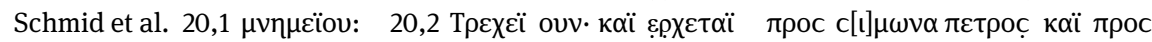

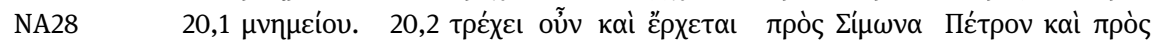

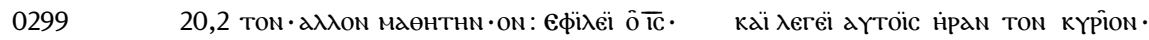

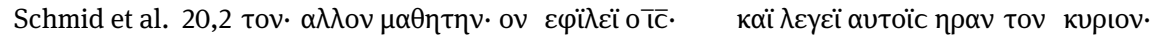

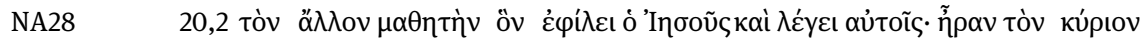

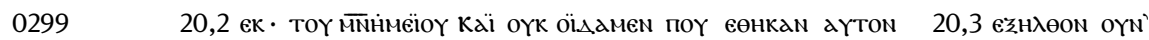

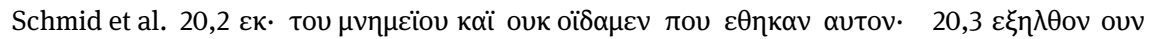

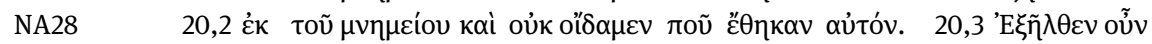

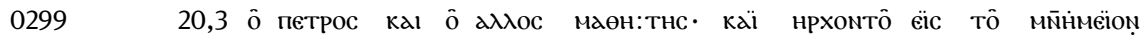

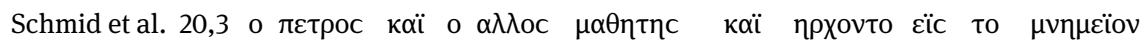

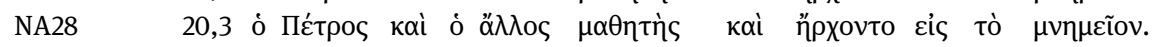

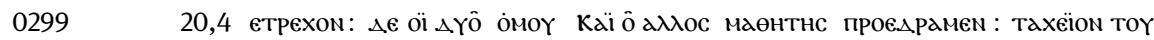

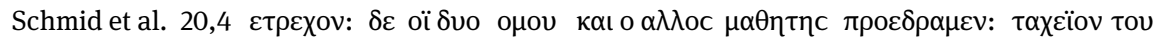

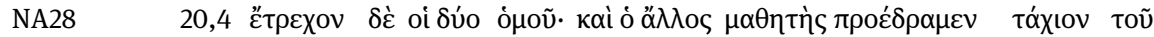

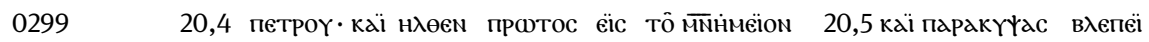

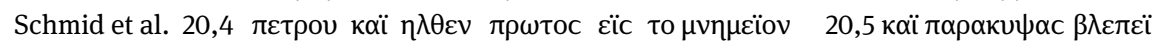

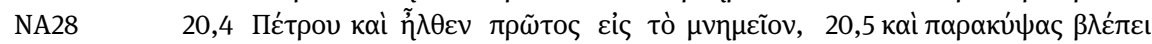

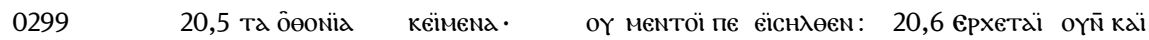

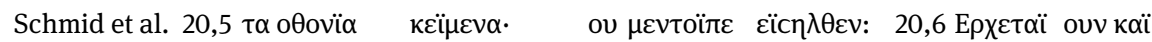

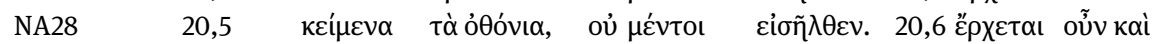

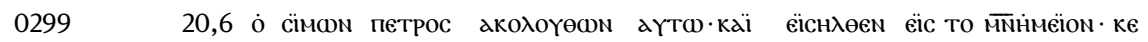

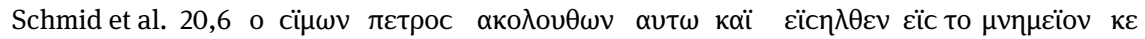

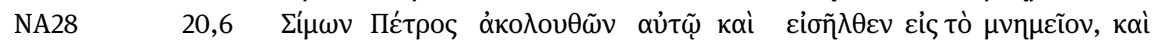

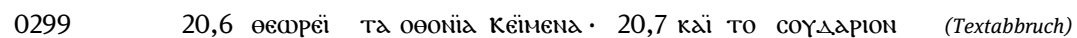

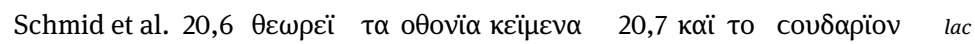

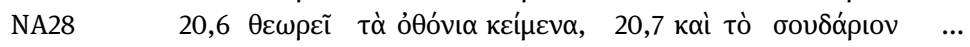




\section{A2.3. Kolophone}

Kolophone sind eine der wenigen Informationsquellen, die etwas über Schreiber, Herkunft einer Handschrift, Umstände der Produktion, ursprüngliche Besitzer und Stifter oder vormoderne Besitzerwechsel verraten können. Um so bedauerlicher ist es, dass für Volltexthandschriften des sahidischen Johannesevangeliums ${ }^{88}$ bisher nur sieben Kolophone bekannt sind $(4,5,10,19,65,82$, $408^{\mathrm{L}}$ ), deren Informationsgehalt dabei ausgesprochen gering ist.

Bis auf die Kolophone von 82 und $408^{\mathrm{L}}$ sind die Texte veröffentlicht, sodass sie hier im Abgleich mit den jeweiligen Publikationen der Vollständigkeit wegen aufgenommen wurden; sie sind in der Folge der SMR-Nummern mitgeteilt. Allen Texten nachgestellt sind Übersetzungen.

4

Der Kolophon wurde von Thompson und Schmitz/Mink mitgeteilt, doch bietet er kaum brauchbare Informationen. Drucktechnisch konnten Tremata in Schmitz/Mink 1986 nicht realisiert werden, was nicht im Apparat vermerkt wird. ${ }^{89}$

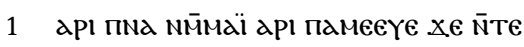

пNOYTE POєIC EPÖ̈ N⿳亠̄̄ TąaH'

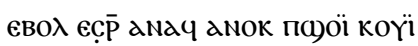

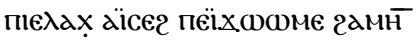

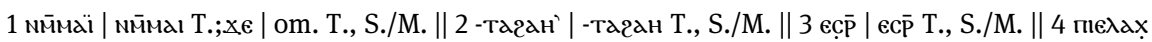

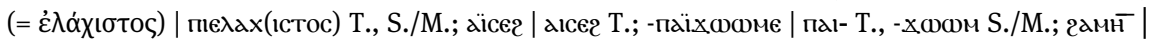
гамн(м) S./M.

1 »Habe Mitleid mit mir und gedenke mein, damit

Gott mich behüte und mein Ende bringe,

wie es ihm gefällt. Ich Pschoi, der Kleine,

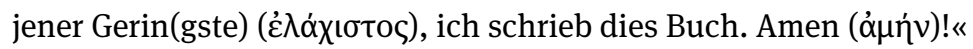

5

Der Kolophon wurde ebenfalls von Thompson, später von Schmitz/Mink und Schüssler veröffentlicht. Bei Schmitz/Mink sind wie bei 4 drucktechnisch Tremata nicht angeführt, was hier im Apparat unerwähnt bleibt. ${ }^{90}$ Auch dieser

88 Kolophone von Lektionaren mit Johannes-Perikopen sind unberücksichtigt. Dies betrifft $15^{\mathrm{L}}$ und 16 ${ }^{\mathrm{L}}$. Für 15 $5^{\mathrm{L}}$ siehe van Lantschoot 1929: 48-49 Nr. 26 und für $16^{\mathrm{L}}$ siehe BC 1.4: 69.

89 Thompson 1932: xvii; Schmitz/Mink 1986: 9.

90 Thompson 1932: xviii; Schmitz/Mink 1986: 12 ; BC 3.1: 38. 
Kolophon bietet keine näheren Informationen über die Handschrift.

1 apl ТАГАПн арl памеEY

E - oyon nim etnaCO)

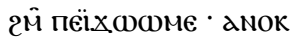

піречрिलове · NंTe пNOY

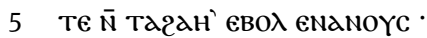

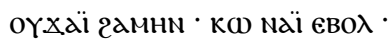

5 -тдгдн' | -тадан Т., S./M, ВC.

1 »Sei so gut (ảyórn) und denke an mich ein jeder, der vorlesen wird aus diesem Buche, mich, jenen Sünder, damit Gott

5 mein gutes Ende bringe.

Lebe wohl, Amen (ảuńv)! Vergib mir!«

10

Der Kolophon der Handschrift ist stark zerstört; nur oberer und unterer Teil sind weitgehend erhalten. Nach einer Trennlinie hat der Priester Gabri (Gabriel) des Michael-Klosters seinen Namen geschrieben. ${ }^{91}$

Ein zweites Blatt mit einem Schenkungsvermerk, das ehemals als Schutzblatt diente, war unter den Fragmenten im Koptischen Museum zu Kairo nicht auffindbar. Da die Abbildungen der Faksimile-Edition keine Textverbesserungen erlauben, wird van Lantschoots Edition wiedergegeben:

I.

$1 \%$ Смоү ёро t十ме

Tanola naeióte

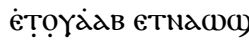

гм пїєаргге

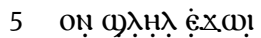

. [ $\pm 8-10 \quad]$.

[ca. 8 Zeilen verloren]

15 . [ $\pm 10-12]$

.. [ $\quad \pm 9-11]$

91 Edition: van Lantschoot 1929: 22-24 Nr. 12. 
ọ [ $[$ 9-11]

Bạ. [ \pm 8-10]

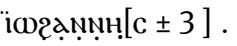

20 plạ.... [ [ 3 ] ọ $\pi$

Alaḳ Baç [. . ]Ү חа

TOYTON еп़וOM

aүo NंTalcealy

ëіо N2үподакако

25 noc пехропос

NंNeMAPTYPOC

$\overline{\phi O H}$

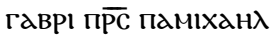

Zierlinie bei v. L. unerwähnt || 1 (vier kreuzförmig angeordnete Winkelhaken) | c c v. L.; epol

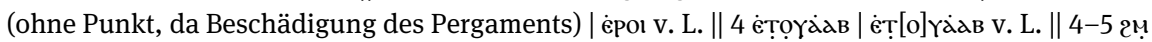

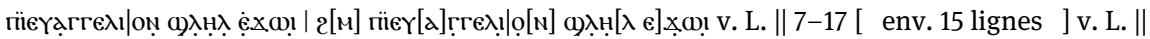

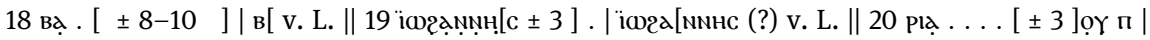

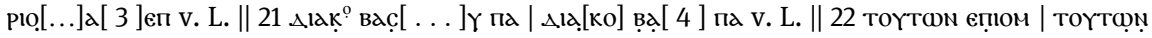

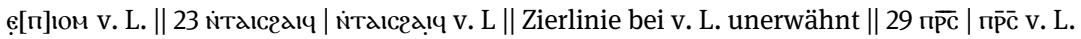

II.

Feuillet de garde, $\mathrm{r}^{\mathrm{0}}$ :

(D) [ $\quad \mathrm{x}$ lignes disparues ]

[ Nсотм етеісмн етмег] мрасе

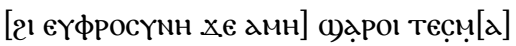

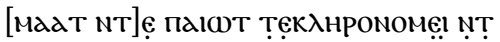

MNTPPO NTAYCeчTOTC Nac XIN T

5 Катаводн мпкосмос гамнN есе

a)लाI

I.

1 ง. »Segnet mich, ich be-

reue ( $\mu \varepsilon \tau \alpha \dot{v} v o เ \alpha)$, meine heiligen

Väter, die vorlesen

aus diesem Evangeli-

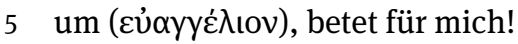

. [ \pm 8-10 ]. .

[ca. 8 Zeilen verloren] 
$15 \cdot[ \pm 10-12]$

.. [ $\pm 9-11]$

? $[ \pm 9-11]$

? [ $\pm 8-10]$

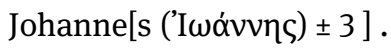

20 ? [ \pm 3$]$ ?

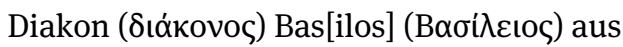

Toutōn im Faijum, und ich schrieb es,

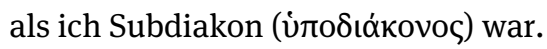

25 Zeit (Хрóvo૬)

der Märtyrer ( $\mu \alpha \dot{\rho} \rho \tau \nu \rho \varsigma$, flektiert: $\mu \alpha \rho \tau u ́ \rho \omega v)$

578

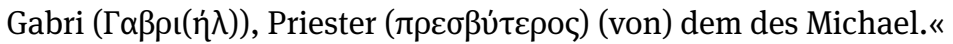

II.

Schutzblatt, recto:

[ x Zeilen ausgefallen ]

[ «zu hören diese Stimme, die erfüllt ist] von Freude

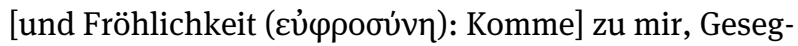

nete meines Vaters, und erbe ( $\kappa \lambda \eta \rho о v o \mu i \alpha)$ das

Königreich, das eingesetzt wurde für sie (lege dich) seit dem

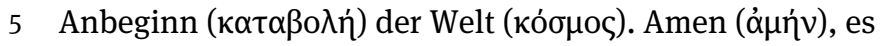
sei! ${ }^{92}$

19

Die Handschrift wird von drei Kolophonen beschlossen, zweien auf Koptisch und einem auf Arabisch. ${ }^{93}$ Die beiden mitgeteilten koptischen Kolophone folgen weitgehend Delattre/Vanthieghem 2016, wurden aber anhand der Abb. gegenkollationiert. Der arabische Kolophon wird hier nicht abgedruckt. ${ }^{94}$

I.

92 Der Text folgt recht wörtlich Mt 25,34. Ich danke J. van der Vliet, Gutachter dieses Bandes, für Hinweise zur Übersetzung. Ohne Emendation ist auch eine Paraphrase denkbar: „Komme] zu mir, Gesegnete meines Vaters und Erbin des Reiches, das eingesetzt wurde für sie seit dem Anbeginn der Welt." Wegen der fehlerhaften Sprache ist die Übersetzung unsicher.

93 Editionen: Delattre/Vanthieghem 2016: 62-67 mit Abb. 1-2.

$94 \mathrm{Zu}$ ihm siehe Delattre/Vanthieghem 2016: 68 mit Abb. 2. 


\section{(Zierleiste)}

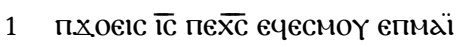
NOYTe NCON NंTaч4I проOҮ() $\bar{M}$

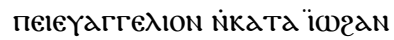

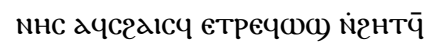

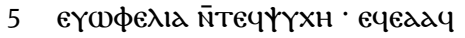
мंм̄пळа тоүсгаї м̈пєчраN еп

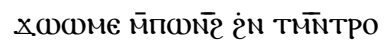

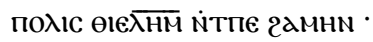
(Zierleiste)

†ромпе таl сарагеNoY vac. $\overline{\text { YГ }}$

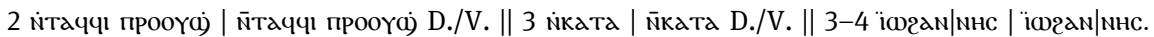

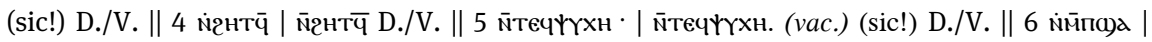

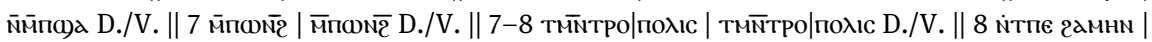

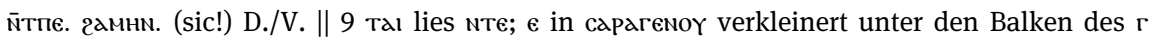
gesetzt und or verkleinert ober- und unterhalb des $\mathrm{N}$ geschrieben.

II.

[ ca. 4-6 Zeilen verloren ]

1 T[ ca. $\pm 16-20$ ]

ePẹ[ ca. $\pm 14-18$

$\mathrm{M}[\quad$ ca. $\pm 16-20$ ]

dpı п[меєеүє са. \pm 5-9] . [ са. \pm 2-4 ]

5 є nс[воүг є] т़сапеснт [нсвоү (?)]

NI[M] петеч: емпо) аN M.

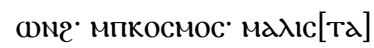

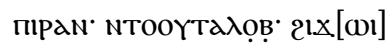

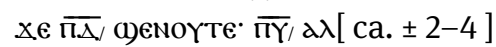

10 ете іодаnе• пє: па пгнт: п. [ са. $\pm 2-4$ ]

w neqlat nd пcd 2HT: or[ ca. $\pm 3-5]$

TE cloY: mecope $N \phi \epsilon / \cdot \omega) \lambda н[\lambda \in x]$

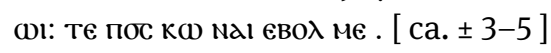

वMHN

4 Buchstabenrest gegen Ende der Zeile wohl von e || 13 über noc ein Supralinearstrich, was sich im Druck nicht nachbilden lässt; Buchstabenrest gegen Ende der Zeile vielleicht von п.

I.

(Zierleiste)

1 »Herr Jesus Christus (Xpıбтóৎ) segne den gott- 
liebenden Bruder, der Sorge trug um

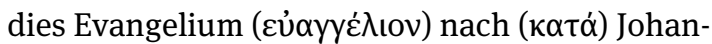

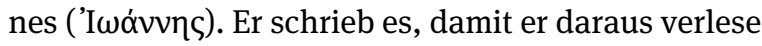

5 zum Nutzen ( $\dot{\varphi} \varphi \dot{\lambda} \lambda \varepsilon \iota \alpha)$ seiner Seele ( $\psi v \chi \eta \dot{)})$, ihn

würdig machend, dass sein Name eingeschrieben werde

ins Buch des Lebens im Königreich

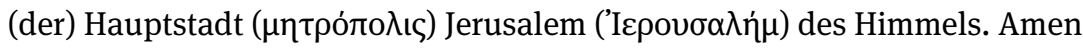
$(\alpha \dot{\alpha} \mu)$ )!

(Zierleiste)

Das Jahr der Sarazenen 493.«

II.

[ ca. 4-6 Zeilen verloren ]

1 ? [ ca. $\pm 16-20$ ]

? [ ca. $\pm 14-18$ ] ]

? [ ca. $\pm 16-20$ ]

»Ged[enke ca. \pm 5-9] . [ ca. \pm 2-4 ]

5 der S[chüler, der] Niedrigste all[er Schüler (?)],

jener, der unwürdig ist $\mathrm{d}$ [es]

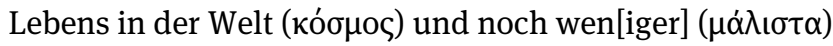

des Namens, den man [ihm au]ferlegt hat:

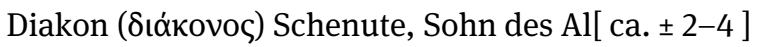

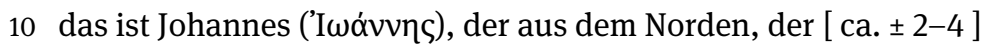

seine Väter aus dem Norden. Ein (?) [ ca. $\pm 3-5$ ]

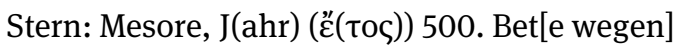

mir: Herr, vergib mir (?) [ ca. $\pm 3-5$ ]

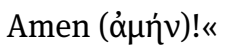

65

Die Lesung folgt weitgehend Urbaniak-Walczak, von der diejenigen Ergänzungen übernommen wurden, die als recht sicher gelten können. ${ }^{95}$ Dankenswerterweise überließ W. Godlewski ein Foto, um Urbaniak-Walczaks Lesungen zu prüfen. Es zeigte sich, dass Lacunae durchweg größer sind, als die editio princeps vermerkt. Bei einer Breite von *ca. $20 \mathrm{~cm}$ für das Blatt, wären in den ersten beiden Zeilen wenigstens 16-17 ausgefallene Buchstaben anzunehmen. Ferner sind einige Buchstabenreste vorhanden, die Urbaniak-Walczak nicht vermerkt.

95 Edition: Urbaniak-Walczak 2006: 998-999. Für weitere Ergänzungsvorschläge und ihren Kommentar siehe Urbaniak-Walczak 2006: 998-1007. 


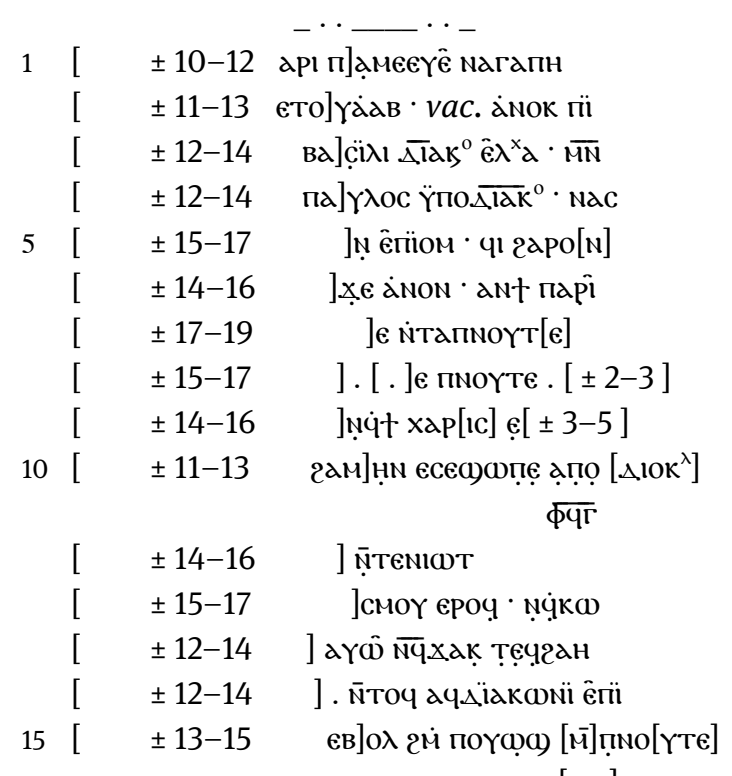

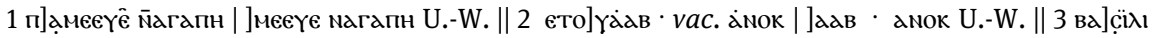

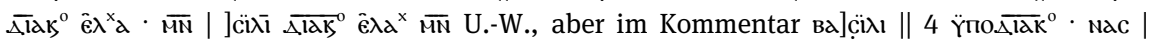

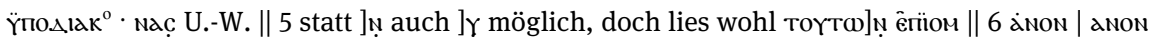

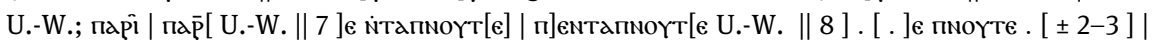

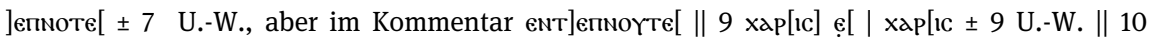

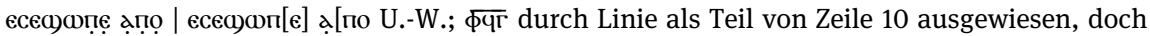

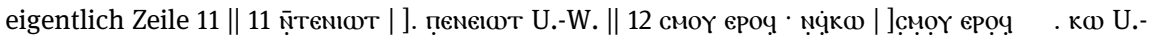

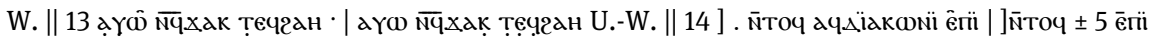
U.-W. || 16 поүळ) | поү(ب) U.-W.

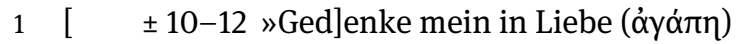

[ $\pm 11-13 \mathrm{der} / \mathrm{die} /$ das hei]lig ist. vac. Ich, jener

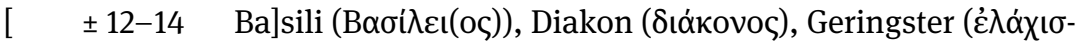

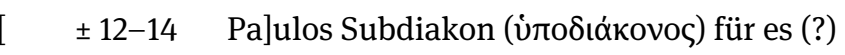
Tos) und

5 [ $\pm 15-17$ Toutō]n im Faijum. Halten [wir] Stand

[ $\pm 14-16 \quad]$ : Wir, wir geben (?)

[ $\pm 17-19$ ] (?) von Got[t]

[ $\pm 15-17 \quad] .[].(?)$ Gott $[ \pm 2-3]$

[ $\pm 14-16$ ] und er gebe Gna[de] (xápıc) für [ $\pm 3-5$ ]

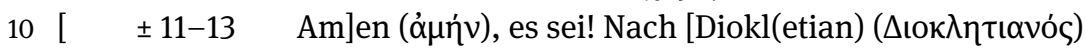




\begin{tabular}{|c|c|c|}
\hline[ & $\pm 14-16$ & ] unseres Vaters \\
\hline & $\pm 15-17$ & ] segne ihn und (er) lasse (vergebe) \\
\hline & $\pm 12-14$ & ] und er vervollkommne sein Ende. \\
\hline & $\pm 12-14$ & 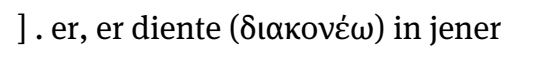 \\
\hline 15 & $\pm 13-15$ & $\begin{array}{l}\text { na]ch dem Wunsch }[\mathrm{G}] \mathrm{ott}[\mathrm{es}] \ll \\
\cdots \ldots \ldots \ldots \\
\cdots\end{array}$ \\
\hline
\end{tabular}

82

Der fragmentierten letzten Textseite folgt auf der Rückseite $(\rightarrow)$ ein Text in kursiver Hand, wohl ein Kolophon. Maximal sind ca. 10,2-10,5 × 12,3-12,6 cm der ursprünglichen Seitenabmessung von ca. 22,5-23,0 × 19,0-19,5 cm erhalten. Zumeist ist aufgrund unregelmäßiger Abbrüche weit weniger Material vorhanden. Da beim Johannestext innere und äußere Kolumne partiell in voller Breite erhalten sind, nach dem erhaltenen Text ein Blattunterteil vorliegt, aber innerer, äußerer und unterer Rand verloren sind, dürfte beim Kolophon bei Maximalausdehnung ein geringer Teil der Zeilenanfänge und -enden abgebrochen sein, doch gänzlich ein größerer Teil des Anfangs und ein kleinerer des Endes. Erhalten sind Reste von ca. 14-15 Zeilen mit ca. 3-24 Buchstaben pro Zeile; Zeile 9 und 10 bieten annähernd den Gesamtbestand an Buchstaben. Der Text ist unpubliziert, doch so schlecht erhalten, dass von einer Edition abgesehen wird, die anhand des Originals erfolgen muss. Erwähnt sei aus Zeile 11 м]ақ̆аріос фоввамом, was vielleicht auf den Herkunftsort verweisen könnte.

$408^{\mathrm{L}}$

Der Kolophon ist Teil der scriptio superior eines in Zweitverwendung für eine Abschrift des Johannesevangeliums verwendeten Pergamentpalimpsests und wird hier erstmals publiziert. Vom Evangelientext der scriptio superior sind bisher keine weiteren Teile bekannt geworden; außer dem Kolophon bietet die Folie nur die Subscriptio. Die scriptio inferior gehört zu einem Lektionar, unter dessen Perikopen ebenfalls das Johannesevangelium vertreten ist.

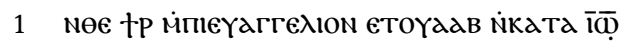

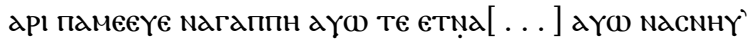

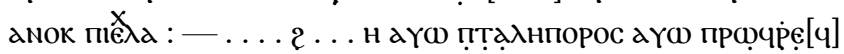

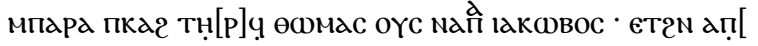

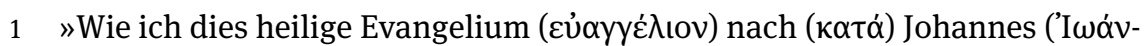
vnऽ) erstellte, gedenke mein in Liebe (ảyórn) und der, die wird [ . . . ] und meinen Brüdern. 


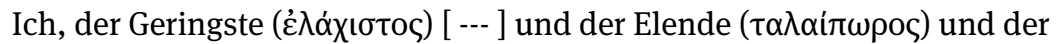

Abschaum

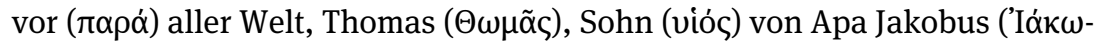
ßos), der in [ « 


\section{A2.4. Übersicht zu Glossen, liturgischen Vermerken und Überschriften}

Neben Zahlangaben sind Glossen das häufigste paratextuelle Element. Fast immer stammen sie von zweiter bzw. späterer Hand und sind meist liturgisch oder inhaltsbeschreibend. Liturgische Glossen bzw. Vermerke oder Rubriken bieten Informationen zur Nutzung, inhaltsbeschreibende Glossen zum Inhalt einer Textpassage. Oft sind inhaltsbezogene Angaben auch liturgisch interpretierbar.

Überschriften sind außer bei Inscriptiones nur in Lektionaren belegt. Das Johannesevangelium ist einer der Texte, der in der koptisch-orthodoxen liturgischen Tradition weitreichende Verwendung genießt. Als liturgische Lesung sind Abschnitte sowohl zu Hochfesten als auch zu gewöhnlichen Anlässen des Kirchenjahres heute und in der Vergangenheit belegt. Evangelienlesungen stehen normal nicht am Beginn einer Perikopenfolge, weshalb Überschriften zum Johannesevangelium den Anlass ihrer Verwendung gewöhnlich nicht nennen. ${ }^{96}$ Neben Lektionaren und anderen Manuskripten, die direkt auf eine liturgische Verwendung verweisen, erlauben Volltexthandschriften aufgrund von Glossen und textkritischen Zeichen Rückschlüsse zum Umfang liturgischer Lesungen. Insbesondere die Handschriften 102 und 124 weisen Symbole auf, die sicher Perikopenbeginn und -ende angeben:

102 Ornamentierte Initialen, Paragraphoi und modifizierte Paragraphoi, die am ehesten

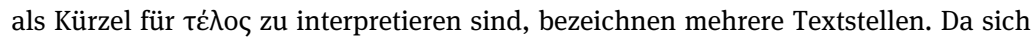
durch Vergleiche zeigen lässt, dass in zwei Fällen Perikopenanfänge bezeichnet werden, handelt es sich recht sicher um Verweise auf Perikopenanfänge und -enden.

$\mathrm{P}^{\mathrm{r}} \quad$ (4,24, Desinit) Ende durch modifizierten Paragraphos bezeichnet.

$\mathrm{Q}^{\mathrm{v}}-\mathrm{R}^{\mathrm{r}}$ (K 9215-K 9076; 9,24-38) Beginn durch ornamentierte Initiale, Ende durch modifizierten Paragraphos bezeichnet.

$\mathrm{R}^{\mathrm{v}} \quad(10,1-5)$ Beginn durch ornamentierte Initiale, Ende durch modifizierten Paragraphos bezeichnet, vergleiche 103 „Für einen Erzbischof“.

$\mathrm{R}^{\mathrm{v}} \quad\left(10,7\right.$, Incipit), Beginn durch Obelos bezeichnet, vergleiche $15^{\mathrm{L}}$ (7. Thut).

96 Eine Ausnahme sind Evangelistare wie $14^{\mathrm{L}}$ oder $297^{\mathrm{L}}$. Die Perikopenfolge der Messfeier (ordo major) ist: >(Psalm [Prokimenon] -) Pauline - Katholischer Brief - Acta - Psalm - Evangelium`, siehe Zanetti 1984: 6; Schüssler 2007: 319-321; Brakmann 2015. Liturgische Glossen, Titel und Vermerke werden in einem Katalog der Perikopenhandschriften und Lektionare im Sahidischen und in kleineren Literaturdialekten behandelt, siehe Schulz, Katalog. 
Punkte, vielleicht auch Angabe zweier Perikopenanfänge oder -enden.

L f. $4^{\mathrm{r}-\mathrm{v}}$ (Copte 129,10 f. 190; 18,1-6) r im Interkolumnium schrägstehendes Doppelkreuz vor 18,1, v Andreaskreuz bei 18,6 | (18,15, Incipit) v Andreas- und Kruckenkreuz teils übereinander geschrieben oberhalb der Initiale, wohl Markierung eines Perikopenbeginns, Schluss wegen Fragmentierung des Folgeblatts nicht auszumachen.

Nur 299 , Teil $\mathrm{D}^{\mathrm{v}}$ Zeile 4-19, bietet eine liturgische Rubrik, die auf das Johannesevangelium verweist, doch nicht den aufgenommenen Texten beigesellt werden kann, da sie nicht direkt vor oder am Text vorkommt. Wessely ${ }^{97}$ hat die Rubrik veröffentlicht. Editio princeps, Neuedition und Übersetzung seien nebeneinander gestellt, um Neulesungen und Ergänzungen besser zu erkennen:

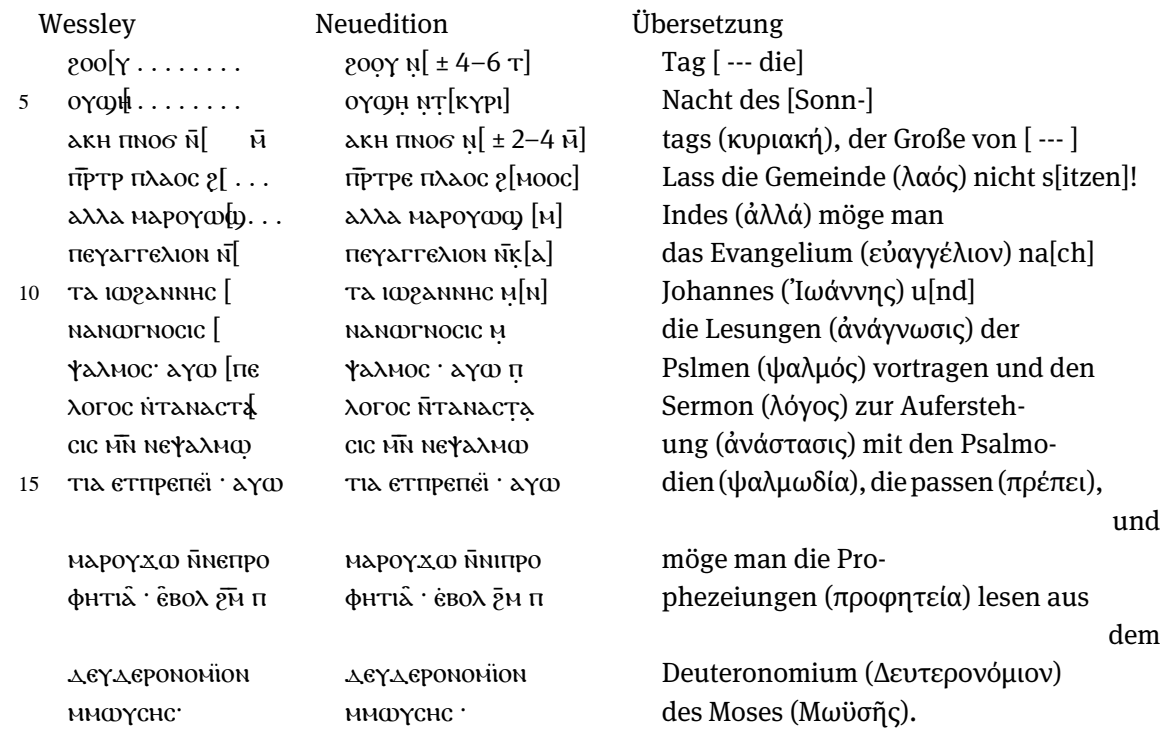

Die zunächst alphabetisch, sodann nach Handschriften gebotene Übersicht $\mathrm{zu}$ Überschriften und Glossen dokumentiert diejenigen Texte, die direkt vor oder am Johannesevangelium in den ausgewerteten Handschriften vorhanden sind..$^{98}$ In die tabellarische Übersicht zu Paratexten wurde zudem Material aus Volltexthandschriften einbezogen, das durch die Setzung von Initialen oder textkritischen Zeichen (interpretativ) Perikopenumfänge anzuzeigen vermag.

97 Edition: Wessely 1912: 194 Nr. 166b [Wien, ÖNB, K 9698].

$98 \mathrm{Zu}$ griechischen Texten siehe unter den Handschriftennummern die Listen von Schmitz/Mink und BC [zu 14 ${ }^{\mathrm{L}}$ siehe BC 3.4: 57, 59, 61-63 (dort statt Tremata Supralinearstriche bei Iota, also stets īrannнc statt ïгаnnнc, was nicht angeführt wird)]. 
Ein | ist Trennzeichen bei gleichem Text im selben Manuskript, || bei unterschiedlichen Handschriften mit identischem Text. Zwischen Manuskripten ist zur besseren Erkennbarkeit ein Spatium gesetzt. Angaben zur speziellen Erscheinung (Interpunktion, Verzierung, Diakritika) in Einzelhandschriften und generell, die im Haupteintrag unbenannt sind, sind in eckigen Klammern nachgesetzt. Defektive oder unsichere Lesungen, die wahrscheinlich identisch sind, sind in runden Klammern ergänzend einer Handschrift nachgestellt, ebenso mit zugehörigen bibliographischen Angaben abweichende Lesungen der Literatur. Als Kürzel für griechischen Text dient gr, und der Exponent ${ }^{\text {lac }}$ bezeichnet Textausfall am Beginn oder Ende von Perikopen.

unleserlich 103 T f. $2^{\text {r }}$ (Clarendon Press, b.2, Fragm. 8 f. 2; 19,33); Schmitz/Mink 1986: 78

unleserlich verblasst und Schrift der Gegenseite durchgeschlagen

$297^{\mathrm{L}} \mathrm{A}^{\mathrm{r}}(14,26-15,11)$; Schmitz/Mink 1991: 825

unleserlich verblasst und Schrift der Gegenseite durchgeschlagen $\quad 297^{\mathrm{L}} A^{\mathrm{v}}(15,26-16,2.4-9$. 13-15.19); Schmitz/Mink 1991: 825

$\overline{\mathrm{a}} \overline{\lambda \in z_{1} \mathrm{C}} \quad 396^{\mathrm{L}}$ p. $10(1,6,<3 .>$ Sonntag <des Hathor $>$, Zahlangabe von $\mathrm{m} 2)$

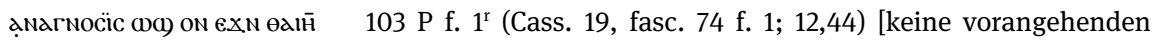

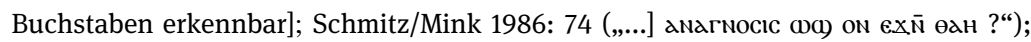

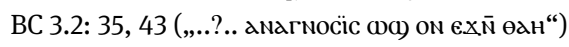

амre入oc 110 A f. $2^{\mathrm{v}}$ (P. 8775; 1,42); Schmitz/Mink 1986: 165; BC 3.3: 86-87

$\bar{\Gamma} \quad 116 \mathrm{~J}^{\mathrm{r}}(19,1<-12>$, Incipit, Karfreitag, 3. Tagesstunde); Schmitz/Mink 1986: 253; BC 3.2: 117,122

eк тоү ката ıюаnнn $\quad 335^{\mathrm{L}} \mathrm{C}^{\mathrm{v}}$ (20,24-31); Schmitz/Mink 1991: 980 (irrtümlich ıаannнn); BC 4.1: 44

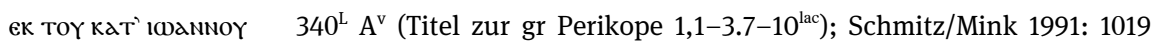

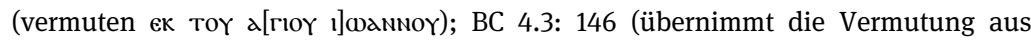
Schmitz/Mink 1991)

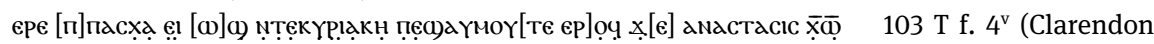
Press, b.2, Fragm. 8 f. 4; 20,24); Schmitz/Mink 1986: 78 (nur epe паı (0) ... und and-

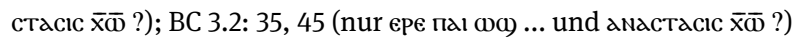

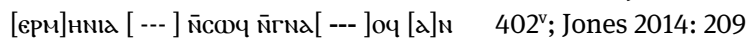

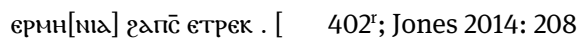

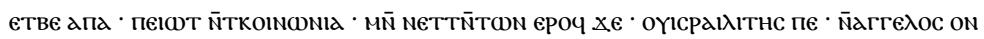

126 B f. $1^{\mathrm{r}}$

(Copte 129,9 f. 54; 1,43); Schmitz/Mink 1986: 409; BC 3.2: 50-51

етвє пюёік м̄пюме̄ $103 \mathrm{~K}^{\mathrm{v}}$ (6,35); Schmitz/Mink 1986: 69 (поєıк); ВС 3.2: 35, 41

eXN өגн 234v (wohl zu 7,37); Schmitz/Mink 1989: 611; BC 4.4: 112-113

eх⿱亠凶禸 оүархнепіскопос 103 O f. $2^{\mathrm{v}}$ (Copte 129,10 f. 133; 10,1); Schmitz/Mink 1986: 73; BC 3.2: 35, 43

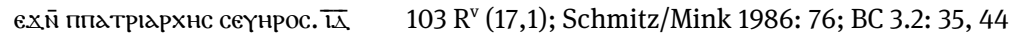

ExN T ... 103 O f. 3 v (Copte 129,10 f. 134; 10,22); Schmitz/Mink 1986: 73 („* Lesungshinweis zu 10,22?")

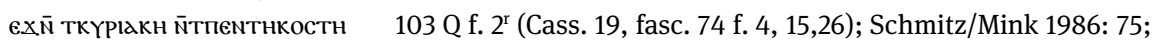
BC 3.2: 35, 44

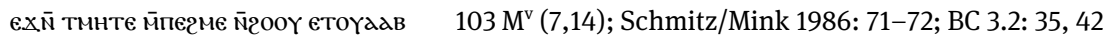


$116 \mathrm{~J}^{\mathrm{r}}$ (19,13<-27>, Karfreitag, 6. Tagesstunde); Schmitz/Mink 1986: 253; BC 3.2: 117, 122

$116 \mathrm{~J}^{\mathrm{v}}$ (19,28<-30>, Karfreitag, 9. Tagesstunde); Schmitz/Mink 1986: 254; BC 3.2: 117, 122

$\overline{\mathrm{Id}} \quad 116 \mathrm{~J}^{\mathrm{v}}$ (19,31, Karfreitag, 3. Tagesstunde); Schmitz/Mink 1986: 254; BC 3.2: 117, 122

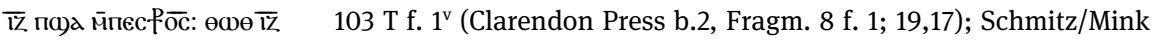

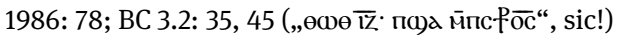

$\overline{\mathrm{ic}} \overline{\mathrm{xc}} \quad 408^{\mathrm{Lv}}$ (scriptio superior, links und rechts des oberen Teils des Kreuzpfahls)

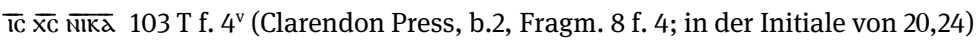

$\overline{1 \overline{2}} \quad 663^{\mathrm{L}}$ f. $39^{\mathrm{r}}$ (p. 67; 19,1, Karfreitag, 3. Tagesstunde, Incipit)

$\overline{1 \bar{\omega}} \overline{\mathrm{r}} \quad 396^{\mathrm{L}}$ p. $9(1,43,<2 .>$ Sonntag $<$ des Hathor $>)$

$\overline{\mathrm{i}} \overline{\mathrm{C}} \overline{\mathrm{lB}} \quad 396^{\mathrm{L}}$ p. $10(5,33,<3 .>$ Sonntag $<$ des Hathor $>)$

$\overline{\omega_{\overline{2}}} \cdot \overline{\mathrm{Ke}} \cdot 663^{\mathrm{L}}$ f. $49^{\mathrm{v}}$ (p. 98; 12,24, 4. Sonntag >zur Ernte<, Incipit)

$\overline{i \bar{\alpha}} \overline{\lambda \Lambda} \quad 663^{\mathrm{L}}$ f. $58^{\mathrm{r}}$ (p. <115>; 15,15, <4.> Sonntag <des Paone>, Incipit)

$\overline{1 \bar{\omega}} \overline{\mathrm{M \Lambda}} \cdot 663^{\mathrm{L}}$ f. $48^{\mathrm{r}}$ (p. $<95>; 20,19,<3$. Sonntag der Pentekoste>, Incipit)

· ката ıстаnnнс · $59^{\mathrm{Lr}}(16,20-23$, aus einem Sacramentale, Lesung für eine Frau, die im Kindbett verstarb)

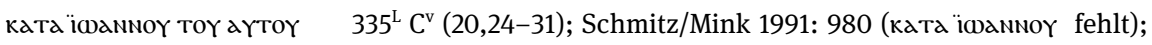
BC 4.1: 44

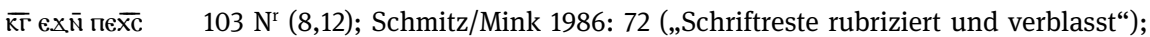
BC 3.2: 35,43

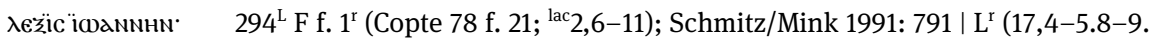

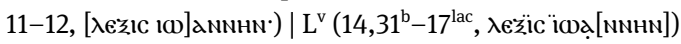

$\overline{\lambda \in \Xi I C} \overline{\kappa \Theta} \quad 396^{\mathrm{L}}$ p. $9(12,29,<2 .>$ Sonntag $<$ des Hathor $>)$

$\overline{\mathrm{ME}} \overline{\mathrm{i}} \overline{\mathrm{C}} \quad 663^{\mathrm{L}}$ f. $48^{\mathrm{v}}$ (p. 96; 21,12, 4. Sonntag der Pentekoste, Incipit, Abschnitts-Nr. von m2)

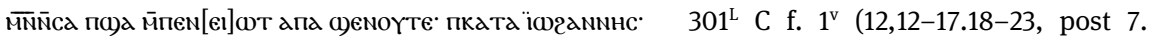
Epep); Schmitz/Mink 1991: 868 (

Pl $>\mathrm{d}>115 \mathrm{~K}^{\mathrm{v}}$ (siehe Anmerkung in der Handschriftenliste)

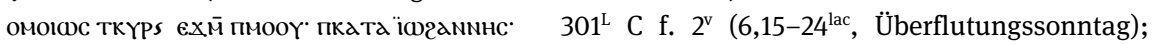
Schmitz/Mink 1991: 868-869

оүшг евод мпетма бюпє ом е̄техп்со 140 L f. $3^{\text {r }}$ (Or. 3579B(43) f. 70; 18,27, Desinit, und 18,28, Incipit; Kombination aus zwei Glossen: оүшг евод мпетмд und бюпе ом ётехпісо);

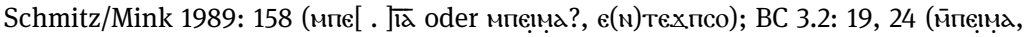
етехйсo)

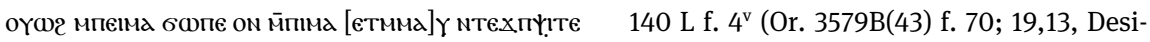
nit, und 19,14, Incipit; Kombination aus zwei Glossen: оүшг мпема und бюпе ом $\bar{M}-$ пимд [єтммд]Ү нтехпү!те); Schmitz/Mink 1989: 158 (,zu stark beschädigt, um gelesen

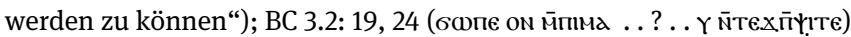

панто]к̣ратор $\rightarrow$ п паNто]к̣ратор паз пє печвшд $\quad 337^{\mathrm{L}} \mathrm{C}^{\mathrm{r}}\left(19,16-24^{\text {lac }}\right)$; Schmitz/Mink 1991: 998

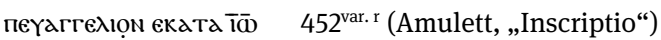

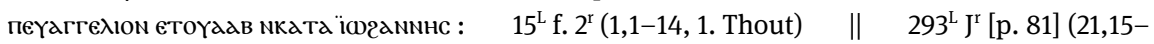

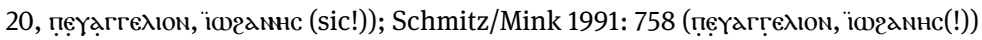

пеүагтедіon iorannhc $306^{\mathrm{L}}$ f. $4^{\mathrm{r}}$ (Copte 129,19 f. 72; 3,1-8 ${ }^{\text {lac }}$ ); Schmitz/Mink 1991: 903 (102anNHب̣); BC 4.1: 95

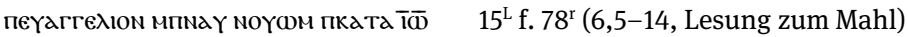

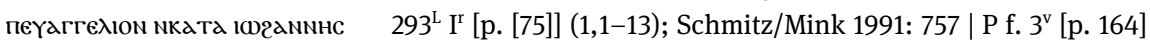




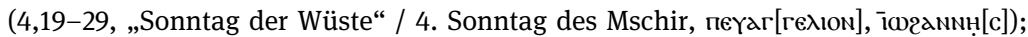

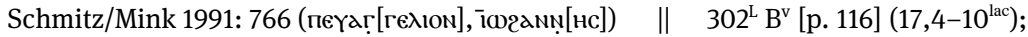
Schmitz/Mink 1991: 877 | Fv (7,37-45

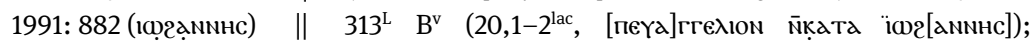

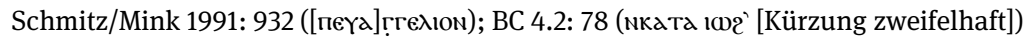
Varianten mit Zahlangabe:

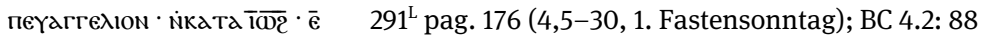

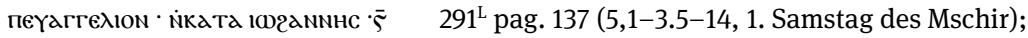
BC 4.2: 86 (玄, sic!)

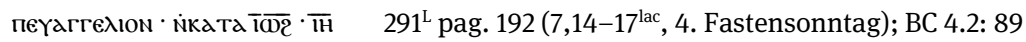

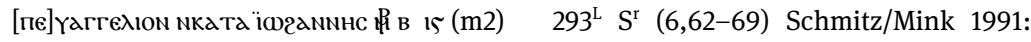
$770([\Pi]$ ẹarге入!on $)$

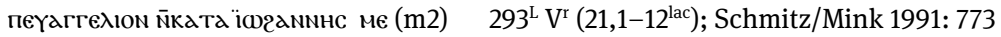

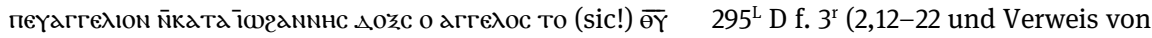

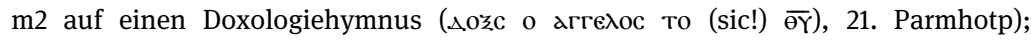

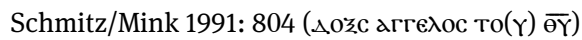

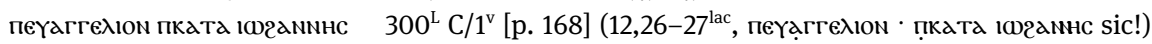
|| $324^{\text {Lr }}$ (11,5-9 [Textabbruch im Vers vor dem Kolumnenende]); Schmitz/ Mink 1991: 954 (ı⿻上丨nn.[HC]); BC 4.3: $169 \| \quad 440^{\mathrm{L}}$ f. $14^{\mathrm{r}}$ (f. $\left.\overline{\mathrm{KH}}^{\mathrm{r}}, 12,12-36\right)$

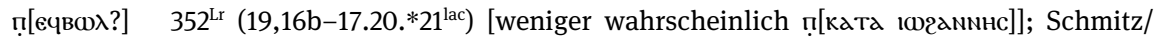
Mink 1989: 1064 (vermuten пєчвю入)

печвол $342^{\mathrm{L}} \mathrm{B}^{\mathrm{r}}(20,24-27.28-31)$; Schmitz/Mink 1991: 1032

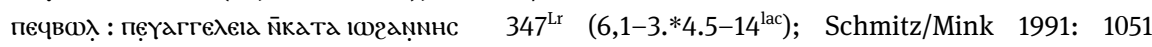

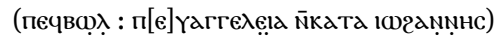

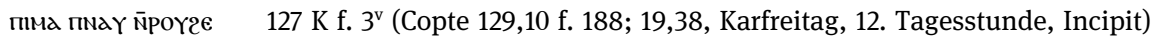
[N̄porę über пNaY]; Schmitz/Mink 1986: 427; BC 3.3: 70

пима п[наү м̄со] $127 \mathrm{~K} \mathrm{f.} 3^{\text {r }}$ (Copte 129,10 f. 188; 19,16, Karfreitag, 6. Tagesstunde, Incipit); Schmitz/Mink 1986: 427; BC 3.3: 70

пима пмаү м⿻)орт 127 K f. $1^{\text {v }}$ (Copte 129,10 f. 186; 18,28, Karfreitag, 1. Tagesstunde, Incipit);

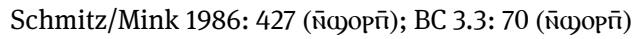

пимд 21 по)омте 127 K f. $2^{\text {r }}$ (Copte 129,10 f. 187; 19,1, Karfreitag, 3. Tagesstunde, Incipit); Schmitz/Mink 1986: 427; BC 3.3: 70

пимд хиүіте $127 \mathrm{~K} \mathrm{f.} 3^{\text {r }}$ (Copte 129,10 f. 188; 19,25, Karfreitag, 9. Tagesstunde, Incipit) [Folge tatsächlich хıүıте пıма]; Schmitz/Mink 1986: 427; BC 3.3: 70

пката "ісганnнс- [Interpunktion, Zierrat und Diakritika weichen in und zwischen Handschriften voneinander ab] $\quad 15^{\mathrm{L}}$ f. $5^{\mathrm{v}}(10,7-18,7$. Thout $) \mid$ f. $7^{\mathrm{r}}(12,20-32,15$. Thout $) \mid \mathrm{f}$. $9^{\mathrm{r}}(19,25-37,17$. Thout $) \mid$ f. $10^{\mathrm{v}}(15,7-17,9$. Hathor $) \mid$ f. $13^{\mathrm{r}}(1,43-51,13$. Hathor $) \mid$ f. $16^{\mathrm{r}}$ (17,1-11, 24. Hathor)| f. $31^{\mathrm{r}}\left(2,1-11,13\right.$. Tobe) | f. $56^{\mathrm{r}}\left(7,14-24\right.$, 4. Fastensonntag) | f. $58^{\mathrm{v}}$ $\left(3,1-15,6\right.$. Fastensonntag) | f. 59v (12,12-19, Palmsonntag)| f. 62 ${ }^{\mathrm{r}}$ (20,1-18, Ostersonntag)| f. $67^{\mathrm{v}}\left(20,19-31\right.$, Sonntag nach Ostern [Osteroktav]) | f. $73^{\mathrm{r}}(15,26-16,13$, Pfingsten) | f. 79 ${ }^{\mathrm{r}}$ (5,21-29, eine der Evangelienperikopen zum Begräbnis) [Zu Leseanlässen und Perikopenfolgen siehe BC 3.2: 62-70] $\| \quad 16^{\mathrm{L}}$ f. $5^{\mathrm{v}}$ (8,51-59, Montag der Pascha-Woche, 11. Tagesstunde) | f. $8^{\mathrm{r}}$ (12,35-43, Dienstag der Pascha-Woche, 3. Nachtstunde) | f. $18^{\mathrm{r}}$ (8,21-28, Dienstag der Pascha-Woche, 1. Tagesstunde) | f. 22 ${ }^{\mathrm{r}}(8,12-20$, Dienstag der Pascha-Woche, 6. Tagesstunde) |f. 46 ${ }^{\mathrm{v}}$ (11,55-57, Mittwoch der PaschaWoche, 11. Nachtstunde) | f. 51 ${ }^{\mathrm{r}}$ (11,46-53, Mittwoch der Pascha-Woche, 1. Tagesstunde) $\mid$ f. $53^{v}$ (12,1-8, Mittwoch der Pascha-Woche, 6. Tagesstunde) | f. 62 $(10,14-21$, 
Gründonnerstag, 1. Nachtstunde) | f. 65 (10,23-28, Gründonnerstag, 9. Nachtstunde) | f. $66^{\mathrm{r}}$ (10,29-42, Gründonnerstag, 11. Nachtstunde) | f. $71^{\mathrm{v}}$ (6,27-58, Gründonnerstag, 1. Tagesstunde) | f. $87^{\mathrm{v}}$ (13,1-20, Gründonnerstag, 9. Tagesstunde) |f. $97^{\mathrm{v}}(13,21-$ 30, Gründonnerstag, Synaxis) | f. 101 ${ }^{\mathrm{v}}$ (13,33-14,25, Karfreitag, 1. Nachtstunde) | f. $104^{\mathrm{v}}\left(14,26-15,25\right.$, Karfreitag, 1. Nachtstunde) | f. $107^{\mathrm{v}}$ (15,26-16,33, Karfreitag, 1. Nachtstunde) | f. $111^{\mathrm{r}}$ (17,1-26, Karfreitag, 1. Nachtstunde) | f. $115^{\mathrm{v}}$ (18,1-2, Karfreitag, 3. Nachtstunde) | f. $118^{\mathrm{v}}$ (18,3-9, Karfreitag, 6. Nachtstunde) | f. $122^{\mathrm{r}}(18,10-14$, Karfreitag, 9. Nachtstunde) | f. $127^{\mathrm{r}}\left(18,15-27\right.$, Karfreitag, 11. Nachtstunde) | f. $140^{\mathrm{v}}(18,28-$ 40, Karfreitag, 1. Tagesstunde) | f. $153^{\mathrm{v}}$ (19,1-12, Karfreitag, 3. Tagesstunde) | f. $165^{\mathrm{v}}$ (19,13-27, Karfreitag, 6. Tagesstunde) | f. 175 (19,28-30, Karfreitag, 9. Tagesstunde) | f. $181^{\mathrm{r}}$ (19,31-37, Karfreitag, 11. Tagesstunde) | f. $187^{\mathrm{r}}$ (19,38-42, Karfreitag, 12. Tagesstunde) [Zwischen пката und юәамnнс steht meist kein Punkt, sondern ein kreisförmiges bis ovales Trennzeichen, dass im Druck nicht nachahmbar ist und in Größe und Form einem Omikron ähnelt. Zu Leseanlässen und Perikopenfolgen siehe $\mathrm{BC}$ 1.4: 54-60.] \| 295 ${ }^{\mathrm{L}}$ B/1 f. $3^{\mathrm{r}}$ [CM 2630, G 529] (1,1-14, Weihnachten (29. Chojak), Orthinon) \| 298 C f. $2^{\mathrm{v}}$ [p. 130] (19,1- 16, Karfreitag, 3. Tagesstunde); Schmitz/Mink 1991: $840 \quad \| \quad 305^{\mathrm{L}} \mathrm{A}^{\mathrm{r}}$ (12,35-42); Schmitz/Mink 1991: 898; BC 4.3: $79 \mid \mathrm{B}^{\mathrm{r}}$ (7,37-46'ac , ?21. (oder 24.) Mesore, ıю2anN<H>c); Schmitz/Mink 1991: 899; BC 4.3: $82 \quad \| \quad 349^{\mathrm{L}}$ f. $2^{\mathrm{v}}$ (13,21-30); Schmitz/ Mink 1991: 1059

Variante mit Glosse von $\mathrm{m} 2$ :

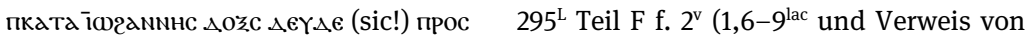
m2 auf einen Doxologiehymnus ( $\triangle 0 z \mathrm{c} \Delta \epsilon \curlyvee \Delta \epsilon$ (sic!) прос)); Schmitz/Mink 1991: 807 (iç2annhc)

пмаҮ мпалкт़̣р 127 K f. $1^{\text {r }}$ (Copte 129,10 f. 186; 18,10, Karfreitag, 9. Nachtstunde, Incipit);

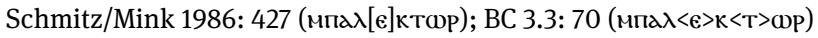

ппду г pbo p.75(Oberrand, Bedeutung unklar); Sharp 2016: (Spat. 23 ппдч| Spat. 24 г) псавватоп еХм̄ пмооҮ: - пката ̈̈ганnнс: - $\quad 301^{\mathrm{L}}$ C f. $2^{\mathrm{r}}$ (4,5.6-11.13-15, Überflutungssamstag); Schmitz/Mink 1991: 868

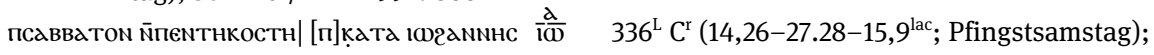

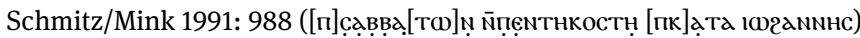

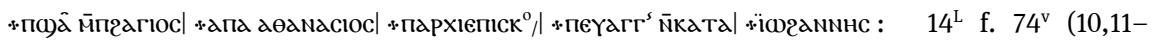
18, 7. Pachons); BC 3.4: 63

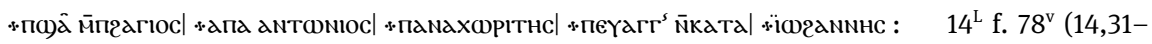
15,16, 21. Pachons); BC 3.4: 63

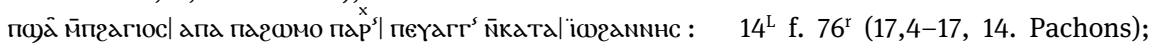
BC 3.4: 63

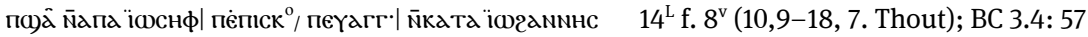

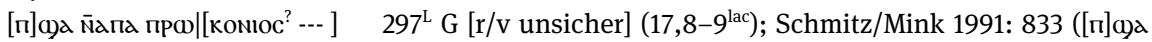
м̄апа прш[.? . ])

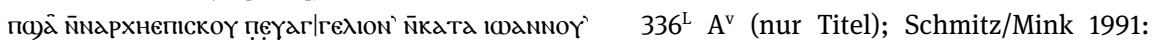

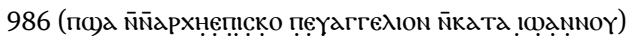

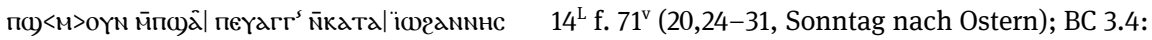
62

CION ח.10Y $154 \mathrm{E}^{\mathrm{r}}(13,23) ; \mathrm{BC} 3.4: 44$

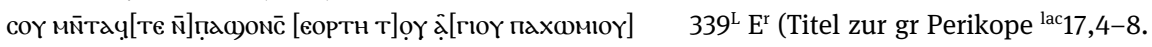

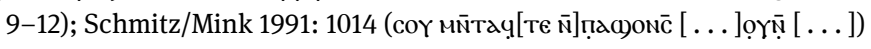

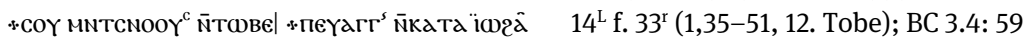




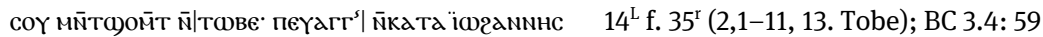

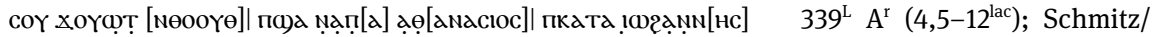

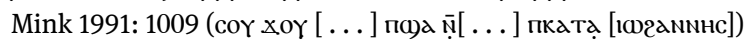

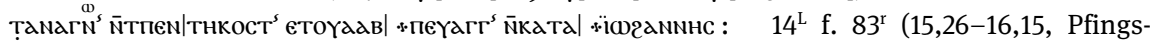
ten); BC 3.4: 63 (таNaгN" $\left.{ }^{\text {कs }}\right)$

ткүріакн $\bar{\lambda} \quad 154 \mathrm{~B}^{\mathrm{r}}(12,1)$; BC 3.4: 43

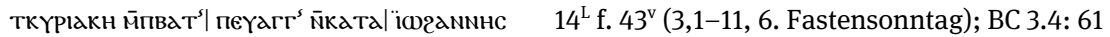

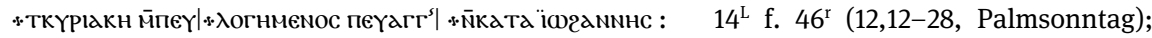
BC 3.4: 61

ткүріакн псіом 127 J f. $4^{v}$ (Ms. Or. 192(9) f. 17; 11,45-53, Palmsonntag [2 ×: am Außenrand ткүріакн мсіюм mit кн über pla in ткүріакн bei 11,45 und im Interkolumnium Reste von radiertem күріакн bei 11,53]); Schmitz/Mink 1986: 426 (,von anderer Hand eingetragen und im Interkolumnium getilgt“); BC 3.3: 69 (irrtümlich für 11,19 vermerkt)

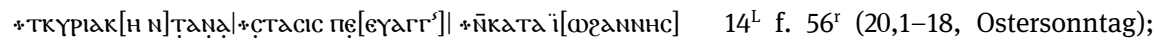
BC 3.4: 61 (ткүріak[H NT]аnactacic)

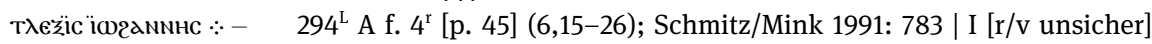

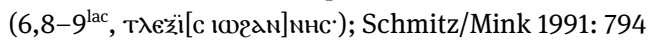

ToY aүṬ[or] $\quad$ Br (20,1.3-5.7-9.12-13.15-18 $\left.{ }^{\text {lac }}\right)$ Schmitz/Mink 1991: 980; BC 4.1: 43

тюве 110 A f. $2^{\mathrm{r}}$ (P. 8775; 1,39, <12.> Tobe); Schmitz/Mink 1986: 165; BC 3.3: 86-87

төве <е>твє пвд[п]тісма 110 A f. $1^{\text {r }}$ (Copte 129,9 f. 51; 1,29, <11.> Tobe); Schmitz/Mink 1986: 164 ([е]тве пвд[п]тісма); ВС 3.3: 86

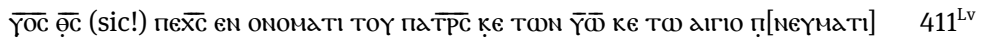

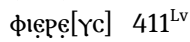

Ф паNто] ам.рттором...... [ 103 T f. $1^{\text {r }}$ (Clarendon Press, b.2, Fragm. 8 f. 1, 19,6); Schmitz/

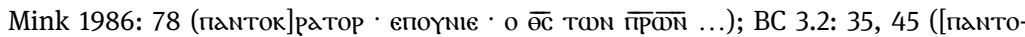

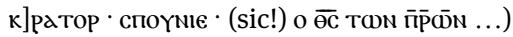

$\overline{\mathrm{j} \overline{\mathrm{d}}} \quad 663^{\mathrm{L}}$ f. $39^{\mathrm{r}}$ (p. 67; 19,16, Karfreitag, 3. Tagesstunde, Desinit)

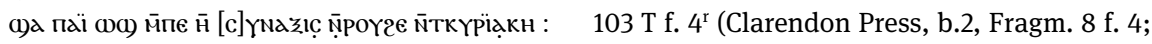

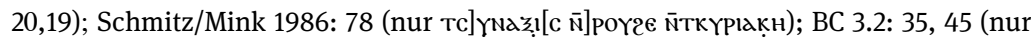

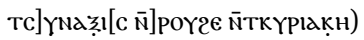

()а паї (о) мткүрідкн м̄пвод єво 103 T f. $3^{\text {r }}$ (Clarendon Press, b.2, Fragm. 8 f. 3; 20,1) ;

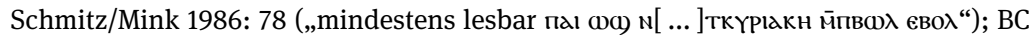
3.2: 35, 45 (паı (1)

c)д пाма No. $948 \mathrm{r}$ (wohl zu 19,41, Desinit)

бюпе он оүшг (евол) 


\section{A2.4.1. Nach Handschriften geordnete Übersicht zu Glossen, liturgischen Vermerken und Überschriften}

Der besseren Übersichtlichkeit wegen seien alle alphabetisch angeführten Angaben nach Handschriften geordnet wiederholt.

pbo ппас|г $\quad$ p. 75 (Oberrand, Bedeutung unklar); Sharp 2016: (Spat. 23 ппдч| Spat. 24 г)

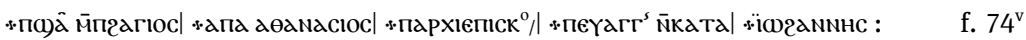
(10,11-18, 7. Pachons); BC 3.4: 63

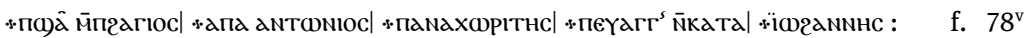
(14,31-15,16, 21. Pachons); BC 3.4: 63

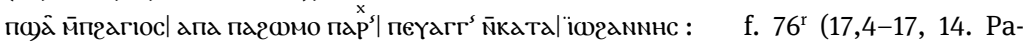
chons); BC 3.4: 63

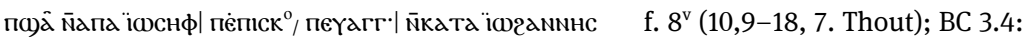
57

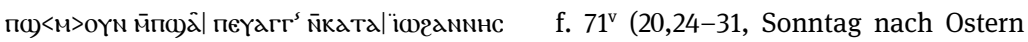
[Osteroktav]); BC 3.4: 62

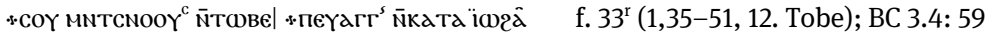

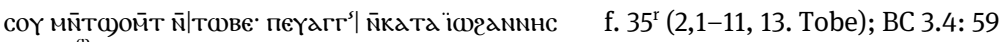

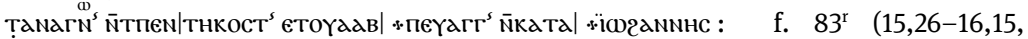
Pfingsten); BC 3.4: 63 (таNarN $\left.{ }^{\omega s}\right)$

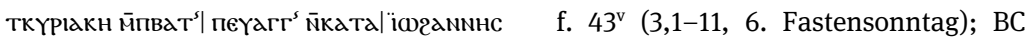
3.4: 61

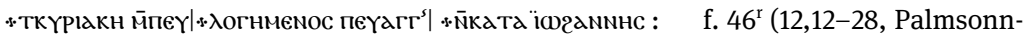
tag); BC 3.4: 61

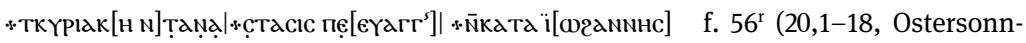

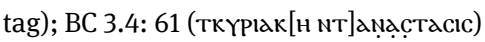
37, 17. Thout) | f. $10^{\mathrm{v}}\left(15,7-17,9\right.$. Hathor) | f. $13^{\mathrm{r}}\left(1,43-51,13\right.$. Hathor) | f. $16^{\mathrm{r}}(17,1-11$, 24. Hathor)| f. $31^{\mathrm{r}}\left(2,1-11,13\right.$. Tobe) | f. $56^{\mathrm{r}}$ (7,14-24, 4. Fastensonntag) | f. 58 $(3,1-15$,

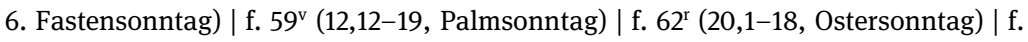
$67^{\mathrm{v}}$ (20,19-31, Sonntag nach Ostern [Osteroktav]) | f. 73 (15,26-16,13, Pfingsten) | f. $79^{\mathrm{r}}$ (5,21-29, eine der Perikopen zum Begräbnis) [Zu Leseanlässen und Perikopenfolgen siehe BC 3.2: 62-70] (12,35-43, Dienstag der Pascha-Woche, 3. Nachtstunde) | f. 18 ${ }^{\mathrm{r}}$ (8,21-28, Dienstag der Pascha-Woche, 1. Tagesstunde) | f. 22 ${ }^{\mathrm{r}}$ (8,12-20, Dienstag der Pascha-Woche, 6. Tagesstunde) | f. 46 (11,55-57, Mittwoch der Pascha-Woche, 11. Nachtstunde) | f. 51 ${ }^{\mathrm{r}}$ (11,46-53, Mittwoch der Pascha-Woche, 1. Tagesstunde) | f. 53v (12,1-8, Mittwoch der Pascha-Woche, 6. Tagesstunde) | f. 62 (10,14-21, Gründonnerstag, 1. Nachtstunde) | f. $65^{\mathrm{v}}$ (10,23-28, Gründonnerstag, 9. Nachtstunde) | f. 66 ${ }^{\mathrm{r}}$ (10,29-42, Gründonnerstag, 
11. Nachtstunde) | f. $71^{\mathrm{v}}$ (6,27-58, Gründonnerstag, 1. Tagesstunde) | f. $87^{\mathrm{v}}$ (13,1-20, Gründonnerstag, 9. Tagesstunde) | f. 97v (13,21-30, Gründonnerstag, Synaxis) | f. 101 (13,33-14,25, Karfreitag, 1. Nachtstunde) | f. $104^{\mathrm{v}}$ (14,26-15,25, Karfreitag, 1. Nachtstunde) | f. $107^{\mathrm{v}}$ (15,26-16,33, Karfreitag, 1. Nachtstunde) | f. $111^{\mathrm{r}}$ (17,1-26, Karfreitag, 1. Nachtstunde) | f. $115^{\mathrm{v}}$ (18,1-2, Karfreitag, 3. Nachtstunde) | f. $118^{\mathrm{v}}$ (18,3-9, Karfreitag, 6. Nachtstunde) | f. 122 $\left(18,10-14\right.$, Karfreitag, 9. Nachtstunde) | f. $127^{\mathrm{r}}(18,15-27$, Karfreitag, 11. Nachtstunde) | f. $140^{v}$ (18,28-40, Karfreitag, 1. Tagesstunde) | f. 153 (19,1-12, Karfreitag, 3. Tagesstunde) | f. 165 (19,13-27, Karfreitag, 6. Tagesstunde) | f. $175^{\mathrm{r}}$ (19,28-30, Karfreitag, 9. Tagesstunde) | f. 181 $1^{\mathrm{r}}$ (19,31-37, Karfreitag, 11. Tagesstunde) | f. $187^{\mathrm{r}}$ (19,38-42, Karfreitag, 12. Tagesstunde) [Zwischen пкатd und ıю2аNNHc steht meist kein Punkt, sondern ein kreisförmiges bis ovales Trennzeichen, das im Druck nicht nachahmbar ist und in Größe und Form einem Omikron ähnelt. $\mathrm{Zu}$ Leseanlässen und Perikopenfolgen siehe BC 1.4: 54-60.]

59 $\cdot$ катд ıюганnнс $\cdot \mathrm{r}(16,20-23$, aus einem Sacramentale, Lesung für eine Frau, die im Kindbett verstarb)

$\epsilon \mathrm{m} . \mid \mathrm{M}$. f. $2^{\mathrm{v}}$ (bei 14,2, Bedeutung unklar, letzter Buchstabe der 1. Zeile vielleicht $\Theta$, letzter Buchstabe der 2. Zeile vielleicht a oder $\lambda$ )

unleserlich $\quad$ T f. $2^{\text {r }}$ (Clarendon Press, b.2, Fragm. 8 f. 2; 19,33); Schmitz/Mink 1986: 78

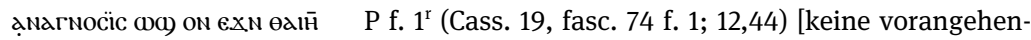

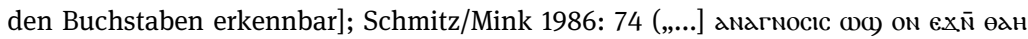

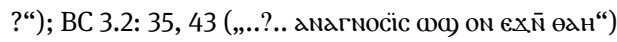

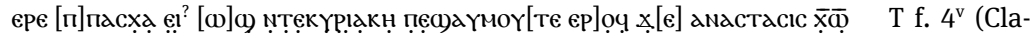
rendon Press, b.2, Fragm. 8 f. 4; 20,24); Schmitz/Mink 1986: 78 (lesen ерє паı (0)) ... und anactacic $\bar{x} \overline{\mathrm{D}}$ ?); BC 3.2: 35, 45 (übernimmt die Angabe aus Schmitz/Mink)

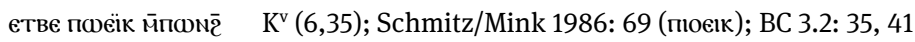

ex̄̄ оүархнепікопос O f. $2^{\mathrm{v}}$ (Copte 129,10 f. 133; 10,1); Schmitz/Mink 1986: 73; BC 3.2: 35,43

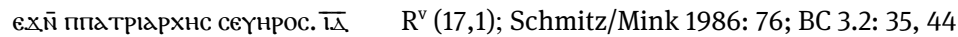
Ex. T .... O O f. $3^{\text {v }}$ (Copte 129,10 f. 134; 10,22); Schmitz/Mink 1986: 73 („* Lesungshinweis zu 10,22 ?“)

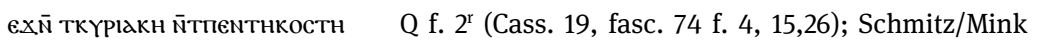
1986: 75; ВС 3.2: 35, 44

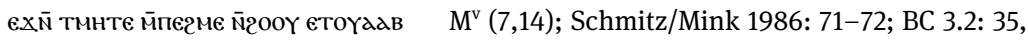
42

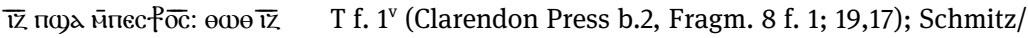

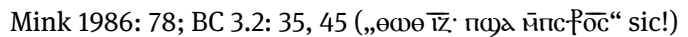

$\overline{\mathrm{IC}} \overline{\mathrm{xC}} \overline{\mathrm{NIKd}} \quad$ T f. $4^{\mathrm{v}}$ (Clarendon Press, b.2, Fragm. 8 f. 4; in der Initiale zu 20,24)

$\overline{\mathrm{K}} \mathrm{e} \overline{\mathrm{N}}$ пе $\overline{\mathrm{XC}} \quad \mathrm{N}^{\mathrm{r}}(8,12)$; Schmitz/Mink 1986: 72 (,Schriftreste rubriziert und verblasst“); BC 3.2: 35, 43

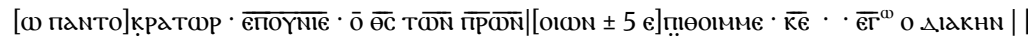
\pm 7 ] . амарттоо….... [ T f. $1^{\text {r }}$ (Clarendon Press, b.2, Fragm. 8 f. 1, 19,6);

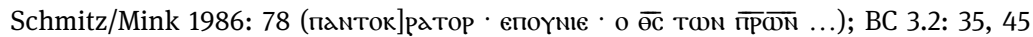

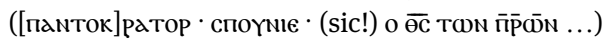

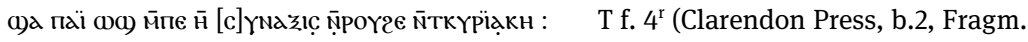




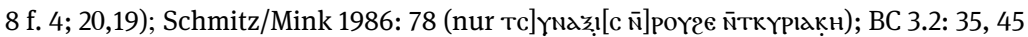

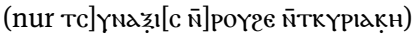

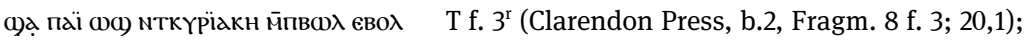

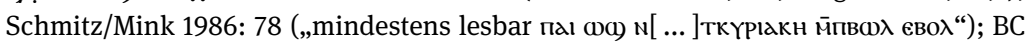

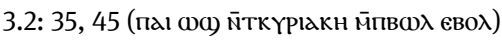

anredoc A f. 2v (P. 8775; 1,42); Schmitz/Mink 1986: 165; BC 3.3: 86-87 тове Af. $2^{\text {r }}$ (P. 8775; 1,39, <12.> Tobe); Schmitz/Mink 1986: 165; BC 3.3: 86-87 тове <е> тве пвд[п] тісма A f. $1^{\text {r }}$ (Copte 129,9 f. 51; (1,29, <11.> Tobe); Schmitz/ Mink 1986: 164 ([е]тве пвд[п]тісма); ВС 3.3: 86

$\mathbb{R}>\mathrm{d}>\quad \mathrm{K}^{\mathrm{v}}$ (siehe Anmerkung in der Handschriftenliste)

$\bar{r} \quad \mathrm{~J}^{\mathrm{r}}(19,1<-12>$, Karfreitag, 3. Tagesstunde); Schmitz/Mink 1986: 253; BC 3.2: 117, 122

$\bar{\varsigma} \quad J^{\mathrm{r}}(19,13<-27>$, Karfreitag, 6. Tagesstunde); Schmitz/Mink 1986: 253; BC 3.2: 117, 122

$\bar{\Theta} \quad J^{\mathrm{v}}(19,28<-30>$, Karfreitag, 9. Tagesstunde); Schmitz/Mink 1986: 254; BC 3.2: 117, 122

$\overline{\mathrm{Id}} \quad \mathrm{J}^{\mathrm{v}}$ (19,31, Karfreitag, 11. Tagesstunde); Schmitz/Mink 1986: 254; BC 3.2: 117, 122

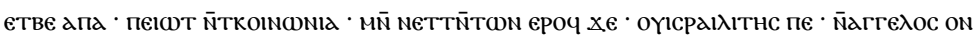
B f. $1^{\mathrm{r}}$ (Copte 129,9 f. 54; 1,43); Schmitz/Mink 1986: 409; BC 3.2: 50-51

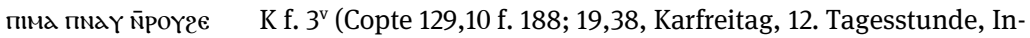

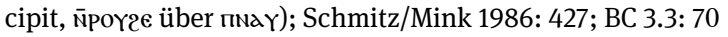

пाма п[Naץ $\overline{\mathrm{N}} \mathrm{co}] \quad$ K f. $3^{\mathrm{r}}$ (Copte 129,10 f. 188; 19,16, Karfreitag, 6. Tagesstunde, Incipit); Schmitz/Mink 1986: 427; BC 3.3: 70

пима пмаү ма)орп் K f. 1v (Copte 129,10 f. 186; 18,28, Karfreitag, 1. Tagesstunde, Inci-

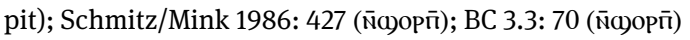

пима 21 паомте K f. $2^{\text {r }}$ (Copte 129,10 f. 187; 19,1, Karfreitag, 3. Tagesstunde, Incipit); Schmitz/Mink 1986: 427; BC 3.3: 70

пимд Хиүте $\quad$ K f. $3^{\text {r }}$ (Copte 129,10 f. 188; 19,25, Karfreitag, 9. Tagesstunde, Incipit, Folge tatsächlich хıүıте пाма); Schmitz/Mink 1986: 427; BC 3.3: 70

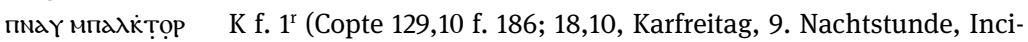

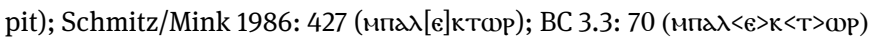

ткүріакн nсіюм J f. $4^{v}$ (Ms. Or. 192(9) f. 17; 11,45-53, Palmsonntag [2×: am Außenrand ткүріакн NсіळN mit кн über pla in ткүріакн bei 11,45 und im Interkolumnium Reste von radiertem күріакн bei 11,53]); Schmitz/Mink 1986: 426 (,von anderer Hand eingetragen und im Interkolumnium getilgt“); BC 3.3: 69 (irrtümlich für 11,19 vermerkt)

140 оүшг єво入 мпемма бюпє оп ётехпісо L f. $3^{\text {r }}$ (Or. 3579B(43) f. 70; 18,27, Desinit, und 18,28, Incipit; Kombination aus zwei Glossen: оүюг євод мпетмд und бюпє ом ёте-

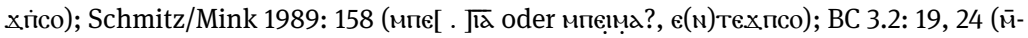
пемฺма, етехйсо)

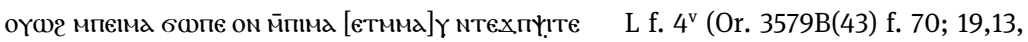
Desinit, und 19,14, Incipit; Kombination aus zwei Glossen: оүшг мпемма und бюпе ом мппима [єтмма]Ү мтехпү!те); Schmitz/Mink 1989: 158 (,zu stark beschädigt, um gele-

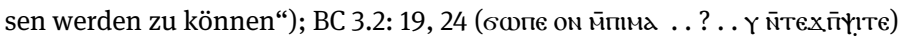


154

CiON ח.АIOY $\mathrm{E}^{\mathrm{r}}(13,23)$; BC 3.4: 44

ткүріакн $\bar{\Lambda} \quad \mathrm{B}^{\mathrm{r}}(12,1) ; \mathrm{BC} 3.4: 43$

EXN өגн $\quad \mathrm{v}$ (wohl zu 7,37); Schmitz/Mink 1989: 611; BC 4.4: 112-113

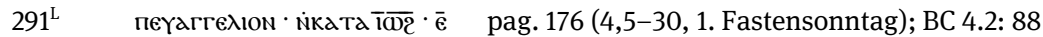

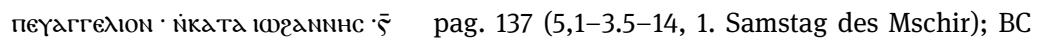
4.2: 86 (玄, sic!)

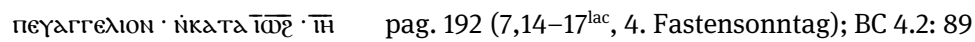

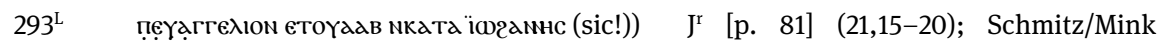
1991: 758 (п̣еүагтехіоn, їганнс(!))

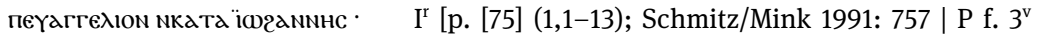
[p. 164] (4,19-29, ,Sonntag der Wüste“ / 4. Sonntag des Mschir, пеүaг[геגıon], ïran-

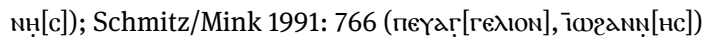

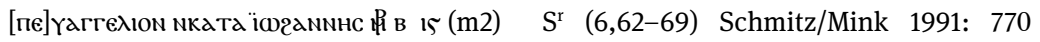

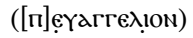

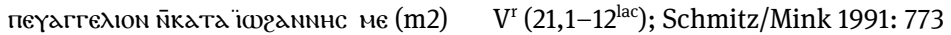

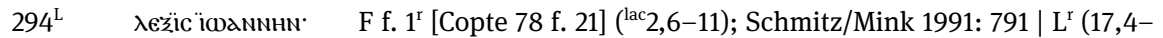

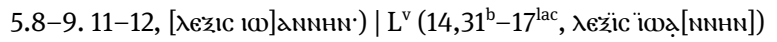

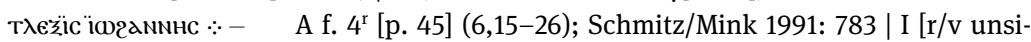

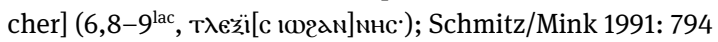

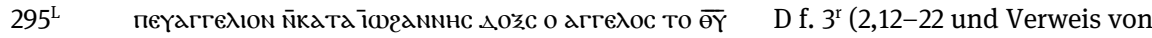

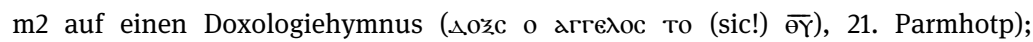

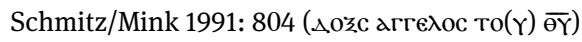

пката їганnнс $\quad$ B/1 f. $3^{\text {r }}$ (CM 2630, G 529; 1,1-14, 29.Chojak/Weihnachten, Orthinon)

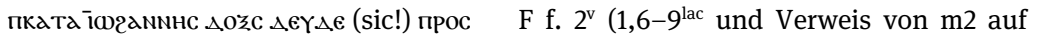
einen Doxologiehymnus ( $\triangle \mathrm{O} z \mathrm{C} \quad \Delta € \curlyvee \Delta \epsilon$ (sic!) прос)); Schmitz/Mink 1991: 807 (icugannнс)

$297^{\mathrm{L}} \quad$ unleserlich verblasst und Schrift der Gegenseite durchgeschlagen $\quad \mathrm{A}^{\mathrm{r}}(14,26-15,11)$; Schmitz/Mink 1991: 825

unleserlich verblasst und Schrift der Gegenseite durchgeschlagen $A^{v}$ (15,26-16,2.4-9. 13-15.19); Schmitz/Mink 1991: 825

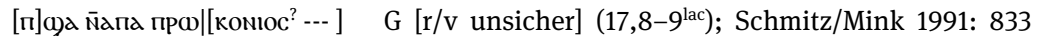

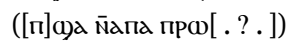

298 $\quad$ пката їогаnnнс- C f. $2^{\mathrm{v}}$ [p. 130] (19,1-16); Schmitz/Mink 1991: 840

300

301 7. Epep); Schmitz/Mink 1991: 868 (

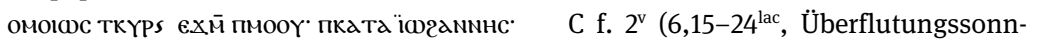
tag); Schmitz/Mink 1991: 868-869

псдввдтон ехм̄ пмооҮ: - пката їганnнс: - $\quad$ C f. $2^{\mathrm{r}}$ (4,5.6-11.13-15, Überflutungssamstag); Schmitz/Mink 1991: 868 


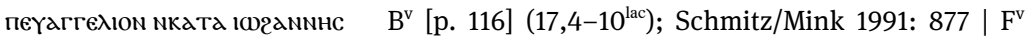

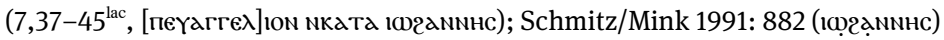

$305^{\mathrm{L}}$ пкатд ıюганnнс $\quad \mathrm{A}^{\mathrm{r}}(12,35-42)$; Schmitz/Mink 1991: 898; BC 4.3: 79| $\mathrm{B}^{\mathrm{r}}\left(7,37-46^{\text {lac }}\right.$, ?21. (oder 24.) Mesore, ıю2ann<H>c); Schmitz/Mink 1991: 899; BC 4.3: 82

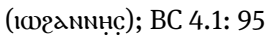

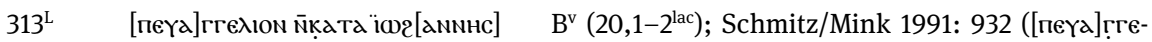

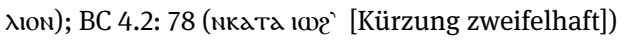

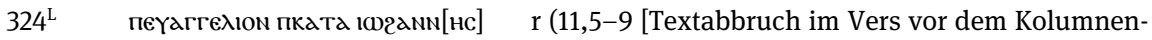
ende]); Schmitz/Mink 1991: 954 (ı̨̣ann][нс]); BC 4.3: 169

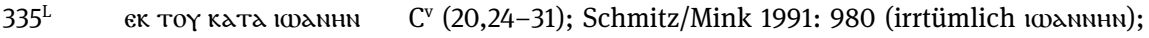
BC 4.1: 44

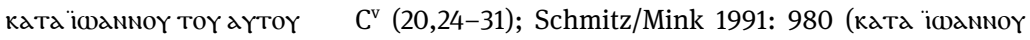
fehlt); BC 4.1: 44

ToY arț[oy] $\quad$ B ${ }^{\mathrm{r}}\left(20,1.3-5.7-9.12-13.15-18^{\text {lac }}\right)$ Schmitz/Mink 1991: 980; BC 4.1: 43

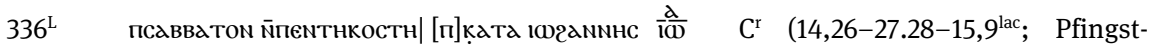

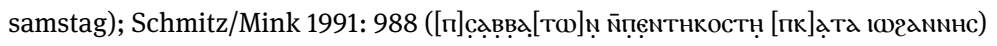

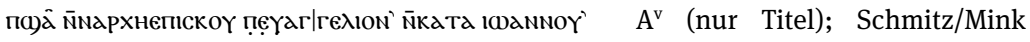

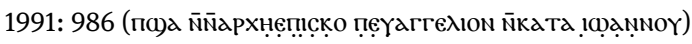

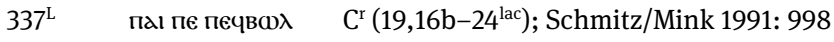

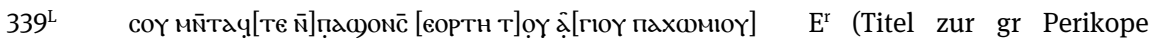

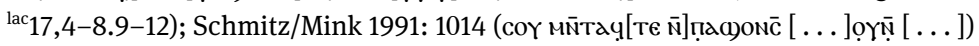

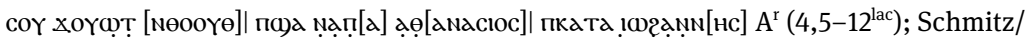
Mink 1991: 1009 (coY xoy [ . . ] по)а

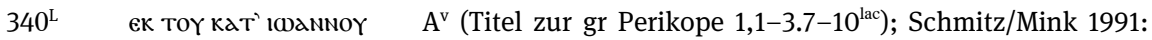

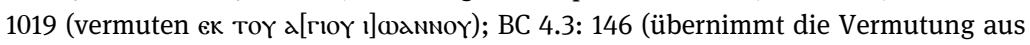
Schmitz/Mink 1991)

342

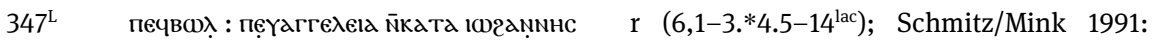

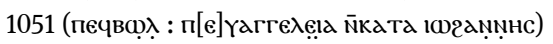

349 пката "ïrannhc f. $2^{\mathrm{v}}$ (13,21-30); Schmitz/Mink 1991: 1059

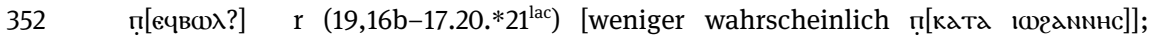
Schmitz/Mink 1989: 1064 (vermuten пєчвш入)

$396^{\mathrm{L}} \overline{\mathrm{a}} \overline{\lambda \epsilon \xi_{1 \mathrm{C}}} \quad$ p. $10(1,6,<3 .>$ Sonntag $<$ des Hathor $>$, Zahlangabe von m2)

$\overline{\mathrm{i} \overline{\mathrm{C}} \overline{\mathrm{r}}} \overline{\mathrm{r}} \quad$ p. $9(1,43,<2 .>$ Sonntag $<$ des Hathor $>)$

$\overline{\mathrm{i}} \overline{\mathrm{lB}} \quad$ p. $10(5,33,<3 .>$ Sonntag $<$ des Hathor $>)$

$\overline{\lambda \in Z_{I C}} \overline{\mathrm{K} \theta} \quad$ p. $9(12,29,<2 .>$ Sonntag $<$ des Hathor $>)$

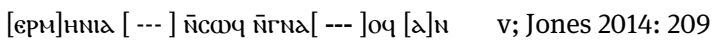




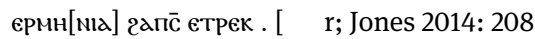

408 $\quad \overline{\mathrm{L}} \overline{\mathrm{xC}} \quad \mathrm{v}$ (scriptio superior, links und rechts des oberen Teils des Kreuzpfahls)

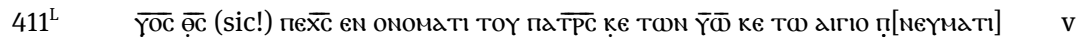
фıере [үс] v

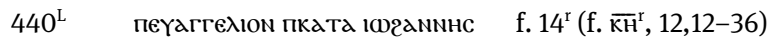

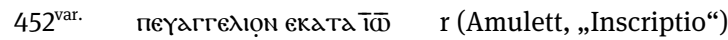

$663^{\mathrm{L}} \quad \overline{\bar{\omega}} \overline{2} \quad$ f. $39^{\mathrm{r}}$ (p. 67; 19,1, Karfreitag, 3. Tagesstunde, Incipit)

$\overline{\bar{\omega}} \cdot \overline{\mathrm{ke}} \cdot \quad$ f. $49^{\mathrm{v}}$ (p. 98; 12,24, 4. Sonntag >zur Erntes, Incipit)

$\overline{i \omega 2} \overline{\lambda \Lambda} \quad$ f. $58^{\mathrm{r}}$ (p. <115>; 15,15, <4.> Sonntag <des Paone>, Incipit)

$\overline{102} \overline{M \Lambda} \cdot \quad$ f. $48^{\text {r }}$ (p. $<95>; 20,19,<3$. Sonntag der Pentekoste $>$ Incipit)

$\overline{\mathrm{ME}} \overline{\mathrm{i}} \overline{\mathrm{O}}$ f. $48^{\mathrm{v}}$ (p. $96 ; 21,12,4$. Sonntag der Pentekoste, Incipit, Abschnitts-Nr. von

$\mathrm{m} 2)$

$\overline{a \bar{\lambda}} \quad$ f. $39^{\mathrm{r}}$ (p. $67 ; 19,16$, Karfreitag, 3. Tagesstunde, Desinit)

No. 948 क)а пाма $\quad \mathrm{r}$ (wohl zu 19,41, Desinit) 


\section{A2.5. Methoden der Textstrukturierung}

Der Text des Johannesevangeliums ist in den ausgewerteten Handschriften unterschiedlich strukturiert. Teils ist eine Textaufteilung durch die Setzung von Initialen integraler Bestandteil der Manuskriptanlage, teils sind unterschiedliche Nummerierungssysteme als gliederndes Element beigefügt, die nur selten ursprünglich, sondern meist zu einem späteren Zeitpunkt von einer zweiten oder auch dritten bzw. rezenten Hand gesetzt wurden. ${ }^{99}$ Textkritische Zeichen (Paragraphoi, Diplen, Obeloi oder Koroniden) werden ebenfalls zur Strukturierung verwandt. ${ }^{100}$ Gewöhnlich werden verschiedene Methoden kombiniert, etwa Initialen mit textkritischen Zeichen oder Initialen mit textkritischen Zeichen und Nummerierungen.

Nummerierungen zur Strukturierung des Textes lassen sich grob in drei Kategorien einteilen:

a.) Kephalaia nach griechischem Modus,

b.) Kephalaia nach koptischem Modus, zur besseren Unterscheidung nachfolgend als Abschnittsnummern bezeichnet, und

c.) Eusebianische Kanonzahlen.

Ein spezielles, sonst nicht belegtes System der nummerischen Textstrukturierung hat 4 . Von 1,51 bis 21,18 werden 25 Passagen mit гамнм гамнм (Amen-Worte Jesu) von $\bar{\alpha}-\overline{\kappa \epsilon}$ durchgezählt. ${ }^{101}$ Die Schrift von Text und Nummerierungen ist sehr ähnlich und stammt recht sicher von derselben Person. Der Grund der Nummerierung ist nicht zu ermitteln, doch scheint es denkbar, dass die Textauswahl zu Studienzwecken vorgenommen und/oder der Kodex für exegetische Arbeiten herangezogen wurde.

Bei Angaben und Auflistungen weitgehend außen vor bleiben als genuin zum liturgischen Gebrauch geschaffene Manuskripte Lektionare und Perikopenhandschriften; sie werden nur dann angeführt, wenn sie durch Kephalaia- oder Abschnittsnummern Angaben liefern, die neutral in eine deskriptive Beschrei-

99 Die einzige Handschrift, bei der die Gestaltung durch den Kopisten bereits auf die Zufügung mehrerer Systeme der Texteinteilung anhand nummerierter Gliederungsabschnitte hin konzipiert worden zu sein scheint, ist 131. Hier sind Eusebianische Kanonzahlen und, teils mit Titeln, Kephalaia nach griechischem Modus in verkleinerter, feiner Schrift korrespondierend zu Initialsetzungen und Paragraphoi dem Text beigefügt.

100 Paragraphoi können zudem Kopisten als Hilfsmittel dienen, wie sich für pbo nachweisen lässt, da hier nahezu jede Seite mit einem Paragraphos am Beginn des Schriftspiegels versehen ist. Hier sind Paragraphoi schlicht ein Mittel, das dem Kopisten einen Anhaltspunkt lieferte, an welcher Stelle des Papyrus der Schriftspiegel ansetzen sollte.

101 Schmitz/Mink 1986: 9; BC 3.1: 37. 
bung der Strukturierungsmethoden aufgenommen werden können. Dies gilt für $291^{\mathrm{L}}, 292^{\mathrm{L}}$ und $293^{\mathrm{L}}$ - teils sind bei Lesungen Nummern gesetzt, die sich nicht auf ein einzelnes Nummerierungssystem beziehen lassen bzw. teils fehlerhaft zu sein scheinen. Als Zahlen werden in $291^{\mathrm{L}}$ genannt: $\overline{\mathrm{e}}(4,5), \bar{\varsigma}(5,1)$ und $\overline{\mathrm{IH}}(7,14)$. Für die ersten beiden Zahlen lässt sich recht sicher annehmen, dass es sich um Kephalaia nach griechischem Modus handelt, auch wenn $\bar{\epsilon}$ um eine Stelle verzählt sein dürfte. $\overline{\mathrm{IH}}$ hingegen lässt sich den Abschnittsnummern (Kephalaia nach koptischem Modus) zuweisen. $292^{\mathrm{L}}$ bietet drei Male $\overline{\lambda z}(18,1,18,10$ und 18,15), ferner $\overline{\lambda \Theta}(19,1) .{ }^{102}$ Es sind zweifellos Abschnittsnummern, doch sollte die dreifache Nennung von $\overline{\lambda z}$ als Verweis auf den gesamten Abschnitt angesehen werden und nicht als feinere Unterscheidung oder anderweitige Gliederung. In

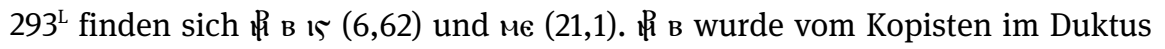
der Überschrift und mit derselben Tinte geschrieben, doch die anderen Zahlangaben stammen von anderer, sehr ungelenker Hand. is $(6,62)$ und me $(21,1)$ lassen sich als Abschnittsnummern interpretieren, auch wenn is $(6,62)$ recht genau zwischen die sonst überlieferten Anfänge der Abschnitte $\overline{15}(6,47)$ und $\bar{\gtrless}(6,70)$ fällt. Es dürfte der Perikopenumfang (6,62-69) als Ausschnitt des Abschnitts vermerkt worden sein. mє $(21,1)$ hingegen entspricht genau der Position anderer Handschriften. Für die Angabe der Martyrie ( sich keine sichere Erklärung bieten, doch scheint ein Verweis auf ein Nummerierungssystem vorzuliegen, dessen Bedeutung gegenwärtig unbekannt ist. Für das Johannesevangelium ist nur in 115 ebenfalls als Glosse eine Martyrie verzeichnet ( $P_{1}$ a bei 10,22), die interpretativ mit einer Perikopenliste in 264 in Verbindung gebracht werden kann. ${ }^{103}$ Mit aller Vorsicht lässt sich ein speziell liturgisches nummerisches Verweissystem vermuten, für das zu wenige Belege im Johannesevangelium vorhanden sind, weshalb andere biblische Texte zur Verifizierung oder Falsifizierung überprüft werden müssen.

Nur erwähnt seien einige Zahlangaben in 10, 109 und 285: Bei 285 sind von einer rezenten Hand außer der ursprünglichen Nummerierung mit Kanon-, Kephalaion- und Abschnittsnummern am Oberrand der Rectoseiten Kephalaionnummern gesetzt, die den Seitentext angeben sollen. Da sich die Zahlen nicht auf Anfangsverse beziehen, Zählfehler vorliegen und sie kein Beleg für die koptische Texteinteilung sind, werden sie nur in der Überblickstabelle mit relativer Position angeführt, doch nicht beim Abschnitt zu Kephalaia nach griechischem Modus. Ebenso wird mit Kephalaianummern verfahren, die in 10 von einer re-

102 Die Angabe „<38>: 18,28-40“ in BC 2.2: 67 ist nur emendiert und zu tilgen.

103 Es handelt sich um einen Verweis auf 10,22, in 264 genannt als Lesung für den Sonntag

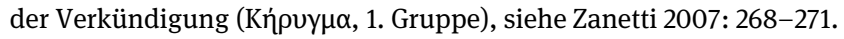


zenten Hand hinzugefügt wurden. ${ }^{104}$ In 109 sind Abschnittsnummern von zweiter Hand gesetzt, doch auch am Oberrand einiger Seiten von einer dritten Hand. Auf Teil $M^{r}$ findet sich oberhalb der inneren Kolumne $(8,54-9,2)$ die Zahl $\overline{\kappa r}$. Da 9,1 mit $\overline{\mathrm{KB}}$ bezeichnet ist, ist $\overline{\mathrm{K}}$ hier recht sicher fehlerhaft gesetzt und wird nicht aufgenommen.

Die Setzung von Initialen und textkritischen Zeichen (Paragraphoi, Diplen, Obeloi, Koroniden), fast immer im Verbund, ist die verbreitetste Methode der Textstrukturierung. Häufig sind Paragraphoi und andere textkritische Zeichen Initialen lediglich als Zierelemente beigesellt, sodass ihre Setzung oft nicht von der der Initialen interpretativ zu scheiden ist. In einigen Manuskripten besitzen sie indes unzweifelhaft eigenständig eine textstrukturierende Funktion (so etwa in pbo, 1, 3, 126, 162 oder 181).

Die Setzung von Initialen in Lektionaren und Perikopenhandschriften wird nicht verzeichnet; sie sollte nicht mit derjenigen in Volltexthandschriften zusammengeführt werden, da so verschiedene Intentionen der Textstrukturierung vermengt und letztlich nivelliert werden, denn zum liturgischen Gebrauch produzierte Handschriften sind per se als Lesemanuskripte konzipiert. Ihre Texteinteilung durch Initialen kann und wird vielfach mit Volltexthandschriften zusammengehen, doch unterliegen liturgische Handschriften anderen Verwendungsgesetzmäßigkeiten als Manuskripte, die zuvörderst der Textvervielfältigung dienen. Gleichwohl lässt sich bereits für die als älteste Manuskripte angesehenen Volltexthandschriften eine Wechselwirkung zur liturgischen Verwendung des Textes feststellen. ${ }^{105}$

104 Sie scheinen mit Bleistift geschrieben worden zu sein und sind in Hyvernats Faksimileedition nicht zu sehen, siehe Hyvernat 1922, obwohl sie am Original deutlich erkennbar sind. Es kann als sicher gelten, dass sie erst nach 1922 angebracht wurden.

105 Siehe Schulz 2021. 


\section{A2.5.1. Kephalaia (griechischer Modus)}

Obgleich Kephalaia nach griechischem Modus als nummerisches Strukturierungssystem zum Johannesevangelium über die koptische Texttradition hinaus verbreitet sind, sind sie nur in einer geringen Anzahl sahidischer Manuskripte nachweisbar. Allein 9 überliefert eine vollständige Liste der zugehörigen Titel, die nicht mit den wenigen als Glossen vermerkten Angaben anderer Handschriften gänzlich übereinstimmt. Aufgrund der spärlichen Überlieferung sind die Angaben aus 9 Referenzpunkt zur Verwendung von Kephalaia nach griechischen Modus, dürfen indes aber nicht verabsolutiert werden. Die Liste, die bereits von $\mathrm{H}$. Quecke ${ }^{106}$ ediert wurde, sei als Neuedition vorgelegt.

Von den 18 Nummern der Kephalaia-Liste aus 9 waren Kephalaion $\overline{\mathrm{H}}$ und $\overline{\mathrm{z}}$ nicht am Text an Abbildungen auszumachen, entweder weil der Schreiber die Nummern ausgelassen hat oder weil sie auf vorliegenden, eigentlich sehr guten Bildern unkenntlich sind. In der Kephalaion-Liste lauten die Einträge $\overline{\mathbf{H}}$ єтвє өє

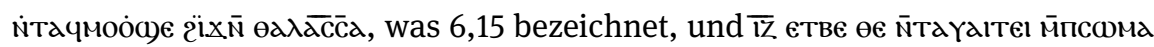
$\overline{\mathrm{NIC}}$, was auf 19,38 verweist.

Die Kephalaia-Liste spiegelt nicht exakt die Einteilung in griechischer Tradition wider. ${ }^{107}$ Der 4. Titel entspricht nicht dem 4. Kephalaion griechischer Einteilung $(3,25)$, das ungenannt ist, sondern deren 5. Kephalaion (4,5). Hernach geht die griechische Zählung somit um je eine Nummer voraus. Der 17. Titel „Über die Grablegung “ $(19,38)$ entspricht dem 18. und abschließenden Kephalaion der meisten griechischen Handschriften. Der 18. Titel in 9, „Über die Auferstehung“, wird nur in wenigen griechischen Handschriften als 19. Kephalaion genannt. Zur einfacheren Übersicht sind neben die Edition die Kephalaion-Nummern in Ziffern mit den zugehörigen Textstellen wiederholt.

Unterschiede von Supralinearpunkten $\mathrm{zu}$-strichen zwischen Queckes und dieser Edition sind als geringe Abweichung unverzeichnet, fehlende Diakritika werden vermerkt.

106 Edition: Quecke 1984: 223.

107 Siehe für die griechische Einteilung von Soden 1911: 411. Der Text der sahidischen Kephalaion-Titel ist nahezu identisch mit den bei von Soden angegebenen griechischen Texten. 
New York, PM, M 569 f. $84^{\mathrm{v}}$

1

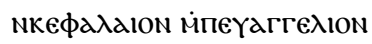

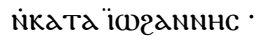

a

1. 2,1

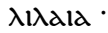

5 B

2. 2,12

гм' прпє

г етве NIко $\triangle$ нмос

3. 3,1

스 етве тесёіме N̄camaрітнс

4. 4,1

є етве пвасілікос .

5. 4,46

6. 5,1

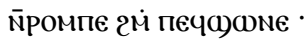

z

7. 6,1 Bं cNaY.

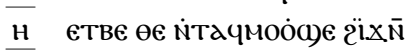

8. 6,16

15

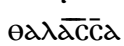

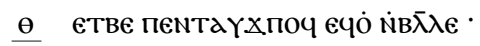

9. 9,1

1 eтве $\lambda$ dZAPOC.

10. 11,1

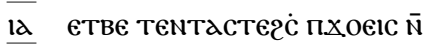

11. 12,1

COGN

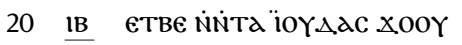

12. 12,4

Iг Єтвє пею்

13. 12,12

스 eтве NoYéeineIn ÑTaYt

14. 12,20 пеүоүӧ ёроч .

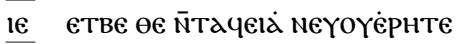

15. 13,1

16. 14,26

25 is етве ппарак入нтос ·

17. 19,38

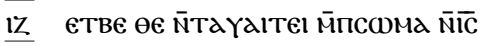

18. 20,1

iH etbe tanactacic.

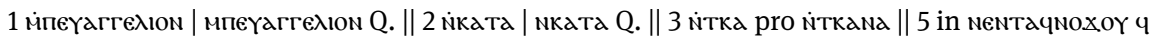

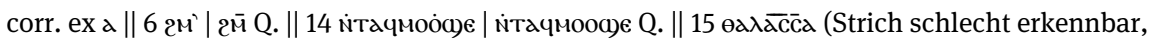
wohl durchgezogen über das Wortende hinaus) | eadacca Q. || 16 Überstreichung nach links hin stark verlängert || 24 меүоүёрнте | меүоүєрнте Q. 
Liste der Kephalaia nach griechischem Modus ${ }^{108}$

$\bar{\lambda} \quad 2,1 \quad 9$ f. $86^{\mathrm{v}}$ (pag. $\bar{\Delta}$ ); Schmitz/Mink 1986: $22 \|$ || 47 f. $4^{\mathrm{v}}$ (pag. $\overline{\mathrm{H}}, \mathrm{m} 2$ ) [Nummer fehlt]; BC 3.4: 33$\}$

$\overline{\mathrm{B}} \quad 2,12 \quad 9$ f. $87^{\mathrm{r}}$ (pag. $\bar{e}$ ); Schmitz/Mink 1986: $22 \|\left\{47\right.$ f. $5^{\mathrm{r}}$ (pag. $\bar{e}, \mathrm{~m} 2$ ) [Nummer fehlt]; BC 3.4: 33 (irrtümlich für 2,13)\}

$\overline{\mathbf{r}} \quad 3,1 \quad 9$ f. $87^{\mathrm{r}}$ (pag. ē); Schmitz/Mink 1986: 22

$\bar{\Lambda} \quad 4,1 \quad 9$ f. $88^{\mathrm{v}}$ (pag. $\overline{\text { H}}$ ); Schmitz/Mink 1986: 22

$\bar{\epsilon} \quad 4,46 \quad 9$ f. $90^{\mathrm{r}}$ (pag. $\overline{\mathrm{ld}}$ ); Schmitz/Mink 1986: 22

4,5 291 pag. $\overline{\text { Por }}$ (m2, wohl Zählfehler); BC 4.2: 88

$\bar{\varsigma} \quad 5,1 \quad 9$ f. $90^{\mathrm{r}}$ (pag. $\overline{\mathrm{l}}$ ); Schmitz/Mink 1986: 22|| $291^{\mathrm{L}}$ pag. $\overline{\mathrm{P} \lambda \overline{\mathrm{Z}}}(\mathrm{m} 2) ; \mathrm{BC} 4.2: 86$ (irrtümlich $\bar{\xi}$ )

$\overline{\mathrm{z}} \quad 6,1 \quad 9$ f. $91^{\mathrm{v}}$ (pag. $\overline{1 \perp}$ ); Schmitz/Mink 1986: 22

$\overline{\mathrm{H}} \quad 6,169$ f. $92^{\mathrm{r}}$ (pag. $\bar{\imath}$ ) [kein Verweis am Text, nur Keph.-Titel]; Schmitz/Mink 1986: 22 (mit Fragezeichen für 6,70)

$\bar{\theta} \quad 9,1 \quad 9$ f. $97^{\mathrm{v}}$ (pag. $\overline{\mathrm{kS}}$ ); Schmitz/Mink 1986: 22

i $\quad 9,1 \quad 285$ C f. $3^{\text {r }}$ (Hunt. 4 f. 7) [*]; BC 3.4: 75, 76 (für 8,59)

11,1 9 f. $100^{\mathrm{r}}$ (pag. $\overline{\lambda \bar{d}}$ ); Schmitz/Mink 1986: 22

$\overline{1 d} \quad 11,1 \quad 142 \mathrm{D}^{\mathrm{v}}$; Schmitz/Mink 1989: 181; BC 3.4: $17 \| 197 \mathrm{~B}^{\mathrm{v}}$ (cass. 18 fasc. 68 f. 2) [Titel unter der Kol.]; Schmitz/Mink 1991: 511; BC 4.3: 159, 160 || 285 C f. $15^{\mathrm{r}}$ (Hunt. 4 f. 18) [* und rezent]; BC 3.4: 75, 76

12,1 9 f. $101^{\mathrm{v}}$ (pag. $\overline{\lambda \triangle}$ ); Schmitz/Mink 1986: 22

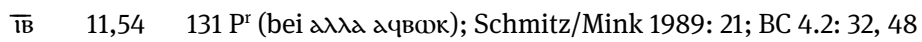

12,4 9 f. $101^{\mathrm{v}}$ (pag. $\overline{\lambda \Delta}$ ); Schmitz/Mink 1986: 22

$\bar{\pi} \quad$ 12,4 $131 \mathrm{P}^{\mathrm{v}}$; Schmitz/Mink 1989: 21 (fälschlich zu 12,3); BC 4.2: 32, 48

12,12 9f. 102 ${ }^{\mathrm{r}}$ (pag. $\overline{\lambda \epsilon}$ ); Schmitz/Mink 1986: 22

$\overline{1} \quad 12,12 \quad\left\{131 \mathrm{Q}^{\mathrm{r}}\right.$ [Textverweis und Text nicht, aber Titel erhalten]; Schmitz/Mink 1989: 22; BC 4.2: 31, 48 (fälschlich zu 12,14)\} || 132 || 285 D f. $1^{\mathrm{r}}$ (Hunt. 4 f. 22) [*]; BC 3.4: 75, 76

12,20 9 f. $102^{\mathrm{r}}$ (pag. $\overline{\lambda \epsilon}$ ); Schmitz/Mink 1986: 22

$\overline{i \in} \quad 12,20 \quad\left\{131 \mathrm{Q}^{\mathrm{r}}\right.$ [Verweis und Text nicht, aber Titel erhalten]; Schmitz/Mink 1989: 22 [Titel nicht gelesen]; BC 4.2: 31, 48 [Titel nicht gelesen, verweist

108 Bei 285 sind neben ursprünglichen Kanon-, Kephalaion- und Abschnitts-Nrn. von rezenter Hand am Oberrand aller Rectoseiten griechische Kephalaion-Zahlen gesetzt; die Zahlen beziehen sich nicht auf Anfangsverse und Zählfehler liegen vor. Sie sind kein Beleg für die Einteilung in koptischer Tradition und werden nicht angeführt. Ebenso bleiben von rezenter Hand bei 10 zugefügte Kephalaia unerwähnt. Sie scheinen mit Bleistift geschrieben worden zu sein und sind in Hyvernats Faksimileedition nicht erkennbar, siehe Hyvernat 1922, obwohl sie am Original deutlich sichtbar sind. Sicher wurden sie erst nach 1922 angebracht. 
auf 9, doch fälschlich auf 12,21]\} || 285 D f. $2^{\text {r }}$ (Hunt. 4 f. 23) [*]; BC 3.4:

75,76

13,1 9 f. $103^{\mathrm{r}}$ (pag. $\left.\overline{\lambda z}\right)$; Schmitz/Mink 1986: 22

$\overline{15} \quad 14,26 \quad 9$ f. $105^{v}$ (pag. $\overline{\mathrm{MB}}$ ); Schmitz/Mink 1986: 22

$\left\{13,4 \quad\right.$ BC 3.4: 108, 113 nennt $132 \mathrm{M}^{\mathrm{v}}$ bei 13,4 für Keph. $\overline{15}$, doch handelt es sich um eine Fehlinterpretation durchgeschlagener Tinte\}

\ $\quad$ 15,26 124 L f. 2v (m2, Copte 129,10 f. 169); BC 3.2: 126, 131

19,38 9 f. $111^{\mathrm{r}}$ (pag. $\overline{\mathrm{N}}$ ) [kein Verweis am Text, nur Keph.-Titel]; Schmitz/Mink 1986: 22 (,,ausgelassen (?)“)

$\overline{\mathrm{IH}} \quad 20,1 \quad 9$ f. $111^{\mathrm{v}}$ (pag. $\overline{\mathrm{N} \triangle}$ ); Schmitz/Mink 1986: 22

In Handschriften überlieferte Kephalaion-Titel nach griechischem Modus

\begin{tabular}{|c|c|c|c|}
\hline 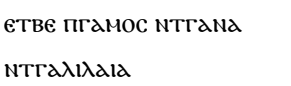 & $\{\bar{\alpha}\}$ & 2,1 & 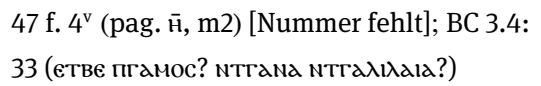 \\
\hline 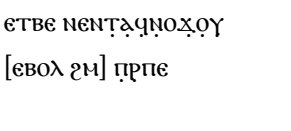 & $\{\overline{\mathrm{B}}\}$ & 2,12 & $\begin{array}{l}47 \text { f. } 5^{\mathrm{r}} \text { (pag. } \bar{\theta}, \mathrm{m} 2 \text { ) [Nummer fehlt]; BC 3.4: } \\
33 \text { („єтвє ... von 2. Hand?“, ohne Verszuwei- } \\
\text { sung) }\end{array}$ \\
\hline єтвє $\lambda$ дсарос & $\overline{1 d}$ & 11,1 & $\begin{array}{l}197 B^{v} \text { (cass. } 18 \text { fasc. } 68 \text { f. 2) [Titel unter der } \\
\text { Kol.]; Schmitz/Mink 1991: 511; BC 4.3: 159, } \\
160\end{array}$ \\
\hline 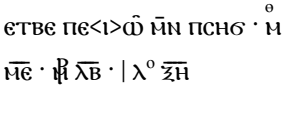 & $\overline{\Lambda \Lambda}$ & 12,12 & 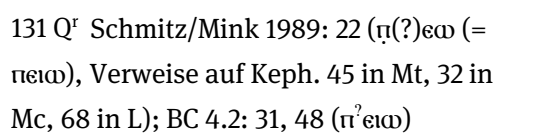 \\
\hline 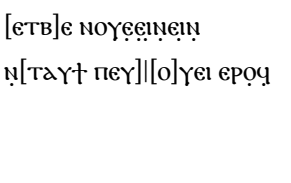 & $\overline{1 e}$ & 12,20 & $\begin{array}{l}131 \mathrm{Q}^{\mathrm{r}} \text { [Verweis am Text und Text nicht er- } \\
\text { halten]; Schmitz/Mink 1989: } 22 \text { [Titel nicht } \\
\text { gelesen]; BC 4.2: 31, } 48 \text { [Titel nicht gelesen, } \\
\text { verweist auf 9] }\end{array}$ \\
\hline єтвє ппарак入нтос & $\bar{z}$ & 15,26 & $\begin{array}{l}124 \text { L f. } 2^{v} \text { (Copte 129,10 f. 169; zugleich Titel } \\
\text { der koptischen Abschnitts-Nr.?); BC 3.2: 126, } \\
131\end{array}$ \\
\hline
\end{tabular}




\section{A2.5.2. Abschnittsnummern (Kephalaia nach koptischem Modus)}

Die nummerische Texteinteilung, die am ehesten „koptisch“ genannt werden könnte und in der bohairischen Texttradition recht verbreitet ist, sind Abschnittsnummern bzw. Kephalaia nach koptischem Modus. Sahidische und bohairische Abschnittsnummern korrelieren beim Johannesevangelium nur teilweise. ${ }^{109}$ Erstaunlicherweise sind fast keine Titel zu den sahidischen Kephalaia überliefert, und die Mehrzahl der Titel, die sämtlich von zweiter Hand stammen, sind erkennbar verkürzte Angaben.

Liste der Abschnittsnummern bzw. Kephalaia nach koptischem Modus

$\bar{d} \quad 1,6 \quad 134 \mathrm{O}^{\mathrm{r}}$; Schmitz/Mink 1989: 74; BC 4.1: 32 || 396 p. 10 (m2, Angabe zum Perikopenincipit)

$\overline{\mathrm{B}} \quad 1,18 \quad 134 \mathrm{O}^{\mathrm{v}}$

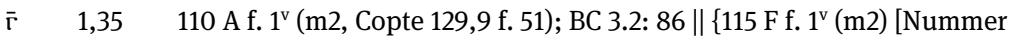
fehlt tatsächlich]; Schmitz/Mink 1986: 232; BC 3.2: 105, 109\} || 116 E f. $1^{v}$ (Copte 129,9 f. 33); Schmitz/Mink 1986: 248; BC 3.2: 120

$1,43 \quad 396^{\mathrm{L}}$ p. 9 (Angabe zum Perikopenincipit)

$\bar{\Lambda} \quad 2,1 \quad 110$ A f. $2^{\mathrm{v}}$ (m2, P. 8775); BC 3.2: 86-87 || 115 F f. $2^{\mathrm{r}}$ (m2); Schmitz/Mink 1986: 232; BC 3.2: 105, 109 ( $<\bar{\Delta}>$, Nummer emendiert, doch nur verwischt) || 116 E f. $2^{\mathrm{r}}$ (Copte 129,9 f. 34); BC 3.2: 120 || 167 A f. $1^{\mathrm{r}}$ (Copte 129,9 f. 53); Schmitz/Mink 1989: 387, 388; BC 3.4: 38 || 181 C f. $4^{\text {r }}$; von Lemm 1885: 19; Schmitz/Mink 1989: 455 (Angabe nach von Lemm) 110 A f. $3^{\text {r }}$ (Copte 129,9 f. 52); BC 3.2: 86-87 || 115 F f. $2^{v}$ (m2); Schmitz/ Mink 1986: 232; BC 3.2: 105, 109 || 116 E f. $2^{v}$ (Copte 129,9 f. 34); BC 3.2: 120 || 167 A f. $1^{\text {v }}$ (Copte 129,9 f. 53); Schmitz/Mink 1989: 388; BC 3.4: 38 || 181 C f. $4^{\text {r}}$; von Lemm 1885: 19; Schmitz/Mink 1989: 455 (Angabe nach von Lemm)

$\bar{r} \quad 3,1 \quad 10$ pag. $\overline{\mathrm{H}}\left(\overline{\mathrm{s}}, \mathrm{m} 2, \mathrm{CM} 3820\right.$ (481), G 938) \|| 115 F f. $3^{\mathrm{r}}$ (m2); Schmitz/Mink 1986: 232; BC 3.2: 105, 109 || 116 E f. $3^{\text {r }}$ (Copte 129,9 f. 35); BC 3.2: 120 || 167 A f. $2^{\text {r }}$ (Copte 129,9 f. 68-70); Schmitz/Mink 1989: 388; BC 3.4: 38

$\bar{z} \quad 3,22 \quad 115$ F f. $3^{\mathrm{v}}$ (m2); Schmitz/Mink 1986: 232; BC 3.2: 105, $109(<\bar{z}>)$ [Nummer emendiert, doch nur verwischt] || 116 E f. $4^{\mathrm{r}}$ (Copte 129,9 f. 36); BC 3.2: 120 || 167 A f. $2^{\text {v }}$ (Copte 129,9 f. 68-70); Schmitz/Mink 1989: 388; BC 3.4: 38 || 181 C f. $5^{\text {r }}$; von Lemm 1885: 21; Schmitz/Mink 1989: 455 (Angabe nach von Lemm)

109 Für Titel und Versangaben in bohairischer Überlieferung siehe Hebbelynck 1928: 81-120. 
110 B; BC 3.2: 86-87 || 115 F f. $5^{\mathrm{r}}$ (m2); Schmitz/Mink 1986: 232; BC 3.2: 105, 109 || 116 E f. $5^{\mathrm{r}}$ (Copte 129,9 f. 37); BC 3.2: 120 || $139 \mathrm{C}^{\mathrm{v}}$ (m2); Schmitz/Mink 1989: 133; BC 3.3: 41 || 167 A f. $3^{\text {r }}$ (Copte 129,9 f. $71+133,2$ f. 92a); Schmitz/Mink 1989: 387, 388; BC 3.4: 39 || 181 C f. $5^{\mathrm{v}}$; von Lemm 1885: 22; Schmitz/Mink 1989: 455 (Angabe nach von Lemm) 10 pag. $\overline{1 \xi}\left(\mathrm{m} 2, \mathrm{CM} 3820\right.$ (481), G 927) || 116 E f. $6^{\mathrm{v}}$ (Copte 129,9 f. 38); BC 3.2: 120 12 (Or. 14149(20) Fragm. $1^{\mathrm{r}}$ ) 116 E f. $7^{\text {r }}$ (Copte 129,9 f. 39); BC 3.2: 120 *10 pag. $\overline{\mathrm{IH}}\left(\overline{1 \mathrm{a}}, \mathrm{m} 2\right.$, CM 3820 (504), G 910) || 116 E f. $7^{\mathrm{v}}$ (Copte 129,9 f. 39); BC 3.2: 120 12 (Or. 14149(23) Fragm. 1v); BC 3.1: 65 (Fehlangaben) 10 pag. $\overline{\text { Kd }}$ (m2, CM 3820 (504), G 910) || 116 E f. $8^{\text {v }}$ (Copte 129,9 f. 40); BC 3.2: 120 || 167 B $^{\mathrm{r}}$; Schmitz/Mink 1989: 387, 390; BC 3.4: 40 $396^{\mathrm{L}}$ p. 10 (Angabe zum Perikopenincipit)

6,1 116 E f. $9^{\text {r }}$ (Copte 129,9 f. 41); BC 3.2: 120 || 139 D (m2); Schmitz/Mink 1989: 134; BC 3.3: 42 || 167 B ; Schmitz/Mink 1989: 387, 390; BC 3.4: 40 10 pag. $\overline{\kappa . \Lambda}$ (m2, CM 3820 (523), G 918); Schmitz/Mink 1986: 27 || *116 E f. $9^{v}$ (Copte 129,9 f. 41); BC 3.2: 120

6,24 10 pag. $\overline{\mathrm{ke}}$ (m2, CM 3820 (508), G 943) || 116 E f. $10^{\mathrm{r}}$ (Copte 129,9 f. 42); BC 3.2: 120

6,47 10 pag. $\overline{\text { KF }}$ (m2, CM 3820 (505), G 906) || 116 E f. $11^{\text {r }}$ (Copte 129,9 f. 43); BC 3.2: 120

6,62 $293^{\mathrm{L}} \mathrm{S}^{\mathrm{r}}(\mathrm{m} 2$, Angabe am Perikopenbeginn, aber wohl auf 6,47-69 insgesamt zu beziehen); Schmitz/Mink 1991: 770

6,70 10 pag. $\bar{\lambda}$ (m2, CM 3820 (512), G 912) || 116 E f. $12^{\text {r }}$ (Copte 129,9 f. 44); BC 3.2: 120 || $139 \mathrm{~F}^{\mathrm{r}}(\mathrm{m} 2)$; Schmitz/Mink 1989: 136; BC 3.3: 42

7,14 10 pag. $\overline{\lambda d}$ (m2, CM 3820 (513), G 911, $\overline{1 \mathrm{lt}}$ ) || $116 \mathrm{E}$ f. 12 (Copte 129,9 f. 44); BC 3.2: 120 || 162 C f. $1^{\mathrm{v}}$ (Copte 61 f. 1); BC 3.4: 99 || $285 \mathrm{~A}^{\mathrm{r}}$ (Hunt. 4 f. 1); BC 3.4: $75 \|$ || 291 ${ }^{\mathrm{L}}$ pag. $\overline{\mathrm{PQB}}(\mathrm{m} 2)$; BC 4.2: 89

7,37 10 pag. $\overline{\lambda \triangle}$ (m2, CM 3820 (518), G 894) \| \{115 Gv (m2) [Zahl tatsächlich nicht vorhanden]; Schmitz/Mink 1986: 233; BC 3.2: 105, 110 (fälschlich e))\} || 116 E f. $13^{\text {r }}$ (Copte 129,9 f. 45); Schmitz/Mink 1986: 248; BC 3.2: 120 ||

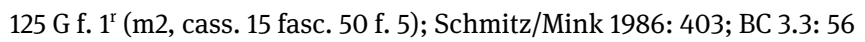
|| 162 C f. 2v (Copte 61 f. 2); BC 3.4: 99 || 250r; Schmitz/Mink 1991: 649 10 pag. $\overline{\lambda \epsilon}$ (m2, CM 3820 (519), G 914) || 116 F f. $1^{\mathrm{r}}$ (Or. 3579B(36) f. 1); Schmitz/Mink 1986: 249; BC 3.2: 120 || 125 G f. $1^{\mathrm{v}}$ (m2, cass. 15 fasc. $50 \mathrm{f}$. 5); Schmitz/Mink 1986: 403; BC 3.3: 56 || $139 \mathrm{G}^{\mathrm{r}}$ (m2); Schmitz/Mink 1989: 137; BC 3.3: 43

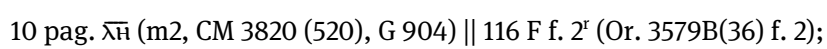


Schmitz/Mink 1986: 249; BC 3.2: 120 || 125 G f. $2^{\mathrm{r}}$ (m2, cass. 15 fasc. 50 f. 6); Schmitz/Mink 1986: 403; BC 3.3: 56 (irrtümlich für 8,31) \| 155 B ; Schmitz/Mink 1989: 318; BC 3.4: 67 || 285 B f. $2^{\mathrm{v}}$ (Hunt. 4 f. 3); BC 3.4: 75

8,31 $115 \mathrm{H}^{\mathrm{r}}$ (m2); Schmitz/Mink 1986: 234; BC 3.2: 105, 110

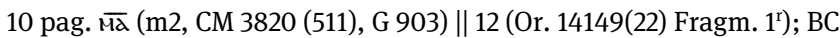
3.1: 65 (Fehlangaben) || 109 Mv'; Schmitz/Mink 1986: 161 („Kephalaionzählung [...] unklar und wohl teilweise zerstört“, indes erhalten) || $125 \mathrm{G}$ f. 3v (m2, cass. 15 fasc. 50 f. 7); Schmitz/Mink 1986: 403; BC 3.3: 56 || 285 C f. $4^{\mathrm{r}}$ (Hunt. 4 f. 7); BC 3.4: 74, 76 (nennt fälschlich $\overline{\mathrm{HB}} / \mathrm{l}$ (sic!) für 8,59)

10,1 neren Kol.); Schmitz/Mink 1986: 161 || $174 \mathrm{~B}^{\mathrm{v}}$ (m2) BC 4.3: 70 || 285 C f. $9^{\mathrm{v}}$ (Hunt. 4 f. 12); BC 3.4: 75, 76

$\overline{\mathrm{K} \Delta} \quad 10,22$ 10 pag. $\overline{M H}$ (m2, CM 3820 (524), G 920); Schmitz/Mink 1986: $27 \| 115 \mathrm{~J}^{\mathrm{v}}$ ( $\mathrm{m} 2$, von dritter Hand in Form der Paginierung $\cdot \uparrow_{\mathrm{l}}>\mathrm{d}>$ [Martyrie d, siehe Anhang 2 - 1.5.]); Schmitz/Mink 1991: 1144; BC 3.2: 105, 111 (Vermerk dritter Hand unerwähnt) || 285 C f. 12v (Hunt. 4 f. 15); BC 3.4: 75, 76 236; BC 3.2: 111 || 285 C f. $15^{\mathrm{r}}$ (Hunt. 4 f. 18); BC 3.4: 75, 76 $134 \mathrm{R}^{\mathrm{r}}$ (m2); Schmitz/Mink 1989: 139; BC 3.3: 43 156 || 116 Gv (m2); Schmitz/Mink 1986: 250; BC 3.2: 121 || $134 \mathrm{R}^{\mathrm{v}}$ || $139 \mathrm{I}^{\mathrm{v}}$ BC 3.2: 121

$12,1 \quad 12\left(\mathrm{~m} 2\right.$, Or. $14149(22)$ Fragm. $\left.1^{\mathrm{r}}, \overline{\mathrm{KH}}\right)$

12,12 285 D f. $1^{\text {r }}$ (Hunt. 4 f. 22); BC 3.4: 75, 76

*10 pag. $\overline{\mathrm{NH}}$ (m2, fast unleserlich, CM 3820 (495), G 913) || $116 \mathrm{G} / 1^{\mathrm{v}}$ (m2); BC 3.2: 121 || 285 D f. $2^{\text {r }}$ (Hunt. 4 f. 23); BC 3.4: 75, 76

12,24 $663^{\mathrm{L}}$ f. $49^{\mathrm{v}}$ (p. 98; Angabe zum Perikopenincipit)

12,29 396 $\mathrm{L}$ p. 9 (Angabe zum Perikopenincipit)

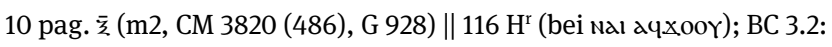
121 || 139 J (m2, bei nal ayxooץ); Schmitz/Mink 1989: 140; BC 3.3: 44 10 pag. $\overline{\mathrm{ZB}_{\mathrm{B}}}\left(\mathrm{m} 2\right.$, CM 3820 (480), G 905) || $116 \mathrm{H}^{\mathrm{v}}$; BC 3.2: 121 || $132 \mathrm{M}^{\mathrm{r}}$; BC 3.4: 113 || 182 A $^{\mathrm{v}}$; Schmitz/Mink 1989: 457, 458; BC 3.4: 25, 26 108 D f. $2^{\mathrm{r}}$ (cass. 20 fasc. 76 f. 2); BC 4.3: 154, 156 || 139 K f. $1^{\mathrm{v}}$ (m2, K 9013); Schmitz/Mink 1989: 141; BC 3.3: 44

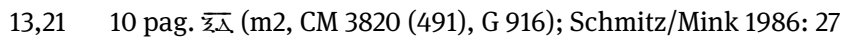
10 pag. $\overline{z 5}$ (m2, CM 3820 (509), G 898) || 108 D f. $3^{\text {r }}$ (cass. 20 fasc. 76 f. 3); BC 4.3: 154, 156 || $124 \mathrm{~K}^{\mathrm{v}}$ (m2, Copte 129,10 f. 161 v); Schmitz/Mink 1986: 
392; BC 3.2: 126, 131 || 139 K f. $2^{\mathrm{r}}$ (m2, K 9014); Schmitz/Mink 1989: 141;

BC 3.3: 44

$\overline{\lambda \Lambda} \quad 14,26 \quad 10$ pag. $\overline{z_{\theta}}$ (m2, CM 3820 (490), G 909); Schmitz/Mink 1986: 27 || 108 D f. $5^{\mathrm{r}}$ (cass. 20 fasc. 76 f. 5); BC 4.3: 154, 156 || 116 Iv ; Schmitz/Mink 1986:

252; BC 3.2: 122 || 124 L f. $1^{\mathrm{r}}$ (Copte 129,10 f. 168); BC 3.2: 126, 131 || $139 \mathrm{~K} \mathrm{f.}$ $4^{\mathrm{v}}$ (m2, K 9016); Schmitz/Mink 1989: 141; BC 3.3: 44 || 286 B (Cod. copt. Bibl. 15 f. 2); Schmitz/Mink 1991: 721; BC 3.4: 35

$15,15 \quad 663^{\mathrm{L}}$ f. $58^{\mathrm{r}}$ (p. <115>; Angabe zum Perikopenincipit)

$\overline{\lambda \varepsilon} \quad 15,26 \quad 10$ pag. $\overline{o r}(\mathrm{~m} 2, \mathrm{CM} 3820$ (516), G 923) || 124 L f. 2v (Copte 129,10 f. 169);

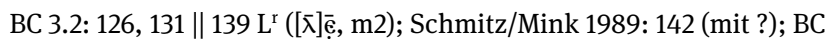
3.3: 44 || $162 \mathrm{D}^{\mathrm{v}}$; BC 3.4: 99 || 286 C f. $6^{\mathrm{v}}$ (Cod. copt. Bibl. 15 f. 8);

Schmitz/Mink 1991: 723; BC 3.4: 35

$\overline{\lambda \Gamma} \quad 17,1 \quad 124 \mathrm{~L} \mathrm{f.} 4^{\mathrm{v}}$ (Copte 129,10 f. 190) BC 3.2: 126, 131

16,30 $115 \mathrm{M}^{\mathrm{v}}$ (m2); Schmitz/Mink 1986: 237; BC 3.2: 105, 112 (irrtümlich $\overline{\lambda \epsilon}$ )

16,33 108 E f. $2^{\mathrm{v}}$ (cass. 20 fasc. 76 f. 8, $\overline{\lambda .}$ ); BC 4.3: 154, 157

18,1 10 pag. $\bar{n}$ (m2, CM 3820 (496), G 915); Schmitz/Mink 1986: 27

$\overline{\lambda z} \quad 18,1 \quad 108$ E f. $4^{r}$ (cass. 20 fasc. 76 f. 10); BC 4.3: 154, 157 || 124 L f. $6^{\mathrm{r}}$ (Copte 129,10 f. 172); BC 3.2: 126, 131 || $292^{\mathrm{L}}$ f. $5^{\mathrm{v}}$, f. $9^{\mathrm{v}}$, f. $13^{\mathrm{r}}$ (an Perikopenanfängen bei 18,1; 18,5 und 18,10, doch mit Bezug auf den gesamten Abschnitt, siehe Anhang 2 - 1.5.); BC 2.2: 61-62, 67

18,15 10 p. $\overline{\mathrm{\Pi B}}$ (m2, CM 3820 (506), G 898); Schmitz/Mink 1986: 27

$\overline{\lambda \mathrm{H}} \quad 18,15 \quad 108 \mathrm{E} \mathrm{f.} 5^{\mathrm{r}}$ (cass. 20 fasc. 76 f. 11); BC 4.3: 154, 157 || $124 \mathrm{~L}$ f. $6^{\mathrm{v}}$ (Copte 129,10 f. 172); BC 3.2: 126, 131|| $260^{\mathrm{r}}$

$\overline{\lambda \Theta} \quad 19,1 \quad * 10$ pag. $\overline{\Pi \Theta}\left(\mathrm{m} 2\right.$, nur Spuren einer Zahl, CM 3820 (?), G 899) \| 108 E f. $7^{\mathrm{r}}$ (cass. 20 fasc. 76 f. 13); BC 4.3: 154, 157 || 116 J'; Schmitz/Mink 1986: 253; BC 3.2: 122 || 124 M f. $1^{\mathrm{v}}$ (m2, Copte 129,10 f. 184); Schmitz/Mink 1986: 394; BC 3.2: 126, 132 || $139 \mathrm{M}^{\mathrm{r}}$ (m2); Schmitz/Mink 1989: 143; BC 3.3: 45 ||

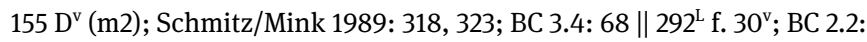
63, 67

19,17 108 E f. $8^{\text {r }}$ (cass. 20 fasc. 76 f. 14); BC 4.3: 154, $157 \| 155$ E f. $1^{\mathrm{r}}(\mathrm{m} 2$, bei aqeı, cass. 24 fasc. 69 f. 3); Schmitz/Mink 1989: 318, 324; BC 3.4: 68

19,16 $116 \mathrm{~J}^{\mathrm{v}}(\mathrm{m} 2$, bei nтоо $\triangle \epsilon, \overline{\mathrm{M}})$; Schmitz/Mink 1986: 254 (irrtümlich für 19,25); BC 3.2: 122 || 124 M f. $1^{\mathrm{v}}$ (m2, vor nTooץ $\Delta \epsilon$, rechts der Kol., Copte 129,10 f. 185); Schmitz/Mink 1986: 394; BC 3.2: 123

$\overline{M \lambda} \quad 19,3810$ pag. $\overline{\Pi \Theta}\left(\mathrm{m} 2\right.$, CM 3820 (507), G 926) \| 155 E f. $2^{\mathrm{r}}$ (m2, cass. 24 fasc. 69 f. 4); Schmitz/Mink 1989: 318, 324; BC 3.4: 68

19,28 108 E f. $9^{\text {r }}$ (cass. 20 fasc. 76 f. 15); BC 4.3: 154, 157

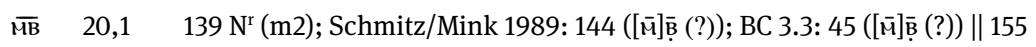
E f. $2^{v}$ (m2, cass. 24 fasc. 69 f. 4); Schmitz/Mink 1989: 318, 324; BC 3.4: 68 || 182 B f. 1'; Schmitz/Mink 1989: 459; BC 3.4: 25, 27 
$\overline{M \Gamma} \quad 20,19 \quad 115 \mathrm{~N}^{\mathrm{r}}$ (rechts der Kol., m2); Schmitz/Mink 1986: 239; BC 3.2: 105, 112 || 116 L f. $1^{v}$ (K 9130, m2); Schmitz/Mink 1986: 256; BC 3.2: 123 (für 20,18) || 182 B f. 2" Schmitz/Mink 1989: 459; BC 3.4: 25, 27 || 369; BC 4.4: 151

पू 20,24 $115 \mathrm{~N}^{\mathrm{r}}(\mathrm{m} 2)$; Schmitz/Mink 1986: 239; BC 3.2: 105, 112 || 116 L f. $1^{\mathrm{v}}$ (K 9130); Schmitz/Mink 1986: 256; BC 3.2: 123 (für 20,23) || 182 B f. $3^{\text {r }}$; Schmitz/Mink 1989: 459; BC 3.4: 25, 27

20,19 663 ${ }^{\mathrm{L}}$ f. $48^{\mathrm{r}}$ (p. <95>; Angabe beim Perikopenincipit)

$\overline{\mathrm{ME}} \quad 21,1 \quad 10$ pag. $\overline{\mathrm{MT}}\left(\mathrm{m} 2, \mathrm{CM} 3820\right.$ (493), G 925); Schmitz/Mink 1986: $27 \| * 115 \mathrm{~N}^{\mathrm{v}}$ [Spuren einer Zahl]; Schmitz/Mink 1986: 239; BC 3.2: 105 (als sichere Lesung angeführt) || 116 L f. $2^{\mathrm{r}}$ (Copte 129,9 f. 48); Schmitz/Mink 1986: 257; BC 3.2: 124 || 182 B f. 3v ; Schmitz/Mink 1989: 459; BC 3.4: 25, 27 || 293 ${ }^{\mathrm{L}} \mathrm{V}^{\mathrm{v}}$ (m2); Schmitz/Mink 1991: 773

21,12 663 f. 48 (p. 96; m2, Angabe beim Perikopenincipit)

In Handschriften überlieferte Titel zu Abschnittsnummern bzw. Kephalaia nach koptischem Modus

\begin{tabular}{|c|c|c|c|}
\hline 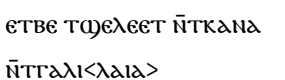 & $\{\bar{\Delta}\}$ & 2,1 & $\begin{array}{l}126 \text { B f. } 1^{v} \text { (Copte 129,9 f. 54); Schmitz/Mink } \\
\text { 1986: 409; BC 3.2: 50-51 }\end{array}$ \\
\hline 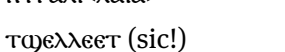 & $\bar{\Lambda}$ & 2,1 & 110 A f. $2^{\mathrm{v}}$ (m2, P. 8775$) ;$ BC 3.2: $86-87$ \\
\hline Етвє пNаY N̄Tачвок & $\{\bar{\varepsilon}\}$ & 2,12 & 126 B f. $2^{\mathrm{r}}$ (Copte 129,9 f. 55); Schmitz/Mink \\
\hline егоүм епрпе · ачтаміє & & & 1986: 409 (zu 2,13); BC 3.2: 50-51 \\
\hline TMacTiz & & & \\
\hline етве NIKO $\triangle$ нмос NTAчеI & $\{\bar{\kappa}\}$ & 3,1 & 126 B f. $2^{v}$ (Copte 129,9 f. 55); Schmitz/Mink \\
\hline ()ג $\overline{\mathrm{IC}} \cdot \overline{\mathrm{NT}} \mathrm{TY}()_{\mathrm{H}}$ & & & 1986: 409; BC 3.2: 50-51 (irrtümlich zu 3,2) \\
\hline$\{\operatorname{EXN} \Theta \lambda H$ & $\overline{1 \theta}$ & $7,37\}$ & $234^{\mathrm{v}}$ [nach BC Titel zu Abschnitts-Nr. $\overline{1}$ ]; \\
\hline & & & Schmitz/Mink 1991: 611 (ohne \\
\hline & & & Interpretation); BC 4.4: 113 \\
\hline 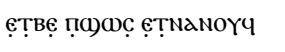 & $\{\overline{\kappa r}\}$ & 10,1 & 126 Eª Schmitz/Mink 1986: 411 (ẹTBẹ, ẹTNa- \\
\hline & & & моҮч); $\mathrm{BC} 3.2:$ 50, 52 (fehlt im $\mathrm{BC}$ ) \\
\hline етве пnаY NTAчNeX & $\{\lambda \bar{\alpha}\}$ & 13,1 & $126 \mathrm{~F}^{\mathrm{r}}$; Schmitz/Mink 1986: 412 (етве, \\
\hline $\operatorname{moọ}[\gamma \in] T \lambda$ eKanн & & & 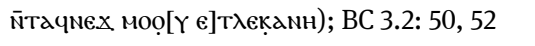 \\
\hline & & & (мооү) \\
\hline ]入̣ Ṇ€ dy . ơ xọ $\bar{\epsilon} \mid[ \pm 2-$ & $\{\overline{\lambda \Gamma}\}$ & 14,1 & $124 \mathrm{~K}^{\mathrm{v}}$ (m2, Copte 129,10 f. $161^{\mathrm{v}}$ ) [Deutung als \\
\hline 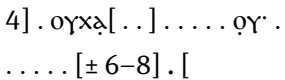 & & & $\begin{array}{l}\text { Titel unsicher]; Schmitz/Mink 1986: } 392 \\
\text { (ohne Lesung) }\end{array}$ \\
\hline$\{€ т в €$ ппарак入нтос & $\overline{\lambda \varepsilon}$ & 15,26 & 124 L f. $2^{v}$ (m2, Copte 129,10 f. 169; Titel des \\
\hline & & & griechischen Kephalaions, zugleich Titel \\
\hline & & & der Abschnitts-Nr.?); BC 3.2: 126, 131$\}$ \\
\hline cion & $\overline{\lambda z}$ & 18,1 & 124 L f. $6^{\mathrm{r}}$ (m2, Copte 129,10 f. 172; zugleich \\
\hline & & & liturgische Rubrik); BC 3.2: 126, 131 \\
\hline
\end{tabular}




\section{A2.5.3. Eusebianische Kanonzahlen}

Nur zwei sahidische Johanneshandschriften sind bisher bekannt, die intermarginal Eusebianische Kanonzahlen aufweisen. Reste von Kanontafeln wurden im Epiphaniuskloster, Theben gefunden. ${ }^{110}$ Die Kodizes 131 und 285 weisen auch griechische Kephalaia und Abschnittsnummern auf. Für 285 ist aufgrund der Manuskriptabmessungen wahrscheinlich, dass es sich um ein für Studienzwecke geschaffenes Exemplar des Johannesevangeliums handeln dürfte, da die Maße für gottesdienstliche Lesung zu klein sind. Die Eusebianische Kanones mögen Vergleichszwecken (zu anderen Sprachtraditionen?) gedient haben. 131 aus dem Weißen Kloster weist ein größeres Format auf, doch scheinen bei dieser Handschrift die unterschiedlichen Methoden der Textstrukturierung durch Nummerierungen Teil der ursprünglichen Anlage der Handschrift gewesen zu sein, was einen Hinweis geben könnte, dass es sich ebenfalls um einen Kodex handelt, der zu Studienzwecken geschaffen wurde.

\begin{tabular}{|c|c|c|}
\hline$\overline{\Pi \mathrm{H}} / \overline{\mathrm{d}}$ & 8,20 & 285 B f. $1^{\text {r }}$ (Hunt. 4, f. 2); BC 3.4: 74, 75 \\
\hline$\overline{\Pi \Theta} / 1$ & 8,21 & 285 Teil B f. $1^{\text {r }}$ (Hunt. 4, f. 2); BC 3.4: 74, 75 \\
\hline$\overline{\mathrm{q}} / \overline{\mathrm{r}}$ & 10,14 & 285 C f. $11^{v}$ (Hunt. 4, f. 14); BC 3.4: 74, 76 \\
\hline$\overline{\varphi र} / \bar{\lambda}$ & 10,15 & 285 C f. $11^{v}$ (Hunt. 4, f. 14); BC 3.4: 74, 76 \\
\hline$\overline{\mathrm{QB}} / \mathrm{i}$ & 10,16 & 285 C f. $11^{v}$ (Hunt. 4, f. 14); BC 3.4: 74, 76 \\
\hline$\overline{\mathrm{q} \Delta} / \mathrm{i}$ & 10,41 & 285 C f. $15^{\mathrm{r}}$ (Hunt. 4, f. 18); BC 3.4: 74, 76 \\
\hline$\overline{\mathrm{\varphi}} / \bar{\lambda}$ & 11,53 & 131 P'; Schmitz/Mink 1989: 21; BC 4.2: 33, 48 \\
\hline$\overline{45} / \bar{\lambda}$ & 11,54 & $131 \mathrm{P}^{\mathrm{r}} ; \mathrm{BC} 4.2: 33,48$ \\
\hline$\overline{\mathrm{qZ}} / \mathrm{i}$ & 11,55 & $131 \mathrm{P}^{\mathrm{r}} ; \mathrm{BC} 4.2: 33,48$ \\
\hline$\overline{\mathrm{qH}} / \bar{\Lambda}$ & 12,2 & $131 \mathrm{P}^{\mathrm{v}} ; \mathrm{BC} 4.2: 33,48$ \\
\hline$\overline{\mathrm{q}} / \mathrm{i}$ & 12,9 & 131 Pv; Schmitz/Mink 1989: 21; BC 4.2: 33, 48 \\
\hline$\overline{\mathrm{P}} / \bar{\alpha}$ & 12,12 & $131 Q^{\text {r; }}$ BC 4.2: 33, 49 || 285 D f. $1^{\text {r }}$ (Hunt. 4, f. 22); BC 3.4: 74, 76 \\
\hline$\{[\overline{\mathrm{pd}} / \overline{\mathrm{z}}]$ & 12,14 & $131 \mathrm{Q}^{\mathrm{r}} ; \mathrm{BC}$ 4.2: 33, 49\} \\
\hline$\overline{\mathrm{PB}} / \mathrm{i}$ & 12,16 & $131 \mathrm{Q}^{\mathrm{r}}$; BC 4.2: 33, 49 || 285 D f. $1^{\mathrm{v}}$ (Hunt. 4, f. 22); BC 3.4: 74, 76 \\
\hline$\overline{\mathrm{P} \Gamma} / \bar{\lambda}$ & 12,23 & $\left\{131 \mathrm{Q}^{\mathrm{v}} ; \mathrm{BC} 4.2: 33,49\right\} \| 285$ D f. $2^{\mathrm{v}}$ (Hunt. 4, f. 23); BC 3.4: 74, 76 \\
\hline$\overline{\mathrm{P} \Lambda} / \mathrm{i}$ & 12,24 & $\left\{131 Q^{\mathrm{v}} ;\right.$ BC 4.2: 33, 49\} || 285 D f. $2^{\mathrm{v}}$ (Hunt. 4, f. 23); BC 3.4: 74, 76 \\
\hline$\overline{\mathrm{PE}} / \overline{\mathrm{r}}$ & 12,25 & $\left\{131 \mathrm{Q}^{\mathrm{v}} ;\right.$ BC $\left.4.2: 33,49\right\} \| 285$ D f. $2^{\mathrm{v}}$ (Hunt. 4, f. 23); BC 3.4: 74, 76 \\
\hline
\end{tabular}

110 New York, Metropolitan Museum of Art, MMA. X.455, siehe Evelyn White/Crum 1926: 122-123 Nr. 584 (Text), 302-305 (Kommentar) mit Tf. 8-9. Die Signaturangabe von Evelyn White und Crum ist heute nicht mehr korrekt. Die zugehörige Evangelienhandschrift, wahrscheinlich ein Tetraevangelium, ist nicht erhalten. Da wohl koptische Nummeralia vorliegen (Verwendung von ч), ist es denkbar, dass es in koptischer Sprache (sahidisch) verfasst war, obgleich der mit den Kanontafeln überlieferte Brief des Eusebius an Carpianus griechisch verfasst ist. 


\begin{tabular}{|c|c|c|}
\hline$\{[\overline{\mathrm{PS}} / \mathrm{i}]$ & 12,26 & $131 Q^{v} ;$ BC 4.2: 33, 49\} \\
\hline$\overline{\mathrm{PZ}} / \bar{\lambda}$ & 12,27 & 131 Qv; Schmitz/Mink 1989: 22; BC 4.2: 33, 49 \\
\hline$\overline{\mathrm{PH}} / \mathrm{i}$ & $12,27 \mathrm{~b}$ & 131 Qv; Schmitz/Mink 1989: 22; BC 4.2: 33, 49 \\
\hline \multirow[t]{2}{*}{$\overline{\mathrm{PN}} / \mathrm{i}$} & 16,33 & 131 R; BC 4.2: 33, 49 („,auf dem abgerissenen Rand“, tatsächlich \\
\hline & vorhanden & \\
\hline \multirow[t]{2}{*}{$\overline{\mathrm{PṆ}} / \overline{\mathrm{T}}^{\text {sic }}$} & 17,1 & $131 \mathrm{R}^{\mathrm{r}}$; Schmitz/Mink 1989: 22 (nennen irrtümlich $\overline{\text { pṆa } / 1) ; ~ B C ~ 4.2: ~}$ \\
\hline & 33, 49 (rek & nstruiert $[\overline{\mathrm{p}} \overline{\mathrm{N}} \Delta / \overline{\mathrm{r}}]$, ,auf dem abgerissenen Rand“) \\
\hline$\overline{\mathrm{CK}} \mathrm{e} / \bar{\Theta}$ & 21,14 & $131 \mathrm{~S}^{\mathrm{r}}$; Schmitz/Mink 1989: 24; BC 4.2: 33, 50 \\
\hline$\overline{\mathrm{CKS}} / \mathrm{i}$ & $21,15 b$ & 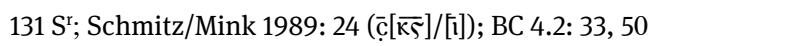 \\
\hline$\overline{\mathrm{CKZ}} / \overline{\mathrm{\theta}}$ & 21,16 & $131 \mathrm{~S}^{\mathrm{r}}$; Schmitz/Mink 1989: 24; BC 4.2: 33, 50 \\
\hline$\overline{\mathrm{CKH}} / \mathrm{i}$ & $21,16 b$ & $131 \mathrm{~S}^{\mathrm{r}}$; Schmitz/Mink 1989: 24; BC 4.2: 33, 50 \\
\hline$\overline{\mathrm{CK} \Theta} / \bar{\Theta}$ & 21,17 & $131 \mathrm{~S}^{\mathrm{r}}$; Schmitz/Mink 1989: 24; BC 4.2: 33, 50 \\
\hline$\overline{\mathrm{c} \lambda} / \mathrm{i}$ & $21,17 \mathrm{~b}$ & 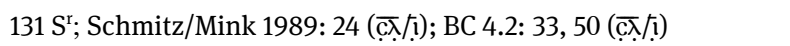 \\
\hline$\overline{c \lambda \lambda} / \bar{\theta}$ & 21,18 & $131 \mathrm{~S}^{\mathrm{r}}$; Schmitz/Mink 1989: $24(\overline{\mathrm{c} \lambda \grave{\alpha}} / \bar{\theta}) ;$ BC 4.2: 33, $50\left(\overline{\mathrm{c} \lambda} \cdot \overline{\mathrm{d}} / \mathrm{e}^{\mathrm{sic}}\right)$ \\
\hline
\end{tabular}

Nota Bene: In BC 3.4: 74, 76 (BC sa 574) wird für 285 bei 8,59 fälschlich $\overline{\mathrm{HB}} / \mathbf{1}$ (sic!) angeführt, was auf einer Fehlinterpreation von $\overline{\mathrm{KB}} / \mathrm{i}$ für Kephalaionzahl nach griechischem Modus und Abschnittsnummer beruht und zu 9,1 gehört. 


\section{A2.5.4. Initialen und textgliedernde Zeichen}

Alle sahidischen Johannes-Kodizes sind auf die eine oder andere Weise durch Initialen oder textgliedernde Zeichen wie Paragraphoi, Diplen, Obeloi oder Koroniden strukturiert; für Proto-Bohairisch und Lykopolitanisch gilt das nur eingeschränkt.

In pbo fehlen Initialen gänzlich, doch Paragraphoi sind zahlreich. Paragraphoi markieren den Ansatz des Schriftspiegels fast aller Seiten, was als Teil des Seitenlayouts bzw. als Markierung für den Kopisten zu werten ist. Einige dieser Paragraphoi fallen dabei mit Textpassagen zusammen, die hervorgehoben werden sollen. An anderen Positionen dienen Paragraphoi der Textgliederung, so bei inhaltlichen Wechseln und bei direkter Rede Jesu sowie Teilen davon. Ungewöhnlich ist die Setzung von Paragraphoi innerhalb des Schriftspiegels (so etwa bei 5,19, 5,24 und 5,25). Sicher sollten sie helfen, Textpassagen schneller zu finden, und die Abschnittsauswahl ist kaum willkürlich. ${ }^{11}$

In den beiden lykopolitanischen Handschriften ist nur an einer Stelle in ly 1 eine Diple klar erkennbar $(6,1)$. Wahrscheinlich sind in ly 1 weitere Stellen gekennzeichnet, doch ist dies weder anhand von Thompsons Edition noch anhand von vorhandenen schwarz-weiß Abbildungen der Handschrift eindeutig zu klären. Für ly 2 sind keinerlei textgliedernde Elemente feststellbar, was nicht unbedingt verwundert, da es sich um einen literarischen Text in einem dokumentarischen Manuskript handelt.

In sahidischen Manuskripten werden koronis-, paragraphos-, obelos- und diplenartige Zeichen zur Textgliederung (nebeneinander) verwendet. Meist begleiten sie Initialen als Zierelemente, seltener treten sie alleine auf oder weisen innerhalb einer Handschrift besondere Formen zueinander auf. ${ }^{112}$ Am häufigsten dienen Koronis und Diple als Paragraphenzeichen, wobei eine Abgrenzung teils dadurch erschwert wird, dass der Ansatz von Koroniden häufig diplenförmig gestaltet ist. ${ }^{113}$

Die Größe von Initialen variiert stark. In einigen Manuskripten ist der Unterschied zu normal großen Buchstaben so fließend, dass eine Systematisierung

111 Fallen Vers- und Seitenbeginn zusammen, sind sie in der Übersichtstabelle in geschweifte Klammern gesetzt.

112 In 106 sind Paragraphoi bei 3,3; 8,46 und 9,27, in 108 bei 17,9 besonders hervorgehoben. Der Grund ist unbekannt.

113 Die maßgebliche Arbeit zu Form und Entwicklung von Paragraphenzeichen koptischer Handschriften ist Petersen 1954. Petersen nennt Diple und Koronis als Paragraphenzeichen, Petersen 1954: 295-330, doch sind auch Paragraphos (horizontale Linie mit hakenförmigem Ansatz) und Obelos (horizontale Linie begleitet von Punkten ober- und unterhalb) gut belegt. 
kaum möglich ist, besonders bei kalligraphisch einfach ausgeführten Manuskripten wie 108, 115 oder 123. Gerade 115 ist bei Aussetzung und Größe von Initialen so nuanciert, dass es oft unmöglich ist zu sagen, was zufällig und was beabsichtigt und daher möglicherweise für die Interpretation von Bedeutung ist.

In einigen Kodizes ist eine sehr feine Gliederung des Inhalts durch Initialen und textgliedernde Zeichen feststellbar. Zwei Beispiele mögen das illustrieren:

In 109 strukturieren punktierte Obeloi im Verbund mit Initialen den Text nach Sprechern und Handelnden und gliedern längere Monologe nach dem Sinn. Die Setzung der Obeloi lässt sich kaum anders erklären, als dass die Handschrift für liturgischen Vortrag eingerichtet wurde, sodass ein nach Personen und Sinneinheiten gegliederter Vortrag möglich war. Nicht für alle mehr oder minder erhaltenen Verse ist eine Aufteilung vorhanden oder noch ersichtlich, da einige Verse unbezeichnet sind und Teile der Ränder mit Verlust der Zeilenanfänge ausgebrochen sind:

\begin{tabular}{|c|c|c|}
\hline & & Sprechende/Handelnde/Sinnabschnitte \\
\hline & {$[1,1-8,56$} & Lacuna] \\
\hline$\div$ & 8,57 & Juden \\
\hline$\div$ & 8,58 & Jesus \\
\hline & {$[8,59$} & $\div$ vielleicht in Lacuna] \\
\hline Ini. & $9,1-2$ & Bericht und Jünger \\
\hline & $9,2-5$ & unbezeichnet \\
\hline$\div$ & 9,6 & Heilung eines Blinden \\
\hline & {$[9,7-14$} & $\div$ vielleicht in Lacuna] \\
\hline$\div$ & 9,15 & Pharisäer \\
\hline$\div$ & 9,16 & einige Pharisäer \\
\hline$\div$ & 9,17 & Pharisäer \\
\hline$\div$ & 9,17 & Blindgeborener \\
\hline & {$[9,18-15,16$} & Lacuna] \\
\hline$\div$ & 15,17 & Jesus (Bildrede vom Fruchtbringen) \\
\hline$\div$ & 16,4 & Jesus (Rede vom Paraklet) \\
\hline & {$[16,5-21,25$} & Lacuna] \\
\hline
\end{tabular}

Zwei längere, durch Initialen und Obeloi gegliederte Abschnitte überliefert 112. Obeloi und alle Interpunktionszeichen (meist Punkte, seltener Spiegelstriche) sind in einer vom Text abweichenden silbrig-grauen Tinte geschrieben, so auch Zierelemente der Kopfzeilen und Auszierungen bei Initialen. Wahrscheinlich stammen sie von einer zweiten Hand. Interessanterweise fehlt bei $112 \mathrm{~S}$ f. $1^{\mathrm{r}}$ (Wien, ÖNB, K 9083') silbrig-graue Tinte und somit fehlen auch Obeloi, Interpunktion oder Zierelemente. Es lässt sich mutmaßen, dass der Schreiber, der die Handschrift mit Verzierungen versah, die Seite überblättert hat. 
I.

\begin{tabular}{|c|c|c|}
\hline & & Sprechende/Handelnde/Sinnabschnitte \\
\hline & {$[1,1-3,24$} & Lacuna] \\
\hline$\div$ & 3,25 & Bericht \\
\hline$\div$ & 3,26 & Bericht, Jünger Johannes des Täufers und seine Antwort \\
\hline$\div$ & 3,29-30 & am Versende vor єтє паı пє, Bericht \\
\hline$\div$ & $\begin{array}{l}3,31 \\
{[3,32-4,6}\end{array}$ & $\begin{array}{l}\text { Johannes der Täufer } \\
\div \text { vielleicht in Lacuna] }\end{array}$ \\
\hline Ini. & $4,7-8$ & bei пexe $\overline{\mathrm{cc}} \mathrm{nac}$, Jesus \\
\hline$\div$ & $\begin{array}{l}4,9 \\
{[4,10-12}\end{array}$ & $\begin{array}{l}\text { Samaritanerin } \\
\div \text { vielleicht in Lacuna] }\end{array}$ \\
\hline$\div$ & $4,13-14$ & Jesus \\
\hline$\div$ & 4,15-16 & Samaritanerin, dann Jesus \\
\hline$\div$ & $\begin{array}{l}4,16 \\
{[4,17-18}\end{array}$ & $\begin{array}{l}\text { Versende, Samaritanerin } \\
\div \text { vielleicht in Lacuna] }\end{array}$ \\
\hline$\div$ & $4,19-20$ & Samaritanerin \\
\hline$\div$ & 4,21-22 & Jesus \\
\hline$\div$ & $4,22-23$ & bei anọn $[\Delta \epsilon \in$ eno $] \gamma \omega \alpha$, Jesus \\
\hline$\div$ & $\begin{array}{l}4,23 \\
{[4,24-28}\end{array}$ & $\begin{array}{l}\text { bei epe п! }[\text { [т гар ()ınє], Jesus } \\
\div \text { vielleicht in Lacuna] }\end{array}$ \\
\hline Ini. & $\begin{array}{l}4,28 \\
{[4,29-30}\end{array}$ & $\begin{array}{l}\text { bei пехас мN̄мрсме, Samaritanerin } \\
\div \text { vielleicht in Lacuna] }\end{array}$ \\
\hline Ini. & $4,31-33$ & Bericht, Jünger, nur kurz Jesus \\
\hline Ini. & $4,34-35$ & Jesus \\
\hline Ini. & $4,35-36$ & bei єІс әннте, Jesus \\
\hline$\div$ & $\begin{array}{l}4,36 \\
{[4,37-6,25}\end{array}$ & 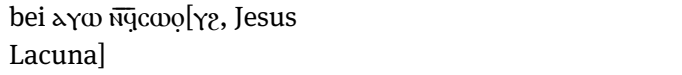 \\
\hline
\end{tabular}

II.

\begin{tabular}{|c|c|c|}
\hline & & Sprechende/Handelnde/Sinnabschnitte \\
\hline$\div$ & 6,27 & bei $2 \lambda \lambda d$ тє२рє ஸ்точ, Jesus \\
\hline$\div$ & $6,28-29$ & eine Menge in Kapharnaum, Jesus \\
\hline$\div$ & $6,30-31$ & eine Menge in Kapharnaum \\
\hline$\div$ & 6,32 & Jesus \\
\hline$\div$ & 6,33 & Jesus \\
\hline$\div$ & 6,34 & eine Menge in Kapharnaum \\
\hline$\div$ & 6,35 & Jesus \\
\hline$\div$ & $6,36-37$ & Jesus \\
\hline$\div$ & $6,37-39$ & bei аүс пєтннү ()аpol, Jesus \\
\hline$\div$ & 6,40 & Jesus \\
\hline Ini. & 6,41 & indirekte Rede \\
\hline$\div$ & 6,42 & Juden \\
\hline$\div$ & $6,43-44$ & Jesus \\
\hline$\div$ & 6,45 & Jesus/AT-Zitat \\
\hline \multirow[t]{2}{*}{$\div$} & 6,45 & bei оүом мім мंтачсळтм', Jesus \\
\hline & {$[7,23-29$} & Seite vom Setzer der Auszierungen übersprungen] \\
\hline
\end{tabular}




\begin{tabular}{lll}
\hline & \multicolumn{3}{c}{ Sprechende/Handelnde/Sinnabschnitte } \\
\hline Ini. & 7,30 & Bericht \\
& {$[7,31$} & Zeilenanfänge in Lacuna] \\
Ini. & 7,32 & Bericht über Pharisäer und Hohepriester \\
Ini. & $7,33-34$ & Jesus \\
$\div$ & $7,35-36$ & Juden \\
Ini. & $7,37-39$ & Jesus \\
$\div$ & 7,40 & einige \\
$\div$ & 7,41 & andere \\
$\div$ & 7,42 & AT-Zitat \\
$\div$ & 7,43 & Bericht \\
Ini. & 7,44 & einige, $\div$ begonnen, doch unvollendet \\
$\div$ & 7,45 & Knechte \\
$\div$ & 7,45 & bei nexє NH NaY xe, Knechte \\
$\div$ & $7,46-47$ & Knechte und Pharisäer, vielleicht bis 7,49 \\
& {$[7,48-49$} & $\div$ vielleicht in Lacuna] \\
$\div$ & $7,50-51$ & Nikodemus \\
$\div$ & 7,52 & einige aus dem Volk \\
& $(7,53-8,11$ & nicht in sahidischen Handschriften) \\
$\div$ & 8,12 & Jesus \\
$\div$ & 8,13 & Pharisäer \\
& {$[8,14-21,25$} & Lacuna]
\end{tabular}

Die Textgliederung orientiert sich offensichtlich an Akteuren und erklärenden Passagen. Da eine solche Feingliederung nicht für stilles Lesen oder den gottesdienstlichen Vortrag als Perikope durch einen einzelnen Lektor benötigt wird, ist an eine Verteilung auf mehrere Personen zu denken. Es muss offenbleiben, wie genau sich der Vortrag gestaltete. Denkbar ist sowohl eine einfache Verteilung auf mehrere Diakone oder Lektoren als auch ein (szenischer) Vortrag mit Rollenverteilung. Vergleichbar gegliedert sind 106, 115, 124, 125 134, 139, 174 und CGC 8086. In 103, 108, 123, 126, 127, 131, 132, 136, 140, 141, 154, 167, 173 und 199 treten Initialen und textgliedernde Zeichen unregelmäßiger auf, doch eine ähnliche Funktion erscheint möglich. Für 142, 182, 197, 234, 235, 238, 250, 260 und 369 ist der Befund aufgrund der Erhaltung unsicher. Für 285 ist die Einteilung neben enthaltenen Nummerierungen von besonderem Interesse, da die Handschrift zum liturgischen Vortrag aufgrund ihrer Größe eher ungeeignet ist. Es mag sein, dass gerade 285 auch als Studienhandschrift für die Ausbildung zum liturgischen Vortrag konzipiert wurde.

Als generelle Tendenz ist eine Zunahme an Initialen und Ornamenten erkennbar, je jünger ein Manuskript datiert wird (teils durch Kolophone belegbar). Die laut Literatur ältesten Manuskripte weisen nur wenige markierte Text- 
abschnitte auf, die meist mit Perikopenanfängen liturgischer Handschriften zusammenfallen. ${ }^{114}$

Trotz Clusterungen im handschriftlichen Befund muss der fragmentarische Zustand des Materials bedacht werden. So sind 2,10 von 23 oder 16,4 von 24 sahidischen Handschriften bezeugt, doch 5,12 nur von 9 und 14,3 von 10. Insgesamt erscheint es sinnvoll, neben speziellen Untersuchungen, die Gruppierungen von Manuskripten aufgrund ähnlicher Einteilungen versuchen und darauf aufbauend Ableitungen vornehmen, gerade diejenigen Verse in den Blick zu nehmen, die keinerlei besondere Hervorhebung aufweisen. Sie erlauben Aussagen, welche Verse in moderer Einteilung nach sahidisch-koptischer Tradition mit höchster Wahrscheinlichkeit nicht als eigenständig, sondern als zu Texteinheiten ihres Umfeldes gehörig angesehen wurden.

114 Eine Aufschlüsselung bietet Schulz 2021. 
A2.5.5. Tabellarische Übersicht zu Paratexten

\begin{tabular}{|c|c|c|c|}
\hline & Paragraphenzeichen & Initialen & Nummerierungen / Varia \\
\hline 1,1 & $\begin{array}{l}\text { pbo, 4, 5, } 10(\stackrel{*}{*}), 40(\dot{*}), \\
106(\stackrel{*}{*}), 134(\dot{*})\end{array}$ & $\begin{array}{l}\text { 4, 5, } 9 \text { (vgr.), } 10 \text { (vgr.), } 19 \\
\text { (vgr.), 40, 106, } 134 \text { (vgr.) }\end{array}$ & \\
\hline \multicolumn{4}{|c|}{ 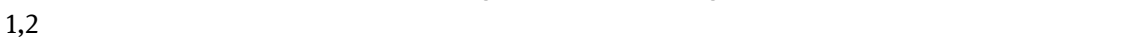 } \\
\hline \multicolumn{4}{|l|}{1,3} \\
\hline 1,4 & & 19 & \\
\hline \multicolumn{4}{|l|}{1,5} \\
\hline 1,6 & 1, $10(\dot{*}), 106(\dot{*}), 134(\dot{*})$ & $\begin{array}{l}\text { 4, } 9 \text { (vgr.), } 10 \text { (vgr.), 19, } \\
\text { 106, } 134 \text { (vgr.), *139 }\end{array}$ & Abschnitt 1: 134 \\
\hline \multicolumn{4}{|c|}{ 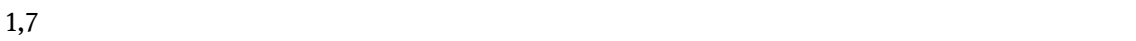 } \\
\hline \multicolumn{4}{|l|}{1,8} \\
\hline \multicolumn{4}{|l|}{1,9} \\
\hline \multicolumn{4}{|l|}{1,10} \\
\hline 1,11 & & 139 & \\
\hline \multicolumn{4}{|l|}{1,12} \\
\hline \multicolumn{4}{|l|}{1,13} \\
\hline 1,14 & $181(>)$ & 19 (vgr.), 103, 134 & \\
\hline 1,15 & $4($ (২), $10(\dot{*}), 134(\dot{*}), 181(>)$ & $\begin{array}{l}4 \text { (vgr.), } 9 \text { (vgr.), } 10 \text { (vgr.), } \\
\text { 19, } 134 \text { (vgr.), } 181\end{array}$ & \\
\hline \multicolumn{4}{|c|}{ - } \\
\hline \multicolumn{4}{|l|}{1,17} \\
\hline 1,18 & $134(\dot{*})$ & 9 (vgr.), 19, 134 (vgr.) & Abschnitt 2: 134 \\
\hline 1,19 & $10(\dot{x})$ & $\begin{array}{l}10 \text { (vgr.), 19, } 40 \text { (vgr.), 134, } \\
139\end{array}$ & \\
\hline \multicolumn{4}{|c|}{-6} \\
\hline 1,21 & & 19,139 & \\
\hline 1,22 & & 40 (vgr.), 134 & \\
\hline 1,23 & & $19,40, * 47(?), 134$ & $\begin{array}{l}\text { Zitat } \rightarrow »: 134 \text {; Zitat } \rightarrow \\
\text { Punkte: } 181\end{array}$ \\
\hline 1,24 & $3(>), 116(\div)$ & 116 (vgr.), 134, 139 & \\
\hline \multicolumn{4}{|l|}{1,25} \\
\hline 1,26 & $110(\div)$ & $110,115,126$ & \\
\hline 1,27 & 12 & 12 & \\
\hline 1,28 & 117 (gr) & $9,10,115,117$ (gr) & \\
\hline 1,29 & $4(>), 5(>/ \odot), 10(\dot{*}), 116(\dot{*})$ & $\begin{array}{l}\text { 4, 5, 9, } 10 \text { (vgr.), 19, } 110 \\
\text { (vgr.), } 115 \text { ( } 2 \times \text {, Beginn und } \\
\text { аүळ пехач), 116, } 139\end{array}$ & $\begin{array}{l}\text { Glosse: } 110 \text { (товє <є>твє п- } \\
\text { вдптісмд, m2, † für den } 11 . \\
\text { Tobe) }\end{array}$ \\
\hline 1,30 & $115,181(\dot{*})$ & 115 & \\
\hline 1,31 & & 110,115 & \\
\hline 1,32 & & 115 & \\
\hline 1,33 & & 115 (bеi паı пєтнаваптіzе) & \\
\hline 1,34 & $116(\div)$ & 115,116 & \\
\hline
\end{tabular}




\begin{tabular}{|c|c|c|c|}
\hline & Paragraphenzeichen & Initialen & Nummerierungen / Varia \\
\hline 1,35 & $\begin{array}{l}3(>), 4(>), 5(>/ \odot), 10(\cdot \dot{*}), \\
* 115,116(\cdot \dot{*})\end{array}$ & $\begin{array}{l}\text { 4, 5, 9, } 10 \text { (vgr.), 19, 40, } 110 \\
\text { (vgr.), 115, } 116 \text { (vgr.), } 126 \\
\text { (vgr.) }\end{array}$ & $\begin{array}{l}\text { Abschnitt 3: 110, }\{115(\mathrm{~m} 2)\} \text {, } \\
116\end{array}$ \\
\hline 1,36 & & 115 & \\
\hline 1,37 & $110(\div)$ & 110,115 & \\
\hline 1,38 & $126(\div$ bei пехаY Nay $)$ & $\begin{array}{l}\text { 10, } 19 \text { (bei пєхау Nay), } 115 \\
(2 \times, \text { Beginn und пєхаY } \\
\text { Nay), } 126 \text { (bei пєхаҮ Nay) }\end{array}$ & \\
\hline 1,39 & $126(\div)$ & 115,126 & \\
\hline 1,40 & $110(\div), 126(\div)$ & 110 (vgr.), 115, 126 & $\begin{array}{l}\text { Glosse: } 110 \text { (тавє, m2, } \neq \text { für } \\
\text { 12. Tobe) }\end{array}$ \\
\hline \multicolumn{4}{|l|}{1,41} \\
\hline 1,42 & $110($ bei $\overline{\mathrm{lc}} \Delta \mathrm{\epsilon})$ & $\begin{array}{l}19 \text { (bei } \overline{\mathrm{ic}} \Delta \epsilon), 110 \text { (bei } \overline{\mathrm{ic}} \\
\Delta \epsilon), 115 \text { (bei } \overline{\mathrm{ic}} \Delta \epsilon)\end{array}$ & \\
\hline 1,43 & 4, $110(\div), 116(\div), 215(\div)$ & $\begin{array}{l}\text { 4, 9, } 10 \text { (vgr.), 19, } 110 \text { (vgr.), } \\
\text { 115, } 116 \text { (vgr.), } 126 \text { (vgr.), } \\
215 \text { (vgr.) }\end{array}$ & 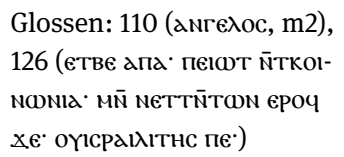 \\
\hline 1,44 & & 110,115 & \\
\hline 1,45 & $126(\div)$ & 115 & \\
\hline 1,46 & $\begin{array}{l}126(2 \times, \div \div, \text { Beginn und } п є- \\
x \in \text { філиппос) }\end{array}$ & 19, 115 & \\
\hline 1,47 & $126(\div)$ & $115,126, * 136$ & \\
\hline 1,48 & 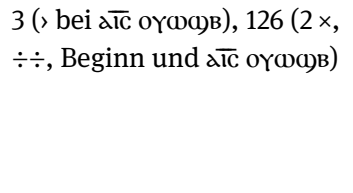 & 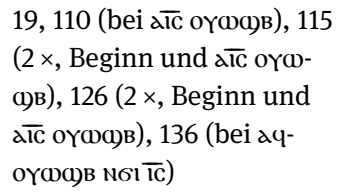 & \\
\hline 1,49 & $126(\div)$ & $115,126,167$ & \\
\hline 1,50 & $3(>), 126(\div), 136(\cdot \dot{*})$ & $19,115,126,167$ & \\
\hline 1,51 & & $115,126,167$ & „Amen-Wort“ 1: 4 \\
\hline 2,1 & $\begin{array}{l}1,4(>), 10(\cdot \dot{*}), 47(>), 115 \\
(\div), 116(\cdot \dot{*}), 126(\cdot \dot{*}), 136 \\
(\cdot \dot{*}), 167(\cdot \dot{*}), 181(\odot / \cdot \dot{*})\end{array}$ & $\begin{array}{l}\text { 4, } 9 \text { (vgr.), } 10 \text { (vgr.), } 19 \\
\text { (vgr.), } 40 \text { (vgr.), 47, } 110 \\
\text { (vgr.), 115, } 116 \text { (vgr.), 126, } \\
\text { 167, } 181 \text { (vgr.) }\end{array}$ & 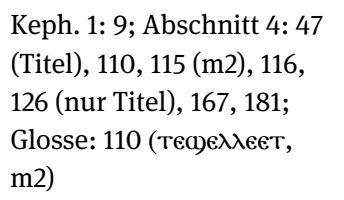 \\
\hline 2,2 & & 115 & \\
\hline 2,3 & & 115 & \\
\hline 2,4 & $126(\div)$ & 115 & \\
\hline 2,5 & $126(\div)$ & 115,126 & \\
\hline 2,6 & $116(\div), 126(\div)$ & $115,116,126$ & \\
\hline 2,7 & $115(\cdot \dot{*}), 116,126(\div)$ & $19,115,116,126$ & \\
\hline 2,8 & & $\begin{array}{l}115(2 \times \text {, Beginn und NToor } \\
\Delta \epsilon)\end{array}$ & \\
\hline
\end{tabular}




\begin{tabular}{|c|c|c|c|}
\hline & Paragraphenzeichen & Initialen & Nummerierungen / Varia \\
\hline 2,9 & 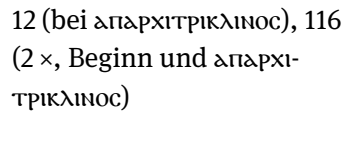 & 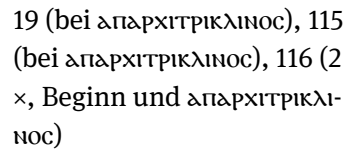 & \\
\hline 2,10 & & 115 (bei мток $\triangle €$ ак२арє२) & \\
\hline 2,11 & $3(>)$ & 115,136 & \\
\hline 2,12 & $\begin{array}{l}\text { 1, } 3(>), 4(>), 5,10(\dot{*}), 116 \\
(\cdot \dot{*}), 126(\div), 167(\dot{*}), 181(>)\end{array}$ & $\begin{array}{l}\text { 4, } 9 \text { (vgr.), } 10 \text { (vgr.), } 19 \\
\text { (vgr.), } 110 \text { (vgr.), 115, } 116 \\
\text { (vgr.), } 126 \text { (vgr.), } 136 \text { (vgr.), } \\
\text { 167, } 181 \text { (vgr.) }\end{array}$ & $\begin{array}{l}\text { Keph. 2: 9, }\{10 \text { (rezent) }\}, 47 \\
\text { (Titel); Abschnitt 5: 110, } 115 \\
\text { (m2), 116, } 126 \text { (nur Titel), } \\
\text { 167, } 181\end{array}$ \\
\hline 2,13 & 5, CGC $8086(\dot{*})$ & $\begin{array}{l}\text { 115, 136, *167, 174, CGC } \\
8086 \text { (vgr.) }\end{array}$ & \\
\hline \multicolumn{4}{|l|}{2,14} \\
\hline \multicolumn{4}{|l|}{2,15} \\
\hline 2,16 & & 19,115 & \\
\hline 2,17 & & 115 & Zitat $\rightarrow »: 1,4,10$ \\
\hline 2,18 & $3(>), 116,126(\div)$ & $\begin{array}{l}\text { 19, 115, 116, 126, 136, 167, } \\
\text { 174, CGC } 8086 \text { (vgr.) }\end{array}$ & \\
\hline 2,19 & $\begin{array}{l}106(\dot{*}), 116(\dot{*}), 126(\div), \\
167(\dot{*})\end{array}$ & $\begin{array}{l}106 \text { (vgr.), 115, } 116 \text { (vgr.), } \\
126,136,167\end{array}$ & \\
\hline 2,20 & $126(\div)$ & 115,126 & \\
\hline 2,21 & & 115, CGC 8086 & \\
\hline 2,22 & 106 (bеi аүпістеүє, $\stackrel{*}{*}$ ) & 115, CGC 8086 & \\
\hline 2,23 & $10(\dot{*}), 12,126(\div)$ & $\begin{array}{l}9 \text { (vgr.), } 10 \text { (vgr.), } 19 \text { (vgr.), } \\
\text { 115, 116, } 126 \text { (vgr.), 136, 167, } \\
174\end{array}$ & \\
\hline 2,24 & & 115 & \\
\hline \multicolumn{4}{|l|}{2,25} \\
\hline 3,1 & 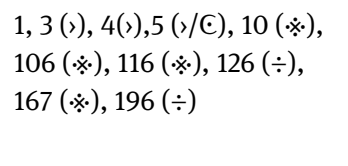 & $\begin{array}{l}\text { 4, 5, } 9 \text { (vgr.), } 10 \text { (vgr.), 19, } \\
88 \text { (vgr.), 106, } 115,116 \\
\text { (vgr.), 126, } 136 \text { (vgr.), 167, } \\
174 \text { (vgr.), } 196\end{array}$ & $\begin{array}{l}\text { Keph. 3: 9, }\{10 \text { (rezent)\}; } \\
\text { Abschnitt 6: *10 ( }) \text { ) } 115 \\
(\mathrm{~m} 2), 116,126 \text { (nur Titel), } \\
167\end{array}$ \\
\hline \multicolumn{4}{|c|}{ 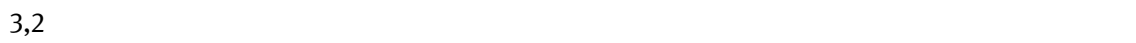 } \\
\hline 3,3 & $106(\bigodot), 126$ & $\begin{array}{l}19,106,115,126,136,167, \\
174\end{array}$ & „Amen-Wort“ 2: 4 \\
\hline 3,4 & $106(\dot{*}), 116$ & $106,115,116,136,174$ & \\
\hline 3,5 & $106(\div), 116(\div$ und $C)$ & $\begin{array}{l}19 \text { (vgr.), 106, 115, 116, 136, } \\
167,174\end{array}$ & „Amen-Wort“3: 4 \\
\hline 3,6 & & 115 & \\
\hline 3,7 & 116 & 115,116 & \\
\hline 3,8 & 12 & $\begin{array}{l}115(2 \times, \text { Beginn und } 2 \lambda \lambda d \\
\text { мгсооүN) }\end{array}$ & \\
\hline 3,9 & $10(\dot{\times}), 116$ & $\begin{array}{l}10 \text { (vgr.), } 19 \text { (vgr.), 115, 116, } \\
136,167\end{array}$ & \\
\hline 3,10 & $116(\div$ und $C)$ & $115,116,136$ & \\
\hline
\end{tabular}




\begin{tabular}{|c|c|c|c|}
\hline & Paragraphenzeichen & Initialen & Nummerierungen / Varia \\
\hline 3,11 & $3(>), 4(>), 106(\cdot \dot{*}), 136(\cdot \dot{*})$ & $\begin{array}{l}\text { 4, 106, } 115 \text { (vgr.), } 136 \text { (vgr.), } \\
167\end{array}$ & „Amen-Wort“ 4: 4 \\
\hline 3,12 & 116 & 115,136 & $\begin{array}{l}\text { ł: } 116(\triangle, \text { Kreuz und Punk- } \\
\text { te, } \mathrm{m} 2)\end{array}$ \\
\hline 3,13 & $\begin{array}{l}136(\cdot \dot{x} \text { beim letzten Wort } \\
\text { von } 3,12 \text {, wohl für } 3,13)\end{array}$ & & \\
\hline $\begin{array}{l}3,14 \\
3,15\end{array}$ & $116(\div$ und $\odot), 181(>)$ & $115,116,167$ & \\
\hline 3,16 & $\begin{array}{l}10(\cdot \dot{x}), 106,116(\cdot \dot{x}), 136 \\
(\cdot \dot{*}), 167(\cdot \dot{*})\end{array}$ & $\begin{array}{l}9 \text { (vgr.), } 10 \text { (vgr.), 19, 106, } \\
116 \text { (vgr.), 136, } 137 \text { (vgr.), } \\
167\end{array}$ & \\
\hline 3,17 & & 115, 136 (vgr.), 167 & \\
\hline 3,18 & & 19 & \\
\hline 3,19 & 116 & $\begin{array}{l}19 \text { (vgr.), } 105 \text { (vgr.), 115, } \\
116,167\end{array}$ & \\
\hline 3,20 & 141 (Strich) & $\begin{array}{l}115(2 \times \text {, Beginn und a yod } \\
\text { meqє1), } 136 \text { (vgr.), } 141\end{array}$ & \\
\hline 3,21 & & & \\
\hline 3,22 & $\begin{array}{l}1(>), 3(>), 4(>), 5(>/ \bigodot), * 10 \\
\text { (Reste von } \dot{*} \cdot \text {, hierher?), } 116 \\
(\cdot \dot{*}), 181(\cdot \dot{*})\end{array}$ & $\begin{array}{l}\text { 4, 5, 9, *10 (Reste von } * \dot{*}, \\
\text { hierher?), 19, 115, } 116 \text { (vgr.), } \\
136 \text { (vgr.), 167, } 181 \text { (vgr.) }\end{array}$ & $\begin{array}{l}\text { Abschnitt 7: } 115 \text { (m2), } 116 \\
(\mathrm{~m} 2), 167,181\end{array}$ \\
\hline $\begin{array}{l}3,23 \\
3,24\end{array}$ & 141 (Strich) & 115,141 & \\
\hline 3,25 & $\begin{array}{l}3(>), 112\left(\text { (ा } \text { und }^{\circ \circ}\right), 115(\cdot \dot{*}) \text {, } \\
141(>)\end{array}$ & $\begin{array}{l}9 \text { (vgr.), 112, } 115 \text { (vgr.), } 141 \\
\text { (vgr.) }\end{array}$ & \\
\hline 3,26 & & $\begin{array}{l}\text { 112, } 115 \text { (bei єıс 2ннте), } 149 \\
\text { (bei єıс гннтє), } 167\end{array}$ & \\
\hline 3,27 & $12(\dot{x}), 116(\dot{*})$ & $12,19,115,116$ & \\
\hline 3,28 & & 115,136 & \\
\hline 3,29 & $\begin{array}{l}5(>/ \mathcal{C}), 112\left(\boldsymbol{\Phi} \text { und }^{\circ \circ}\right), 141 \\
(>)\end{array}$ & $\begin{array}{l}\text { 5, } 112 \text { ( } 2 \text { ×, Beginn und па- } \\
\text { pда)є 6є), } 115 \text { (bei парас) } \\
\text { бє), } 136 \text { (bei єтє паl пє, } \\
\text { wohl für парас)є бє), } 141 \\
\text { (vgr.) }\end{array}$ & \\
\hline 3,30 & $116,141(>)$ & 19,116 & \\
\hline 3,31 & $\begin{array}{l}110(\div), 112\left(\mathbb{\Phi} \text { und }^{\circ \circ}\right), 141 \\
\text { (Strich) }\end{array}$ & $\begin{array}{l}19 \text { (bei пємтдчє } \Delta є є \text { єво } \lambda \text { 2N } \\
\text { тпє, vielleicht zu 3,32?), } \\
110,112,115\end{array}$ & \\
\hline 3,32 & $141(>)$ & & \\
\hline 3,33 & $141(>)$ & 115 & \\
\hline 3,34 & $141(>)$ & $\begin{array}{l}115(2 \times \text {, Beginn und Nєрєп- } \\
\text { NoҮт }), 167\end{array}$ & \\
\hline 3,35 & $\begin{array}{l}136(\cdot \dot{x} \cdot \text { beim letzten Wort } \\
\text { von } 3,34 \text {, wohl } z u 3,35)\end{array}$ & 115 & \\
\hline 3,36 & $\begin{array}{l}116(\div \text { bеi пєтмчпістєүє), } \\
141\end{array}$ & 115, 116 (bei пєтмчпитеүє) & \\
\hline
\end{tabular}




\begin{tabular}{|c|c|c|c|}
\hline & Paragraphenzeichen & Initialen & Nummerierungen / Varia \\
\hline 4,1 & 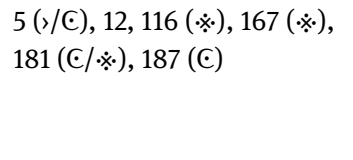 & $\begin{array}{l}\text { 5, } 9 \text { (vgr.), } 10 \text { (vgr.), 19, } 110 \\
\text { (vgr.), 115, } 116 \text { (vgr.), } 136 \\
\text { (vgr.), } 139 \text { (vgr.), } 141 \text { (vgr.), } \\
\text { 167, } 181 \text { (vgr.) }\end{array}$ & $\begin{array}{l}\text { Keph. 4: 9; Abschnitt 8: } \\
\text { 110, } 115 \text { (m2, rechts der } \\
\text { Kol.), 116, } 139(\mathrm{~m} 2), 167 \text {, } \\
\text { 181; Abschnitt 9: } 12\end{array}$ \\
\hline 4,2 & & 115 & \\
\hline \multicolumn{4}{|l|}{4,3} \\
\hline \multicolumn{4}{|l|}{4,4} \\
\hline 4,5 & $10(\dot{*}), 116(\dot{*}), 139(\dot{*})$ & $\begin{array}{l}9 \text { (vgr.), } 10 \text { (vgr.), } 19 \text { (vgr.), } \\
\text { 115, } 116 \text { (vgr.), } 136 \text { (vgr.), } \\
\text { 139, } 141 \text { (vgr.), } 167\end{array}$ & \\
\hline 4,6 & $3(>$ bei $\overline{\imath c} 6 \boldsymbol{c})$ & $115($ bei $\overline{\imath c} \sigma \epsilon)$ & \\
\hline 4,7 & & $\begin{array}{l}112(2 \times \text {, Beginn und пєхє } \overline{\mathrm{lc}} \\
\text { Nac), 115, 136, } 142 \text { (bei пєхє } \\
\overline{\mathrm{IC}} \text { Nac) }\end{array}$ & \\
\hline \multicolumn{4}{|l|}{4,8} \\
\hline 4,9 & $\begin{array}{l}112(\div), 116(\div \text { und } \odot), * 139 \\
\text { (Spuren von } \dot{*} \text { ) }\end{array}$ & $\begin{array}{l}\text { 19, 112, 115, 116, } 142 \text { (bei } \\
\text { мас) мє коүєа)) }\end{array}$ & \\
\hline 4,10 & $116(\div$ und $\odot)$ & $\begin{array}{l}\text { 19, 115, 116, 124, } 142(2 \times \\
\text { Beginn, vgr., und } x \in \text { aүeic) }\end{array}$ & \\
\hline 4,11 & $116(\div$ und $\odot)$ & $19,115,116,124,142$ & \\
\hline 4,12 & & & \\
\hline 4,13 & $112\left(\right.$ (ा und $\left.^{\circ \circ}\right), 116,167(\stackrel{*}{*})$ & $19,112,116,124,167$ & \\
\hline 4,14 & & 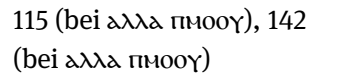 & \\
\hline 4,15 & 112 (II und $\left.{ }^{\circ \circ}\right), 116$ & $12,19,112,116,124,167$ & \\
\hline 4,16 & 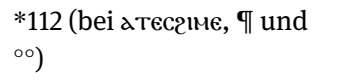 & 112 (bei атесгіме), 115, 124 & \\
\hline 4,17 & & 115 & \\
\hline 4,18 & & & \\
\hline 4,19 & $112\left(\boldsymbol{\Phi}\right.$ und $\left.^{\circ \circ}\right), 116$ & $19,112,115,116,190$ & \\
\hline 4,20 & & $\begin{array}{l}115(2 \times \text {, Beginn, vgr., und } \\
\text { aүळ Nтштм })\end{array}$ & \\
\hline 4,21 & $\begin{array}{l}\text { 10, } 112\left(\mathbb{( T /} \text { und }^{\circ \circ}\right), 116(\div \\
\text { und } \odot)\end{array}$ & $19,115,116,124$ & \\
\hline 4,22 & & 115 & \\
\hline 4,23 & $\begin{array}{l}3 \text { (> bei ерє мречоүळот), } 112 \\
\text { (bei ерє пеіют, Фा und }{ }^{\circ} \text { ) }\end{array}$ & 112 (bei єрє пєєळт), 115 & \\
\hline 4,24 & & 115 & $\begin{array}{l}\text { Tau-Ligatur (m2, für } \tau \dot{\varepsilon}- \\
\lambda \text { os?, Perikopenende?): } 102\end{array}$ \\
\hline 4,25 & $3(>), 102,116(\div$ und $C)$ & $19,115,116,124$ & \\
\hline 4,26 & 116 & $19,115,116,124$ & \\
\hline 4,27 & 102 & 124 & \\
\hline 4,28 & $141(>)$ & 19 (vgr.), 115 & \\
\hline 4,29 & $141(>)$ & 112 & \\
\hline 4,30 & 116 & $19,115,116$ & \\
\hline
\end{tabular}




\begin{tabular}{|c|c|c|c|}
\hline & Paragraphenzeichen & Initialen & Nummerierungen / Varia \\
\hline 4,31 & $\begin{array}{l}9,10(\cdot \dot{*} \cdot \text { und }>, \mathrm{m} 2), 102,116 \\
(\dot{*}), 141(\cdot \dot{*})\end{array}$ & $\begin{array}{l}\text { 9, 10, 19, 102, } 112 \text { (vgr.), 115, } \\
116 \text { (vgr.), } 141 \text { (vgr.) }\end{array}$ & \\
\hline 4,32 & & 115 & \\
\hline 4,33 & $141(>)$ & & \\
\hline 4,34 & $141(>)$ & $19,112,115$ & \\
\hline 4,35 & $\begin{array}{l}141(2 \times, \text {, , Beginn und eıc } \\
\text { 2ннте) }\end{array}$ & $\begin{array}{l}19 \text { (bei єıс гннте), } 112 \text { (bei } \\
\text { єı гннте), } 115 \text { ( } 2 \times \text {, Beginn } \\
\text { und єıс гннте), 141, } 190 \text { (bei } \\
\text { єıс гннте, vgr.) }\end{array}$ & 粶: 116 (Kreuz) \\
\hline 4,36 & 116 & $\begin{array}{l}112 \text { (bei aүw nycoore), 115, } \\
116 \text { ( } 2 \times \text {, Beginn und xekac } \\
\text { петхо) }\end{array}$ & $\begin{array}{l}\text { Markierung: } 116 \text { (kreuzför- } \\
\text { mig angeordnete Punkte } \\
\text { am Beginn und bei xєкac } \\
\text { пєтхо) }\end{array}$ \\
\hline 4,37 & $141(>)$ & 19,115 & \\
\hline 4,38 & $141(>), 154(\cdot \dot{x} \cdot)$ & $115,141,154$ & \\
\hline 4,39 & 141 (> mit $\dot{*})$ & 115,141 & \\
\hline 4,40 & $116(\div$ und $\odot)$ & $9,19,115,116$ & \\
\hline \multicolumn{4}{|l|}{4,41} \\
\hline \multicolumn{4}{|l|}{4,42} \\
\hline 4,43 & $4(>), 10(\cdot \dot{*}), 116(\cdot \dot{*})$ & $\begin{array}{l}\text { 4, 9, } 10 \text { (vgr.), 103, } 116 \\
\text { (vgr.), 121, } 190 \text { (vgr.) }\end{array}$ & Abschnitt 9: 10, 116 \\
\hline \multicolumn{4}{|c|}{ - } \\
\hline 4,45 & & 103 & \\
\hline 4,46 & $\begin{array}{l}1,4(>), 5,10(\dot{*}), 103,116 \\
(\cdot \dot{*}), 117\end{array}$ & $\begin{array}{l}\text { 4, } 9 \text { (vgr.), 10, } 19 \text { (vgr.), } \\
\text { 103, } 116 \text { (vgr.), 117, 121, } 190 \\
\text { (vgr.) }\end{array}$ & $\begin{array}{l}\text { Keph. 5: 9, }\{10 \text { (rezent, „5“, } \\
\text { nicht } \bar{\epsilon})\} \text {; Glosse: } 103 \text { (verti- } \\
\text { kal Buchstaben: c т ọ €̣) }\end{array}$ \\
\hline \multicolumn{4}{|l|}{4,47} \\
\hline 4,48 & & 19,103 & \\
\hline 4,49 & & 19 & \\
\hline \multicolumn{4}{|l|}{4,50} \\
\hline $\begin{array}{l}4,51 \\
4,52\end{array}$ & $116(\div$ und $\odot)$ & 116,117 & \\
\hline 4,52 & 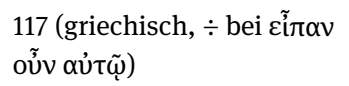 & 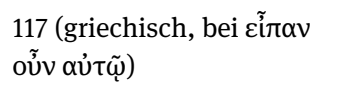 & \\
\hline \multicolumn{4}{|l|}{4,54} \\
\hline 5,1 & $\begin{array}{l}4(>), 5(>/ \odot), 10(\cdot \dot{*}), 12(\cdot \dot{*}), \\
116(\cdot \dot{*})\end{array}$ & $\begin{array}{l}\text { 1, 4, 5, 9, } 10 \text { (vgr.), 12, 19, } \\
116 \text { (vgr.), } 121\end{array}$ & $\begin{array}{l}\text { Keph. 6: 9; Abschnitt 10: } \\
\text { 116; Abschnitt 11: } 12\end{array}$ \\
\hline \multicolumn{4}{|c|}{ 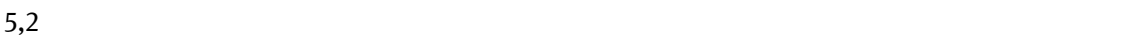 } \\
\hline \multicolumn{4}{|l|}{5,3} \\
\hline \multicolumn{4}{|l|}{5,4} \\
\hline 5,5 & 101 & 19,101 & \\
\hline \multicolumn{4}{|l|}{5,6} \\
\hline 5,7 & & 19 (bei госом аNок) & \\
\hline 5,8 & & 19 & \\
\hline
\end{tabular}




\begin{tabular}{|c|c|c|c|}
\hline & Paragraphenzeichen & Initialen & Nummerierungen / Varia \\
\hline \multicolumn{4}{|l|}{5,9} \\
\hline 5,10 & $* 116$ & 19, 103, 116 & \\
\hline \multicolumn{4}{|l|}{5,11} \\
\hline 5,12 & & 19 & \\
\hline 5,13 & & 19 (bei $\overline{\imath c}$ гар медчсегтч) & \\
\hline 5,14 & & 19, 246 (bei мunncd) & \\
\hline 5,15 & & 19 & \\
\hline \multicolumn{4}{|l|}{5,16} \\
\hline 5,17 & & 9 (vgr.), 19, 105 (vgr.) & $\begin{array}{l}\text { Markierung: } 116 \text { (kreuzför- } \\
\text { mig angeordnete Punkte) }\end{array}$ \\
\hline \multicolumn{4}{|l|}{5,18} \\
\hline 5,19 & $\begin{array}{l}\text { pbo, } 10(\dot{*}), * 12(\dot{*}), 116 \\
(\dot{*}), 121\end{array}$ & $\begin{array}{l}9 \text { (vgr.), } 10 \text { (vgr.), } 19 \text { (vgr.), } \\
105 \text { (vgr.), } 116 \text { (vgr.), 121, } \\
136\end{array}$ & $\begin{array}{l}\text { „Amen-Wort“ 5: 4; Ab- } \\
\text { schnitt 11: *10 (ז’ạ), } 116\end{array}$ \\
\hline \multicolumn{4}{|l|}{5,20} \\
\hline 5,21 & & 9 (vgr.), 19 & $\begin{array}{l}\text { Markierung (?): } 116 \text { (kreuz- } \\
\text { förmig angeordnete Punkte } \\
\text { rechts des Textes) }\end{array}$ \\
\hline
\end{tabular}

5,27

5,28

5,29

5,30

5,31

$116(\div$ und $\odot)$

12, 136, 167

12

19, 116, 167

$10(\stackrel{*}{*}), * 12(※$, Position hier 10 (vgr.), *116 (vgr.), 167

Abschnitt 12: 10 (m2), 116 unsicher), $* 116(\dot{*})$

(m2), 167

5,32

19

116

19,167

19

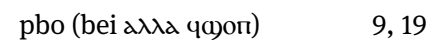




\begin{tabular}{|c|c|c|c|}
\hline & Paragraphenzeichen & Initialen & Nummerierungen / Varia \\
\hline \multicolumn{4}{|l|}{5,47} \\
\hline 6,1 & $\begin{array}{l}\text { pbo, } 1(>), 3(>), 5(>/ \odot), 116 \\
(\stackrel{\times}{*})\end{array}$ & $\begin{array}{l}\text { 5, } 9 \text { (vgr.), } 10 \text { (vgr.), } 19 \\
\text { (vgr.), } 116 \text { (vgr.), 121, 139, } \\
167\end{array}$ & $\begin{array}{l}\text { Keph. 7: 9; Abschnitt 13: } \\
\text { 116, } 139 \text { (m2), } 167\end{array}$ \\
\hline \multicolumn{4}{|c|}{ 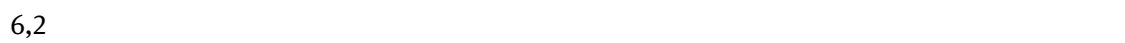 } \\
\hline 6,3 & $4(>)$ & 4,19 & \\
\hline \multicolumn{4}{|l|}{6,4} \\
\hline 6,5 & & 19,116 & \\
\hline \multicolumn{4}{|l|}{6,6} \\
\hline 6,7 & pbo, 116 & $19,116,139$ & \\
\hline 6,8 & 116 & 19,139 & \\
\hline \multicolumn{4}{|l|}{6,9} \\
\hline 6,10 & 116 & 116,139 & \\
\hline 6,11 & & 19,139 & \\
\hline \multicolumn{4}{|l|}{6,12} \\
\hline \multicolumn{4}{|l|}{6,13} \\
\hline \multicolumn{4}{|l|}{6,14} \\
\hline 6,15 & 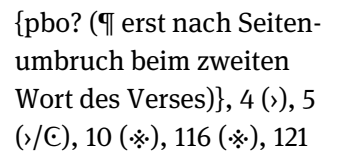 & $\begin{array}{l}\text { 4, 5, } 9 \text { (vgr.), } 10 \text { (vgr.), } 19 \\
\text { (vgr.), 103, } 116 \text { (vgr.), } 121\end{array}$ & $\begin{array}{l}\text { \{Keph. 8: } 9 \text { (am Text nicht } \\
\text { bezeichnet)\}; \{Keph. 9: } 10 \\
\text { (rezent)\}; Abschnitt 14: } 10 \\
(\mathrm{~m} 2), * 116(\mathrm{~m} 2)\end{array}$ \\
\hline 6,16 & & 103 & \\
\hline \multicolumn{4}{|l|}{6,17} \\
\hline 6,18 & & 103 & \\
\hline \multicolumn{4}{|l|}{6,19} \\
\hline 6,20 & & 103 & \\
\hline \multicolumn{4}{|l|}{6,21} \\
\hline 6,22 & $116(\dot{x})$ & 9, 19, 103, 116 (vgr.) & $\dagger: 116$ (Kreuz, cf. 6,23) \\
\hline 6,23 & & 103 & 焘: 116 (Kreuz, cf. 6,22) \\
\hline 6,24 & $10(\dot{x}), 116(\dot{x})$ & 10 (vgr), 19, 116 (vgr.) & $\begin{array}{l}\text { Abschnitt 15: } 10(\mathrm{~m} 2), 116 \\
(\mathrm{~m} 2)\end{array}$ \\
\hline \multicolumn{4}{|l|}{6,25} \\
\hline 6,26 & pbo, $116(\div$ und $\odot), 121$ & 103,116 & „Amen-Wort“ 8: 4 \\
\hline 6,27 & $\begin{array}{l}112\left(\mathbb{T} \text { und }{ }^{\circ} \text { bei } \alpha \lambda \lambda d \text { Tє- }\right. \\
2 \mathrm{P}), 116(\div \text { und } \odot)\end{array}$ & $\begin{array}{l}\text { 9, } 19 \text { (vgr.), 103, 112, } 116 \\
\text { (vgr.) }\end{array}$ & \\
\hline 6,28 & $112\left(\right.$ (ा| und $\left.^{\circ \circ}\right), 116(\div$ und $\odot)$ & $103,112,116,117$ & \\
\hline 6,29 & pbo, $116(\div$ und $\odot)$ & $103,116,117$ & \\
\hline 6,30 & $112\left(\right.$ (ा und $\left.^{\circ \circ}\right), 141(>)$ & $103,112,117$ & \\
\hline 6,31 & $103(\dot{*}), 141(>)$ & 103 & \\
\hline 6,32 & 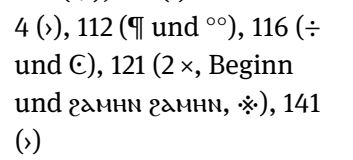 & $\begin{array}{l}\text { 4, 9, } 19,103,112,116,117, \\
121 \text { ( } 2 \times, \text { Beginn und гамнм } \\
\text { 2амнм), } 141 \text { (vgr.) }\end{array}$ & „Amen-Wort“ 9: 4 \\
\hline 6,33 & $112\left(\mathbb{T}\right.$ und $\left.^{\circ \circ}\right)$ & 103,112 & \\
\hline 6,34 & $112\left(\right.$ (ा| und $\left.^{\circ \circ}\right), 116(\div$ und $\odot)$ & $9,19,112,116,117, * 141$ & \\
\hline
\end{tabular}




\begin{tabular}{|c|c|c|c|}
\hline & Paragraphenzeichen & Initialen & Nummerierungen / Varia \\
\hline 6,35 & $112\left(\right.$ (I und $\left.^{\circ \circ}\right)$ & 112,117 & \\
\hline 6,36 & $116(\div$ und $\odot)$ & 19 (bеi мтетмпістеү), 116 & \\
\hline 6,37 & $\begin{array}{l}103(\dot{*}), 112 \text { (बा und }{ }^{\circ} \text { bei } \\
\text { пєТNHY (d)גPol) }\end{array}$ & $\begin{array}{l}* 19,103,112 \text { (bei пєтNнү } \\
\text { (a)גPol) }\end{array}$ & \\
\hline \multicolumn{4}{|l|}{6,38} \\
\hline 6,39 & $116(\div$ und $\odot)$ & 19,116 & \\
\hline 6,40 & $112\left(\mathbb{\text { II }}\right.$ und $\left.^{\circ \circ}\right), 116(\div$ und $\odot)$ & 19, 112, 116, 155 (vgr.) & \\
\hline 6,41 & $\{\mathrm{pbo}\}, 5(>/ \odot), 116$ & 5, 19, 103, 112 (vgr.), 116, 117 & \\
\hline 6,42 & $\begin{array}{l}112\left(\mathbb{T} \text { und }^{\circ \circ} \text { ), } 116 \text { (bei } x \in\right. \\
\text { ayх00c } x \epsilon)\end{array}$ & 112, 116, 155 (vgr.) & \\
\hline 6,43 & $\begin{array}{l}\text { pbo, } 112\left(2 \times \text {, } \text { II und }{ }^{\circ} \text { bei }\right. \\
\text { Beginn, } \div \text { bei oyon NIm }), 116 \\
(\div \text { und } \odot)\end{array}$ & $\begin{array}{l}\text { 19, 103, } 112(2 \times \text {, Beginn } \\
\text { und oүon NIM), 116, } 117\end{array}$ & \\
\hline \multicolumn{4}{|c|}{. } \\
\hline 6,45 & & $\begin{array}{l}\text { 103, 112, } 155(2 \times, \text { Beginn } \\
\text { und oүом мाм Nтачсютм })\end{array}$ & Zitat $\rightarrow »: 4,10$ \\
\hline 6,46 & & 103 & \\
\hline 6,47 & $\begin{array}{l}\{\mathrm{pbo}\}, 5(>/ \subset), 116(\dot{*}), 121 \\
(\dot{*})\end{array}$ & $\begin{array}{l}\text { 5, } 9 \text { (vgr.), 10, } 19 \text { (vgr.), 103, } \\
116 \text { (vgr.), } 121\end{array}$ & $\begin{array}{l}\text { „Amen-Wort“ 10: 4; Ab- } \\
\text { schnitt 16: } 10 \text { (m2), } 116 \text { (m2) }\end{array}$ \\
\hline \multicolumn{4}{|c|}{ 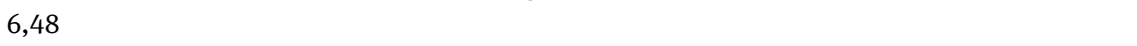 } \\
\hline \multicolumn{4}{|l|}{6,49} \\
\hline \multicolumn{4}{|l|}{6,50} \\
\hline 6,51 & & 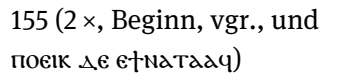 & \\
\hline 6,52 & $116(\div$ und $\odot)$ & $19,103,116,117$ & \\
\hline 6,53 & $116(\div$ und $\odot), 121$ & $19,116,117,121$ & „Amen-Wort“ 11: 4 \\
\hline \multicolumn{4}{|l|}{6,54} \\
\hline \multicolumn{4}{|l|}{6,55} \\
\hline 6,56 & & & $\begin{array}{l}\$ \text { (?, cf. auch 6,62): } 116 \text { (bei } \\
\text { aүळ єтсо мпасмоч Kreuz } \\
\text { rechts der Kol.) }\end{array}$ \\
\hline \multicolumn{4}{|r|}{ 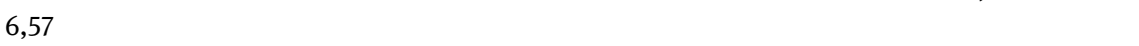 } \\
\hline \multicolumn{4}{|l|}{6,58} \\
\hline 6,59 & $\begin{array}{l}\text { pbo, 4(>/€), } 10(\cdot \dot{*} \text { von } \mathrm{m} 2, \\
\text { unfertig), } 116(\cdot \dot{*})\end{array}$ & $\begin{array}{l}4 \text { (vgr.), } 9 \text { (vgr.), 19, 103, } \\
116 \text { (vgr.) }\end{array}$ & \\
\hline 6,60 & & 117 (griechisch) & \\
\hline 6,61 & 116 & 19 (vgr.), 116, 117 (griechisch) & \\
\hline 6,62 & & 103 & $\begin{array}{l}\text { Schlussmarkierung (?, cf. } \\
\text { auch 6,56): } 116 \text { (vor dem } \\
\text { Vers ein Andreaskreuz) }\end{array}$ \\
\hline \multicolumn{4}{|r|}{ (1) } \\
\hline \multicolumn{4}{|l|}{6,64} \\
\hline \multicolumn{4}{|l|}{6,65} \\
\hline \multicolumn{4}{|l|}{6,66} \\
\hline 6,67 & $116(\div$ und $\odot)$ & 19, 105, 116, 177 (griechisch) & \\
\hline 6,68 & & 19,139 & \\
\hline
\end{tabular}




\begin{tabular}{|c|c|c|c|}
\hline & Paragraphenzeichen & Initialen & Nummerierungen / Varia \\
\hline \multicolumn{4}{|l|}{6,69} \\
\hline 6,70 & pbo, $10(\dot{*}), 116(\dot{*})$ & $\begin{array}{l}9 \text { (vgr.), } 10 \text { (vgr.), } 19 \text { (vgr.), } \\
105,116 \text { (vgr.), 117, 136, } 139\end{array}$ & $\begin{array}{l}\text { Abschnitt 17: } 10(\mathrm{~m} 2), 116 \\
(\mathrm{~m} 2), 139(\mathrm{~m} 2)\end{array}$ \\
\hline \multicolumn{4}{|c|}{ - } \\
\hline 7,1 & $\begin{array}{l}4(>), 5(>/ \odot), 116(\div \text { und } * \dot{*}) \\
117,162\end{array}$ & $\begin{array}{l}\text { 4, 5, 9, 19, 103, } 116 \text { (vgr.), } \\
117,121, * 136,139\end{array}$ & $\begin{array}{l}\text { Glosse: } 103 \text { ([ - ] гоo[Y] eT- } \\
\text { оүдав) }\end{array}$ \\
\hline 7,2 & & $9,19,117,139$ & \\
\hline 7,3 & 116 & 116 & \\
\hline 7,4 & $\begin{array}{l}141 \text { (> bei ec)xe keıpe, Vers- } \\
\text { beginn verloren) }\end{array}$ & 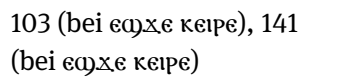 & \\
\hline \multicolumn{4}{|c|}{ 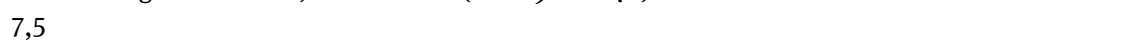 } \\
\hline 7,6 & $116(\div$ und $\odot), 141(\curvearrowright)$ & $116,117,139,141$ & \\
\hline \multicolumn{4}{|c|}{ (.) } \\
\hline 7,8 & $141(>)$ & $103,139,141$ & \\
\hline 7,9 & $116(\div$ und $\odot), 117,141(>)$ & $\begin{array}{l}9 \text { (vgr.), 19, 103, 116, 117, } \\
141\end{array}$ & \\
\hline 7,10 & pbo & 136 & \\
\hline 7,11 & $141(>)$ & 117,141 & \\
\hline 7,12 & 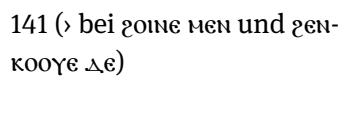 & 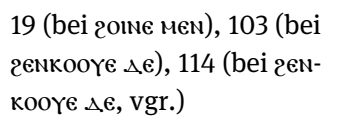 & \\
\hline 7,13 & $141(>), 162$ & & \\
\hline 7,14 & $4(>), 9,116,121,141(\dot{*}), 162$ & $\begin{array}{l}\text { 4, } 9 \text { (vgr.), 10, 19, 103, } 116 \\
\text { (vgr.), 117, 121, 123, } 141 \\
\text { (vgr.), } 285 \text { (vgr.) }\end{array}$ & 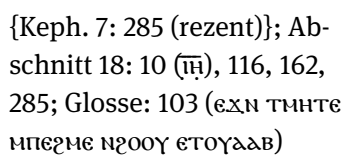 \\
\hline
\end{tabular}

7,15

7,16

pbo, $141($ ()

19, 117, 285

7,17

7,18

103

7,19

162

234

7,20 pbo

$19,117,234$

7,21 pbo, $116(\div$ und $\odot)$

9 (vgr.), 19, 101, 116, 117,

234

7,22

19

7,23

7,24

7,25

116

123 (bei ммєчвсл)

7,26

7,27

9, 19, 116, 117 (griechisch)

115 (bei мнпюс аүєіме)

115

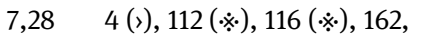

9 (vgr.), 19 (vgr.), 112 (vgr.), Glosse: 234 (ехN өגн, am

270 (Kleeblattkreuz)

115, 116 (vgr.), 117, 234

(vgr.), 270 (vgr.)

Unterrand, Zuweisung zu

Vers unsicher) 


\begin{tabular}{|c|c|c|c|}
\hline & Paragraphenzeichen & Initialen & Nummerierungen / Varia \\
\hline 7,30 & $112(\div), 116$ & $\begin{array}{l}19,112,115,116,117,250, \\
270\end{array}$ & \\
\hline 7,31 & $\begin{array}{l}1,112(2 \times \text {, Beginn und yNap } \\
\text { 2oүe, } \div \div), 116,125(>)\end{array}$ & $112,117,125,136$ & \\
\hline 7,32 & $116,125(\div), 270(\div)$ & $\begin{array}{l}9 \text { (vgr.), 19, 112, 115, 123, } 125 \\
\text { (vgr.), 134, 136, } 270 \text { (vgr.) }\end{array}$ & 米: $4(\div)$ \\
\hline 7,33 & 4, 116, $125(>), 250(>)$ & $\begin{array}{l}\text { 19, 112, 115, 117, } 125 \text { (vgr.), } \\
134,136,250\end{array}$ & \\
\hline \multicolumn{4}{|c|}{ - } \\
\hline 7,35 & $112\left(\mathbb{( I |}\right.$ und $\left.^{\circ \circ}\right)$ & $\begin{array}{l}\text { 112, } 115 \text { (bei anon NTnNąє), } \\
117,136\end{array}$ & \\
\hline 7,36 & & 115 & \\
\hline 7,37 & $\begin{array}{l}4(>), 5(>/ \odot), 10(\dot{*}), * 101 \\
(\cdot \dot{*}), 112(\dot{*}), 116(\cdot \dot{*}), 117, \\
125((\dot{*}), 162,234(\dot{*}), 250 \\
(\odot / \cdot \dot{*})\end{array}$ & $\begin{array}{l}\text { 4, 5, } 9 \text { (vgr.), } 10 \text { (vgr.), 19, } \\
* 101,112 \text { (vgr.), } 115 \text { (vgr.), } \\
116 \text { (vgr.), 117, 121, } 125 \\
\text { (vgr.), } 136 \text { (vgr.), } 234 \text { (vgr.), } \\
250\end{array}$ & $\begin{array}{l}\text { Abschnitt 19: } 10 \text { (m2), }\{115 \\
\text { (m2, Zahl fehlt tatsäch- } \\
\text { lich)\}, } 116(\mathrm{~m} 2), 125(\mathrm{~m} 2), \\
\text { 162, \{250\}; Glosse: } 234 \text { (exN } \\
\text { өגн; wohl zu diesem Vers) }\end{array}$ \\
\hline 7,38 & & & Zitat $\rightarrow »: 4$ \\
\hline 7,39 & & $\begin{array}{l}\text { 19, 115, } 136 \text { (bеi мем̄па тоүхı } \\
\overline{\Pi N \lambda} \text {, vgr.) }\end{array}$ & \\
\hline 7,40 & $112(\div)$ & $\begin{array}{l}\text { 19, 112, } 115 \text { (vgr.), 117, 134, } \\
234\end{array}$ & \\
\hline 7,41 & $112(\div)$ & $\begin{array}{l}\text { 112, } 115(2 \times \text {, Beginn und } \\
\text { 2olve), } 134 \text { (bei golve) }\end{array}$ & \\
\hline 7,42 & $112(\bigodot)$ & 112,115 & Zitat $\rightarrow »: 4$ \\
\hline 7,43 & 112 (पा und $\left.{ }^{\circ}{ }^{\circ}\right), 116$ & $\begin{array}{l}19,103,105,112,115,116, \\
117,134,136\end{array}$ & \\
\hline 7,44 & & 112,115 & \\
\hline 7,45 & $\begin{array}{l}112\left(2 \times, \text { Beginn } \text { Iा und }{ }^{\circ \circ}\right. \\
\text { und nexe NH } \div), 125 \text { (> und } \\
\div), 250\end{array}$ & $\begin{array}{l}\text { 105, } 112 \text { ( } 2 \times \text {, Beginn und } \\
\text { пєхє NH), 115, 117, } 125 \\
\text { (vgr.), 136, } 159 \text { (vgr.), 197, } \\
250\end{array}$ & \\
\hline 7,46 & $\begin{array}{l}10(\dot{*}), 112(\div), 116(\dot{*}), 125 \\
(\dot{*})\end{array}$ & $\begin{array}{l}10 \text { (vgr.), } 19 \text { (vgr.), 103, 112, } \\
116 \text { (vgr.), 117, } 125\end{array}$ & $\begin{array}{l}\text { Abschnitt 20: } 10(\mathrm{~m} 2), 116 \\
(\mathrm{~m} 2), 125(\mathrm{~m} 2), 139(\mathrm{~m} 2)\end{array}$ \\
\hline 7,47 & pbo, $116(\div$ und $\odot)$ & $103,116,117,139,250$ & \\
\hline 7,48 & & 103 & \\
\hline \multicolumn{4}{|l|}{7,49} \\
\hline 7,50 & $112\left(\right.$ (ा und $\left.^{\circ \circ}\right), 116,125$ ( >) & $\begin{array}{l}\text { 9, 19, 103, 105, 112, 116, 117, } \\
125 \text { (vgr.), 134, 139, } 197\end{array}$ & \\
\hline \multicolumn{4}{|l|}{7,51} \\
\hline 7,52 & *210 (※. unsicher $)$ & $\begin{array}{l}\text { 19, } 103 \text { (bei готгт мNегрд- } \\
\phi н), 112,116 \text { (bei гот२т nме- } \\
\text { грдфн), 117, 136, } 139\end{array}$ & \\
\hline
\end{tabular}


7,53-8,11 sind in Manuskripten auf Sahidisch, Lykopolitanisch und Proto-Bohairisch bisher nicht belegt. Für einen wahrscheinlichen sahidischen Textzeugen auf einem Kalksteinostrakon siehe 2.1. Pericope adulterae.

\begin{tabular}{|c|c|c|c|}
\hline & Paragraphenzeichen & Initialen & Nummerierungen / Varia \\
\hline 8,12 & 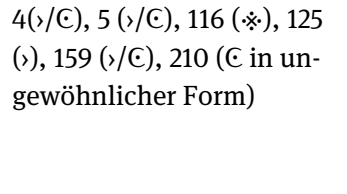 & 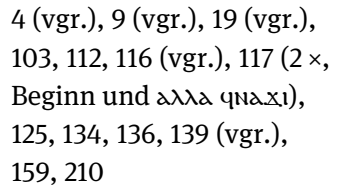 & Glosse: 103 (кг еXN пе $\overline{\mathrm{XC}})$ \\
\hline 8,13 & $112(\div), 116(\div$ und $\odot)$ & $\begin{array}{l}\text { 103, 105, 112, 117, 134, } 136 \\
\text { (vgr.) }\end{array}$ & \\
\hline 8,14 & $\begin{array}{l}112\left(\text { (II und }{ }^{\circ}\right), 116(\div \text { und } \\
\odot), * 159(* \odot)\end{array}$ & 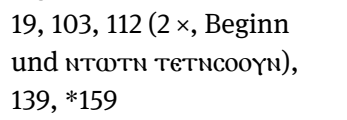 & \\
\hline 8,15 & & 103 & \\
\hline 8,16 & & & \\
\hline 8,17 & $\left\{\right.$ pbo\}, $112\left(\mathbb{( T}\right.$ und $\left.^{\circ \circ}\right), 210(>)$ & 19, 103, 112, 136 (vgr.), 210 & $\begin{array}{l}\text { Zitat } \rightarrow »: 1,4 ; \ddagger: 116 \\
\text { (Punkte) }\end{array}$ \\
\hline 8,18 & & & \\
\hline 8,19 & 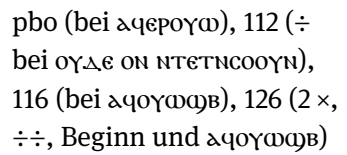 & 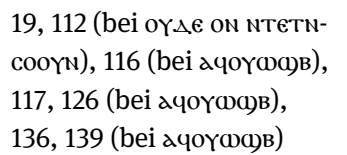 & \\
\hline 8,20 & $112(\dot{*}), 125(\dot{*}), 126(\div)$ & $\begin{array}{l}9 \text { (vgr.), 19, 103, } 112 \text { (vgr.), } \\
117,125 \text { (vgr.), 126, 136, 139, } \\
285\end{array}$ & $\begin{array}{l}\{\text { Keph. 8: } 285 \text { (rezent)\}; can. } \\
\text { 88/1: } 285\end{array}$ \\
\hline 8,21 & $\begin{array}{l}4(>), 116(\div \text { und } \odot), 125(>), \\
159(\odot)\end{array}$ & $\begin{array}{l}\text { 4, 103, 112, 116, 117, 125, } 126 \\
\text { (vgr.), } 136 \text { (bei дүс пмд } \\
\text { дмок), 159, } 285\end{array}$ & can. 89/X: 285 \\
\hline 8,22 & $112\left(\boldsymbol{\Phi}\right.$ und $\left.^{\circ \circ}\right)$ & $19,103,112,117,285$ & \\
\hline 8,23 & $112\left(\boldsymbol{\Phi}\right.$ und $\left.^{\circ \circ}\right), 116$ & $\begin{array}{l}103 \text { (bеi мтютм мтетм), 112, } \\
116,117,126,285\end{array}$ & \\
\hline 8,24 & & 136 & \\
\hline 8,25 & 125 (> bei nexe $\overline{\mathrm{Ic}})$ & 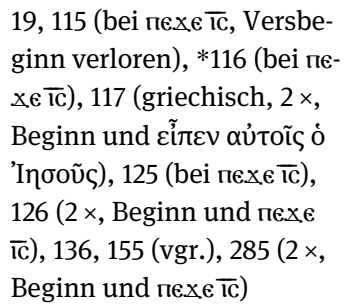 & \\
\hline \multicolumn{4}{|l|}{8,26} \\
\hline 8,27 & & 115, 117 (griechisch) & \\
\hline
\end{tabular}




\begin{tabular}{|c|c|c|c|}
\hline & Paragraphenzeichen & Initialen & Nummerierungen / Varia \\
\hline 8,28 & 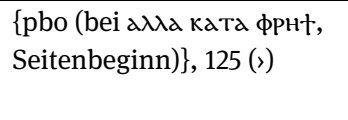 & $\begin{array}{l}\text { 19, 105, 115, 116, } 117 \text { (grie- } \\
\text { chisch), 125, } 155 \text { (vgr.), } 285\end{array}$ & $\begin{array}{l}\text { *(?): } 116 \text { (Kreuz unter der } \\
\text { Kol.); \{Keph. 8: } 285 \text { (re- } \\
\text { zent)\} }\end{array}$ \\
\hline 8,29 & & 115,136 & \\
\hline 8,30 & $\begin{array}{l}10(\dot{*}), 116(\dot{*}), 125(\div \text { und } \\
\dot{*}), 285(\dot{*})\end{array}$ & $\begin{array}{l}9 \text { (vgr.), } 10 \text { (vgr), 115, } 116 \\
\text { (vgr.), } 117 \text { (griechisch), } 125 \\
\text { (vgr.), } 285\end{array}$ & $\begin{array}{l}\text { Abschnitt 21: } 10 \text { (m2), } 116 \\
(\mathrm{~m} 2), 125(\mathrm{~m} 2), 155(\mathrm{~m} 2), 285\end{array}$ \\
\hline 8,31 & $\begin{array}{l}126(\odot \text { (einzige } \odot \text { in } J) \text { und } \\
\div)\end{array}$ & $\begin{array}{l}9 \text { (vgr.), 19, 105, } 115 \text { (vgr.), } \\
117 \text { (griechisch), } 155 \text { (vgr.), } \\
285\end{array}$ & Abschnitt 21: 115 (m2) \\
\hline 8,32 & & 136 & \\
\hline 8,33 & pbo, $126(\div)$ & $\begin{array}{l}\text { 19, } 105 \text { (vgr.), 115, 136, } 155 \\
\text { (vgr.), } 285\end{array}$ & \\
\hline 8,34 & $\begin{array}{l}\text { pbo, } 116(\div \text { und } \odot), 125(>) \text {, } \\
126(\div), 238(\div)\end{array}$ & $\begin{array}{l}\text { 9, } 19 \text { (vgr.), } 105 \text { (vgr.), 115, } \\
\text { 116, 123, 125, 126, } 136 \text { (bei } \\
\text { 2амнм әамнм), } 155 \text { (vgr.), } \\
238\end{array}$ & „Amen-Wort““ 12: 4 \\
\hline 8,35 & $\begin{array}{l}\text { pbo (bei пю)нрі } \triangle € \text { мөоч), } \\
238(\div)\end{array}$ & 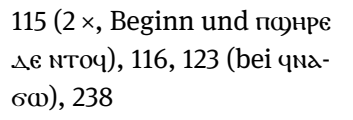 & \\
\hline 8,36 & $116(\div$ und $\odot)$ & 115,116 & \\
\hline 8,37 & $\begin{array}{l}\{\text { pbo\}, } 116(\dot{*}), 125(>), 238 \\
(\div)\end{array}$ & 116, 136, 136, 139 (vgr.), 238 & \\
\hline 8,38 & $141(>)$ & $\begin{array}{l}19 \text { (bei мтотм } 6 \text { ) }), 105,115 \\
(2 \times \text {, Beginn und Nтотм } 6 \epsilon) \\
\text { 136, } 155 \text { (vgr.) }\end{array}$ & \\
\hline 8,39 & $\begin{array}{l}\text { pbo, } 106(2 \times \text {, Beginn und } \\
\text { пєхє } \overline{\mathrm{ic}} \mathrm{Na \gamma}), 141(>), 238(\div)\end{array}$ & 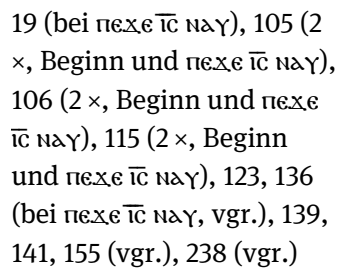 & \\
\hline 8,40 & $13,238(\div)$ & $\begin{array}{l}115 \text { (bei оүрФме, vgr.) } 136 \\
\text { (bei оүрФме), } 238\end{array}$ & \\
\hline 8,41 & 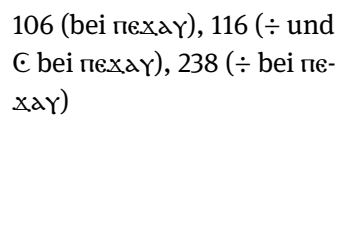 & 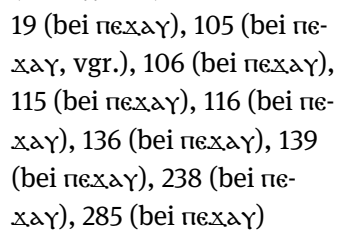 & \\
\hline
\end{tabular}




\begin{tabular}{|c|c|c|c|}
\hline & Paragraphenzeichen & Initialen & Nummerierungen / Varia \\
\hline 8,42 & $\begin{array}{l}\{\text { pbo\}, 106, } 116(\div \text { und } \odot) \\
238 \text { (> bei амок гар) }\end{array}$ & 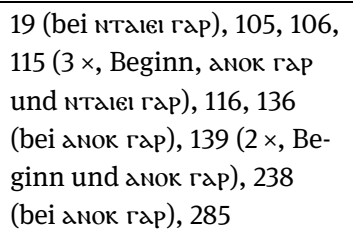 & $\{$ Keph. 8: 285 (rezent) $\}$ \\
\hline 8,43 & 106 & 106,115 & \\
\hline 8,44 & 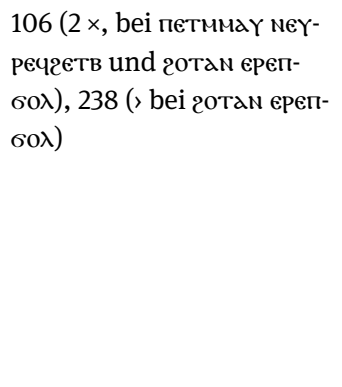 & 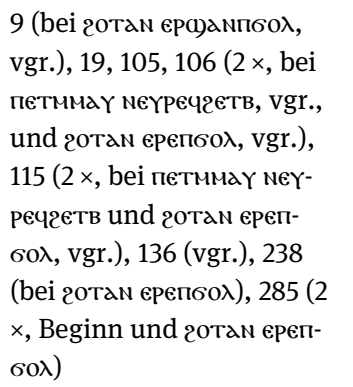 & \\
\hline 8,45 & 5,238 & $136,238,285$ & \\
\hline 8,46 & $106(\odot)$ & $19,106,136,139$ & \\
\hline 8,47 & & 285 & $\{$ Keph. 8: 285 (rezent) $\}$ \\
\hline 8,48 & 106 & 19, 105 (vgr.), 106, 139, 285 & \\
\hline 8,49 & pbo, 106 & $\begin{array}{l}19 \text { (bеi анок мм Аалмоміом), } \\
106,127,136,139\end{array}$ & \\
\hline 8,50 & 238 & 136,238 & \\
\hline 8,51 & $\begin{array}{l}\text { pbo (unsicher), } 4 \text { ( (), 105, } \\
\text { 106, } 125 \text { (২), } 238\end{array}$ & $\begin{array}{l}\text { 4, } 9 \text { (vgr.), 19, 105, 106, } 125 \\
\text { (vgr.), 127, } 136 \text { (vgr.), } 238 \\
\text { (vgr.), } 285\end{array}$ & „Amen-Wort“ 13: 4 \\
\hline 8,52 & $\begin{array}{l}\text { 106, } 238 \text { ( } 2 \times \text {, Beginn und } \\
\text { дврдәдм дчмоү) }\end{array}$ & 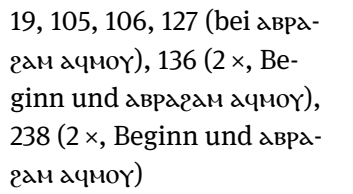 & \\
\hline 8,53 & 238 & 19,238 & \\
\hline 8,54 & pbo, 106, 238 & $\begin{array}{l}\text { 19, } 105 \text { (vgr.), 106, } 125 \\
\text { (ohne } \odot), 127,238,285\end{array}$ & $\{$ Keph. 8: 285 (rezent) $\}$ \\
\hline 8,55 & & 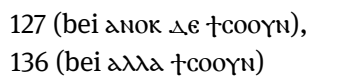 & \\
\hline 8,56 & $\begin{array}{l}\text { pbo (unsicher), } 4(>), 106 \text {, } \\
109(\div), 125(\dot{*})\end{array}$ & $\begin{array}{l}4 \text { (vgr.), } 19 \text { (vgr.), 106, } 125 \\
\text { (vgr.), 127, } 136 \text { (vgr.), } 285\end{array}$ & \\
\hline 8,57 & 109 & 105,106 & \\
\hline 8,58 & & $19,105,106,127,285$ & „Amen-Wort“ 14: 4 \\
\hline 8,59 & $106(\odot)$ & $\begin{array}{l}\text { 106, } 136 \text { (tatsächlich letztes } \\
\text { Wort von 8,58) }\end{array}$ & \\
\hline
\end{tabular}




\begin{tabular}{|c|c|c|c|}
\hline & Paragraphenzeichen & Initialen & Nummerierungen / Varia \\
\hline 9,1 & $\begin{array}{l}\text { 1, 4(২/৫), 5, } 10(\dot{*}), 12(\dot{*}), \\
\text { 105, 106, } 109(\dot{*}), 125(\dot{*}), \\
127(\dot{*})\end{array}$ & $\begin{array}{l}4 \text { (vgr.), } 9 \text { (vgr.), } 10 \text { (vgr.), } \\
\text { 12, } 19 \text { (vgr.), } 105 \text { (vgr.), 106, } \\
109 \text { (vgr.), } 125 \text { (vgr.), 127, } \\
136 \text { (vgr.), } 141 \text { (vgr.), } 285 \\
\text { (vgr.) }\end{array}$ & $\begin{array}{l}\text { Keph. 9: 9, }\{285 \text { (rezent)\}; } \\
\text { Keph. 10: } 285(*) \text {; Abschnitt } \\
\text { 22: } 10(\mathrm{~m} 2), 12(\mathrm{~m} 2 \text {, rechts } \\
\text { der Kol.), } 109(\mathrm{~m} 2), 125,285\end{array}$ \\
\hline \multicolumn{4}{|l|}{9,2} \\
\hline 9,3 & pbo, 106 & $19,105,106,285$ & \\
\hline 9,4 & pbo & $\begin{array}{l}\text { 9, } 19 \text { (bеi смнү мбІ теү()н), } \\
136 \text { (bei смнү мбІ теү()н, } \\
\text { vgr.), } 285\end{array}$ & \\
\hline 9,5 & 106 & 106 & \\
\hline 9,6 & $109(\div)$ & $\begin{array}{l}105 \text { (vgr.), } 136 \text { (vgr.), 174, } \\
285\end{array}$ & \\
\hline 9,7 & 106 & 106, 136 (bеi дчвюк бє) & \\
\hline 9,8 & 106 & $105,106,285$ & $\{$ Keph. 9: 285 (rezent)\} \\
\hline 9,9 & $\begin{array}{l}106(2 \times, \text { Beginn und NTOч } \\
\Delta \in \text { Nєч } \times \text { ( ) }\end{array}$ & 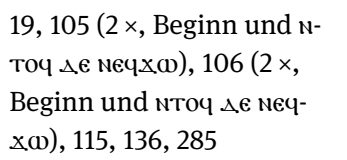 & \\
\hline 9,10 & & 105 (vgr.), 115, 136, 174, 285 & \\
\hline 9,11 & $\begin{array}{l}\text { pbo, } 106(2 \times \text {, Beginn und } \\
\text { аүш пехач) }\end{array}$ & $\begin{array}{l}\text { 19, } 103 \text { (bei alвळк } 6 \epsilon), 106 \\
(2 \times, \text { Beginn und aүळ пє- } \\
\text { Хач), } 115\end{array}$ & \\
\hline 9,12 & & 106, 115, 125, 136 (vgr.) & \\
\hline 9,13 & $116(\dot{*}), 125(>)$ & $\begin{array}{l}19 \text { (vgr.), } 105 \text { (vgr.), } 116 \\
\text { (vgr.), 125, } 285\end{array}$ & \\
\hline \multicolumn{4}{|l|}{9,14} \\
\hline 9,15 & $106,109(\div)$ & $\begin{array}{l}19(\text { bei nточ } \triangle \epsilon), 103,105 \\
106(2 \times, \text { Beginn und NToч } \\
\Delta \epsilon), 115\left(2 \times, \text { Beginn und } \text { N- }^{-}\right. \\
\text {точ } \triangle \epsilon), 136,173,174,285\end{array}$ & \\
\hline 9,16 & 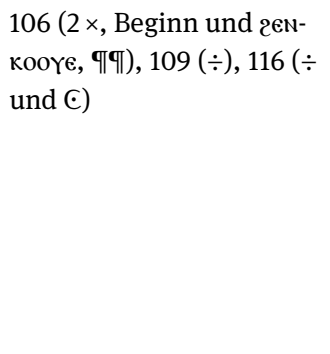 & 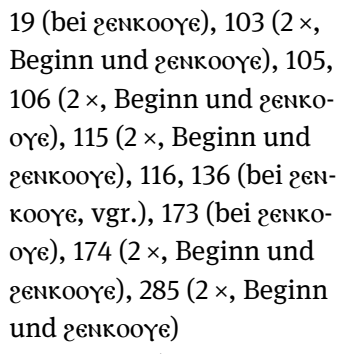 & \{Keph. 9: 285 (rezent)\} \\
\hline 9,17 & $\begin{array}{l}106(2 \times \text {, Beginn und nточ } \\
\triangle \epsilon), 109(\div \div, 2 \times \text {, Beginn } \\
\text { und мточ } \triangle \epsilon)\end{array}$ & 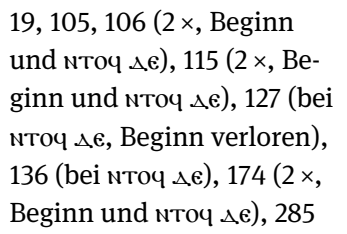 & \\
\hline
\end{tabular}




\begin{tabular}{|c|c|c|c|}
\hline & Paragraphenzeichen & Initialen & Nummerierungen / Varia \\
\hline 9,18 & $127(\div)$ & 105 (vgr.), 285 & \\
\hline 9,19 & 106 & 106,136 & \\
\hline 9,20 & $\begin{array}{l}\text { pbo, } 106,116(\div \text { und } \odot), 127 \\
(\div \text { bei Twcooүм хє пал пє) }\end{array}$ & $\begin{array}{l}19 \text { (vgr.), } 102 \text { (bei чмау єво } \\
\text { in 9,19, = Zeilenbeginn), } \\
\text { 103, 105, 106, 115, 116, 127, } \\
\text { 134, } 136 \text { (vgr.), 174, } 285\end{array}$ & \\
\hline 9,21 & & 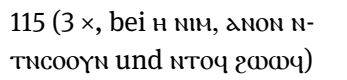 & \\
\hline 9,22 & 106, 127 ( $\div$ bеi меүрготе) & $\begin{array}{l}103 \text { (веі гомодоге ммоч), } \\
\text { 105, 106, } 115 \text { (bеi медүоүс), } \\
\text { 127, } 134 \text { ( } 2 \times \text {, Beginn und } \\
\text { медүоү(), } 136 \text { ( } 2 \times \text {, Beginn } \\
\text { und медүоү(), 174, } 285\end{array}$ & $\{$ Keph. 9: 285 (rezent) $\}$ \\
\hline 9,23 & & 115 & \\
\hline 9,24 & $\begin{array}{l}102(\div), 106,116(\div \text { und } \odot) \text {, } \\
127(\div \div, 2 \times \text {, Beginn und } \\
\text { Ende })\end{array}$ & $\begin{array}{l}\text { 19, 103, } 105 \text { (vgr.), 106, 108, } \\
\text { 115, 116, 127, 134, 136, 174, } \\
285\end{array}$ & $\begin{array}{l}*: 102(\text { Ini. mit } * \text {. siehe } \\
\text { auch 9,38); } 127(\div)\end{array}$ \\
\hline 9,25 & pbo, $12,106,116$ & $\begin{array}{l}12,103,105,106,108,115, \\
116,127,174,285\end{array}$ & \\
\hline 9,26 & 106, $116(\div$ und $\odot), 218(\odot)$ & $\begin{array}{l}106,108,115,116,127,134, \\
218,285\end{array}$ & \\
\hline 9,27 & $\begin{array}{l}\text { pbo, } 106(\odot), 127(\div), * 134 \\
\text { (einzige } * \text {, Ini. verloren) }\end{array}$ & $\begin{array}{l}\text { 19, } 103 \text { (bei oүon пететN- } \\
\text { oүe()), 105, 106, 108, 127, } \\
134,136,174,285\end{array}$ & \\
\hline 9,28 & 106 & $\begin{array}{l}\text { 105, 106, 136, } 142 \text { (bei anon } \\
\Delta \epsilon, \text { vgr.), } 285 \text { (vgr.) }\end{array}$ & $\{$ Keph. 9: 285 (rezent) $\}$ \\
\hline 9,29 & $127(\div)$ & $\begin{array}{l}\text { 103, 108, 127, } 136 \text { (bei паl } \\
\text { Аe NTоч) }\end{array}$ & \\
\hline 9,30 & pbo & $\begin{array}{l}\text { 103, } 105 \text { (griechisch, vgr.), } \\
108,134,136 \text { (vgr.), } 174\end{array}$ & \\
\hline 9,31 & $\{\mathrm{pbo}\}$ & 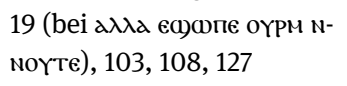 & \\
\hline $\begin{array}{l}9,32 \\
9,33\end{array}$ & & & \\
\hline 9,34 & & $\begin{array}{l}\text { 19, 103, } 105 \text { (griechisch, } \\
\text { vgr.), 108, 127, 134, 136, } 285\end{array}$ & \\
\hline 9,35 & $127(\dot{*})$ & $\begin{array}{l}\text { 19, 103, } 105 \text { (griechisch, } \\
\text { vgr.), 108, 126, 127, 134, } 142 \\
\text { (vgr.), } 285\end{array}$ & $\{$ Keph. 9: 285 (rezent) $\}$ \\
\hline 9,36 & & 126,136 & \\
\hline 9,37 & & 126,134 & \\
\hline 9,38 & $\{\mathrm{pbo}\}$ & $\begin{array}{l}105 \text { (griechisch, vgr.), 108, } \\
127,134\end{array}$ & 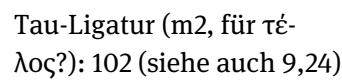 \\
\hline 9,39 & pbo, 102, $125($ (), $127(\div)$ & $\begin{array}{l}\text { 19, } 105 \text { (griechisch), 125, } \\
127,174,285\end{array}$ & \\
\hline
\end{tabular}




\begin{tabular}{|c|c|c|c|}
\hline & Paragraphenzeichen & Initialen & Nummerierungen / Varia \\
\hline 9,40 & 141 & $\begin{array}{l}19,108,103,126,127,174, \\
285\end{array}$ & \\
\hline 9,41 & & $126,174,285$ & \\
\hline 10,1 & 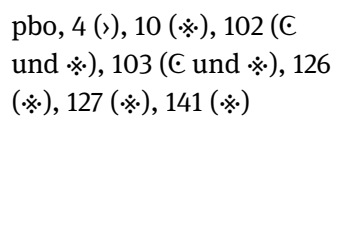 & $\begin{array}{l}\text { 4, } 9 \text { (vgr.), } 10 \text { (vgr.), } 19 \\
\text { (vgr.), 103, } 126 \text { (vgr.), 127, } \\
136 \text { (vgr.), } 141 \text { (vgr.), 174, } \\
285 \text { (vgr.) }\end{array}$ & $\begin{array}{l}\text { „Amen-Wort“ 15: 4; \{Keph. } \\
\text { 10: } 285 \text { (rezent)\}; Abschnitt } \\
\text { 23: } 10 \text { (m2), 109, } 126 \text { (nur } \\
\text { Titel), } 174 \text { (m2), 285; Glosse: } \\
\text { 103 (єхм оүархнєпскопос); } \\
\text { *: } 102 \text { (Ini.) }\end{array}$ \\
\hline 10,2 & & 285 & \\
\hline 10,3 & & $\begin{array}{l}103 \text { (bеi аүш саумоүте), } \\
136 \text { (vgr.) }\end{array}$ & \\
\hline 10,4 & & $19,127,136$ (vgr.) & \\
\hline 10,5 & & 136 & 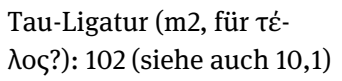 \\
\hline 10,6 & & $\begin{array}{l}9 \text { (vgr.), 103, 106, 127, 134, } \\
141,285\end{array}$ & \\
\hline 10,7 & $\begin{array}{l}\text { pbo, 4(>/৫), } 102(\div), 127 \\
(\stackrel{\bullet}{*}), 136(\dot{*})\end{array}$ & $\begin{array}{l}4 \text { (vgr.), } 9 \text { (vgr.), } 19 \text { (vgr.), } \\
\text { 103, 106, 126, 127, } 134 \\
\text { (vgr.), } 136 \text { (vgr.), } 285\end{array}$ & $\begin{array}{l}\text { „Amen-Wort“ 16: 4; 粶: } 102 \\
(\div)\end{array}$ \\
\hline 10,8 & & 103,126 & \\
\hline 10,9 & $\begin{array}{l}126 \text { (de facto є in epooy, } \\
\text { letztes Wort von } 10,8 \text { ) }\end{array}$ & 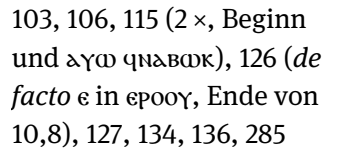 & \\
\hline 10,10 & $115(\dot{*} \cdot$ bei anok NTaleı) & $\begin{array}{l}\text { 103, } 115 \text { ( } 2 \times \text {, Beginn und a- } \\
\text { NoK NTalel, vgr.), 134, } 136\end{array}$ & \\
\hline 10,11 & $4(\odot), 10(\stackrel{*}{*})$ & $\begin{array}{l}4 \text { (vgr.), } 9 \text { (vgr.), } 10 \text { (vgr.), } \\
19 \text { (vgr.), 103, 106, } 115 \text { (2×, } \\
\text { Beginn und а)дчка тєч- } \\
\text { Үүхн), 127, 136, } 285 \text { (vgr.) }\end{array}$ & $\{$ Keph. 10: 285 (rezent) $\}$ \\
\hline 10,12 & & 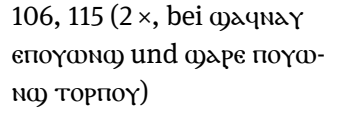 & \\
\hline \multicolumn{4}{|l|}{10,13} \\
\hline 10,14 & & $19,103,106,115,127,285$ & can. 90/III: 285 \\
\hline 10,15 & & 115,285 & can. 91/IV: 285 \\
\hline 10,16 & $4(>/ \odot)$ & $\begin{array}{l}\text { 4, } 103(2 \times \text {, Beginn und aүo } \\
\text { мєтммау }), 106,115,127,285\end{array}$ & can. 92/X: 285 \\
\hline 10,17 & & $103,115,285$ & $\{$ Keph. 10: 285 (rezent)\} \\
\hline 10,18 & & $\begin{array}{l}115 \text { (bei oүnt tezoYcla eka- } \\
\text { ac) }\end{array}$ & \\
\hline 10,19 & pbo, $10(\dot{*}), 101(\dot{*})$ & $\begin{array}{l}9 \text { (vgr.), 10, 19, 101, 103, } \\
115,127,285\end{array}$ & \\
\hline 10,20 & & 115,285 & \\
\hline
\end{tabular}




\begin{tabular}{|c|c|c|c|}
\hline & Paragraphenzeichen & Initialen & Nummerierungen / Varia \\
\hline 10,21 & & 19,127 & \\
\hline 10,22 & 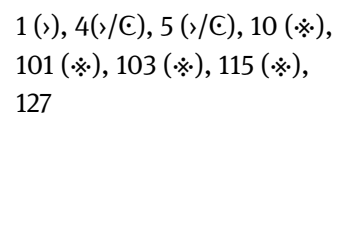 & $\begin{array}{l}4 \text { (vgr.), } 5 \text { (vgr.), } 9 \text { (vgr.), } 10 \\
\text { (vgr.), 101, 103, } 115 \text { (vgr.), } \\
\text { 127, } 142 \text { (vgr.), 197, } 285 \\
\text { (vgr.) }\end{array}$ & $\begin{array}{l}\text { \{Keph. 11: } 10 \text { (rezent)\}; Ab- } \\
\text { schnitt 24: } 10(\mathrm{~m} 2), 115 \\
\text { (m2), 285; Glosse: } 115 \text { (陑> } \\
\text { d > [zur Deutung siehe } \\
\text { 4.5.]); Rubrizierung: } 197 \\
\text { (Vers in Rot) }\end{array}$ \\
\hline 10,23 & $\{\mathrm{pbo}\}$ & & \\
\hline 10,24 & & $\begin{array}{l}103 \text { (bei ec) } x \in \text { nток), 115, } \\
285\end{array}$ & \\
\hline 10,25 & pbo, 647 & $19,103,115,142$ & \\
\hline 10,26 & & 115,142 (vgr.) & \\
\hline 10,27 & & 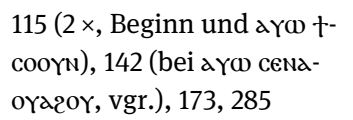 & $\{$ Keph. 10: 285 (rezent) $\}$ \\
\hline 10,28 & & 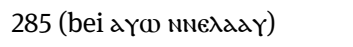 & \\
\hline 10,29 & & 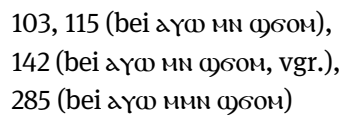 & \\
\hline 10,30 & & 115,142 (vgr.) & \\
\hline 10,31 & $127(\div)$ & $19,115,127,173$ & \\
\hline 10,32 & pbo & $103,115,127,285$ & \\
\hline 10,33 & pbo & 19, 103, 115, 142 (vgr.), 285 & \\
\hline 10,34 & pbo, $127(\div)$ & $\begin{array}{l}\text { 19, 103, 115, 127, } 142 \text { (vgr.), } \\
285\end{array}$ & $\{$ Keph. 10: 285 (rezent) $\}$ \\
\hline 10,35 & & $\begin{array}{l}103(2 \times \text {, Beginn und aүळ M- } \\
\text { мN6ом), } 285\end{array}$ & \\
\hline 10,36 & & & \\
\hline 10,37 & & 103,142 (vgr.) & \\
\hline 10,38 & & $\begin{array}{l}\text { 103, } 115 \text { ( } 2 \times \text {, Beginn und } \\
\text { xєкас єтєтмєєімє), } 142 \text { (bei } \\
\text { aүФ амок, vgr.) }\end{array}$ & \\
\hline 10,39 & & $19,103,115,127,285$ & \\
\hline 10,40 & & 142 (vgr.), 197 & \\
\hline 10,41 & & 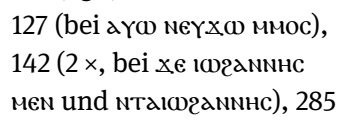 & can. 94/X: 285 \\
\hline 10,42 & & 142 & \\
\hline 11,1 & $\begin{array}{l}\text { pbo, } 1(>), 4(>/ \odot), 5(>/ \odot), 10 \\
(\dot{*}), 103(\dot{*}), 127(\dot{*})\end{array}$ & $\begin{array}{l}\text { 4, 5, } 9 \text { (vgr.), } 10 \text { (vgr.), 19, } \\
103 \text { ( } 2 \times \text {, Beginn und 2N BH- } \\
\text { edNid), 115, 127, } 142 \text { (vgr.), } \\
\text { 197, } 285 \text { (vgr.) }\end{array}$ & $\begin{array}{l}\text { Keph. 10: 9; Keph. 11: }\{10 \\
\text { (rezent)\}, 142, *197 (Titel } \\
\text { unter der Kol.), } 285 \text { (* und } \\
\text { rezent); Abschnitt 25: } 115 \\
\text { (m2), 285; Abschnitt 26: } 10 \\
\text { (m2); Rubrizierung: } 197 \\
\text { (Beginn von 11,1 in Rot) }\end{array}$ \\
\hline
\end{tabular}




\begin{tabular}{|c|c|c|c|}
\hline & Paragraphenzeichen & Initialen & Nummerierungen / Varia \\
\hline \multicolumn{4}{|l|}{11,2} \\
\hline 11,3 & & $19,103,115$ & \\
\hline 11,4 & pbo & 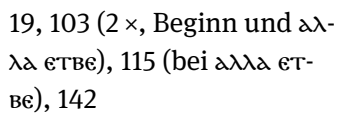 & $\begin{array}{l}81 \text { (Punkt am Ende der Zeile } \\
\text { mit diesem Vers) }\end{array}$ \\
\hline 11,5 & & $103,115,142$ & \\
\hline 11,6 & pbo & $\begin{array}{l}103(2 \times \text {, Beginn und тоте } \\
\text { меN), } 115(2 \times \text {, Beginn und } \\
\text { тоте меN })\end{array}$ & \\
\hline 11,7 & & 19,115 & \\
\hline 11,8 & & 285 & $\{$ Keph. 11: 285 (rezent) $\}$ \\
\hline 11,9 & pbo & $\begin{array}{l}\text { 19, 127, } 285(2 \times, \text { Beginn } \\
\text { und ерсаноүа мооб)є) }\end{array}$ & \\
\hline 11,10 & & $115,127,285$ & \\
\hline 11,11 & $\begin{array}{l}\text { pbo, (127, } \dot{*} \text {, Position unsi- } \\
\text { cher, auch 11,10 möglich) }\end{array}$ & $103,108,127,285$ & \\
\hline 11,12 & & $19,103,285$ & \\
\hline 11,13 & & 103 & $\begin{array}{l}81 \text { (Punkt am Zeilenbeginn } \\
\text { mit dem Vers, Sinn unklar) }\end{array}$ \\
\hline 11,14 & pbo & 103,285 & \\
\hline \multicolumn{4}{|l|}{11,15} \\
\hline 11,16 & 4 & $4,19,103,108,285$ & $\{$ Keph.: 285 (rezent) $\}$ \\
\hline 11,17 & $\{\mathrm{pbo}\}$ & 19 & $\begin{array}{l}\text { Glosse: pbo (ппа̣yl r, Ober- } \\
\text { rand, hierher?, Sinn } \\
\text { unklar) }\end{array}$ \\
\hline 11,18 & $4(>/ \odot)$ & 4, 19, 103, 127, 285 (vgr.) & \\
\hline \multicolumn{4}{|l|}{11,19} \\
\hline 11,20 & & 19, 103 (bei мapla $\triangle$ є), 285 & \\
\hline 11,21 & 4 & $4,127,285$ & \\
\hline \multicolumn{4}{|l|}{11,22} \\
\hline 11,23 & & 19,127 & \\
\hline 11,24 & & 103 & \\
\hline 11,25 & & $\begin{array}{l}\text { 103, } 127 \text { (bei пєтпстеүє), } \\
285(2 \times \text {, Beginn und } \\
\text { петпістєүє) }\end{array}$ & \{Keph. 11: 285 (rezent)\} \\
\hline 11,26 & $\{\mathrm{pbo}\}$ & & \\
\hline \multicolumn{4}{|l|}{11,27} \\
\hline 11,28 & $\dagger 109$ & 19, 103, 199 & \\
\hline 11,29 & & 103, 127, 199, 285 & \\
\hline 11,30 & & 199 & \\
\hline 11,31 & & $\begin{array}{l}\text { 19, 127, } 199 \text { (Beginn und N- } \\
\text { тероүмаү емдрід) }\end{array}$ & \\
\hline 11,32 & & $19,103,199,285$ & \\
\hline 11,33 & & 19, 103, 127, 155 (vgr.), 199 & \\
\hline 11,34 & $199(\div$ bei пехаY Nay $х є)$ & $\begin{array}{l}108 \text { (bei пехау мач } х є) \text { ), 142, } \\
199\end{array}$ & \\
\hline
\end{tabular}




\begin{tabular}{|c|c|c|c|}
\hline & Paragraphenzeichen & Initialen & Nummerierungen / Varia \\
\hline \multicolumn{4}{|l|}{11,35} \\
\hline \multicolumn{4}{|l|}{11,36} \\
\hline 11,37 & $\begin{array}{l}199(\div \text { bei емм } 6 \text { [ом] ммоч } \\
\text { оN) }\end{array}$ & 199 & \\
\hline 11,38 & $4(>)$ & $\begin{array}{l}\text { 4, 19, 103, } 115 \text { (vgr.), 127, } \\
142 \text { (vgr.), } 155 \text { (vgr.) }\end{array}$ & \\
\hline 11,39 & & $\begin{array}{l}115(2 \times \text {, Beginn und пехє } \\
\text { марөа), } 127\end{array}$ & \\
\hline 11,40 & $199(>)$ & $103,115,142,199$ & \\
\hline 11,41 & & 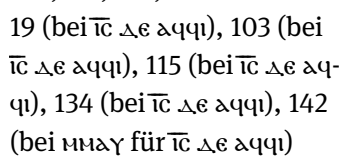 & \\
\hline 11,42 & & 115,199 & Zitat $\rightarrow »: 116$ \\
\hline 11,43 & 116 & $19,103,115,116,127$ & \\
\hline 11,44 & $\begin{array}{l}\text { pbo, } 199 \text { (am Beginn } \odot \text { und } \\
\div \text { bei nтетNкдач) }\end{array}$ & $\begin{array}{l}\text { 19, } 115 \text { (bei пєхє } \overline{\mathrm{IC}}), 142 \\
\text { (vgr.), } 199(2 \times, \text { Beginn und } \\
\text { пєхє } \overline{\mathrm{IC}})\end{array}$ & \\
\hline 11,45 & $139(\dot{*})$ & $\begin{array}{l}\text { 103, 115, 127, 134, 139, 142, } \\
199\end{array}$ & \$: 127 (ткүріакн NCION) \\
\hline 11,46 & $116(\dot{*})$ & $\begin{array}{l}\text { 105, 115, } 116 \text { (vgr.), 127, 134, } \\
139 \text { (vgr.) }\end{array}$ & \\
\hline 11,47 & $131(\div$ mit $\dot{x})$ & $\begin{array}{l}19 \text { (vgr.), 103, 115, 131, 134, } \\
139\end{array}$ & \\
\hline \multicolumn{4}{|l|}{11,48} \\
\hline 11,49 & $\begin{array}{l}115(\stackrel{*}{*}), 116(\div \text { und } \odot), 131 \\
\text { (obelosartige } \dot{*} \text { ) }\end{array}$ & $\begin{array}{l}\text { 9, } 19 \text { (vgr.), 103, } 115 \text { (vgr.), } \\
116,127,131,139\end{array}$ & \\
\hline \multicolumn{4}{|l|}{11,50} \\
\hline 11,51 & & $\begin{array}{l}103(2 \times, \text { Beginn und дчпро- } \\
\text { фнтеүє), 127, } 134\end{array}$ & \\
\hline 11,52 & & $\begin{array}{l}115(2 \times \text {, Beginn und } \lambda \lambda \lambda d \\
\text { xeKac) }\end{array}$ & \\
\hline 11,53 & $131(\div)$ & 115 (vgr.), 131, 134 & can. 95/IV: 131 \\
\hline 11,54 & 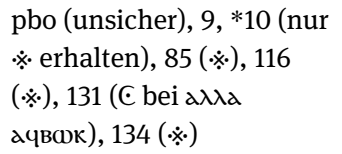 & $\begin{array}{l}\text { 9, *10 (vgr., nur } * \text { erhal- } \\
\text { ten), } 19 \text { (vgr.), } 85 \text { (vgr.), } 115 \\
\text { (vgr.), } 116 \text { (vgr.), 127, } 134 \\
\text { (vgr.), } 136\end{array}$ & 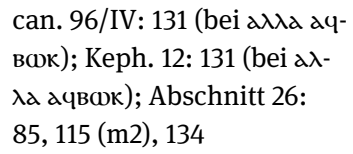 \\
\hline 11,55 & $131(\odot)$ & 9 (vgr.), 19, 108, 115, 131, 134 & can. 97/X: 131 \\
\hline 11,56 & & 115,139 & \\
\hline 11,57 & $154(\dot{*})$ & $112,115,134,139,154$ & \\
\hline 12,1 & $\begin{array}{l}4(>/ \odot), 10(\dot{*}), 112(\cdot \dot{*}), 116 \\
(\dot{*}), 127(\cdot \dot{*}), 131(\odot), 134 \\
(\dot{*}), 139(\dot{*})\end{array}$ & $\begin{array}{l}4 \text { (vgr.), } 9 \text { (vgr.), } 10 \text { (vgr.), } \\
19 \text { (vgr.), 108, } 116 \text { (vgr.), } \\
\text { 127, 131, } 134 \text { (vgr.), } 139\end{array}$ & $\begin{array}{l}\text { Keph. 11: 9; Abschnitt 27: } 10 \\
\text { (m2), } 108 \text { (wohl m2), 116, } \\
\text { 134, } 139(\mathrm{~m} 2) ; \text { Abschnitt 27: } \\
\text { *12 (m2, } \overline{\text { KH}) ; ~ G l o s s e: ~} 154 \text { (т- } \\
\text { күріגкн } \triangle \text { ) }\end{array}$ \\
\hline
\end{tabular}




\begin{tabular}{|c|c|c|c|}
\hline & Paragraphenzeichen & Initialen & Nummerierungen / Varia \\
\hline 12,2 & $\begin{array}{l}131(\div), 154(\div \text { bei } \lambda a z a p o c \\
\text { Ge Neoүa) }\end{array}$ & 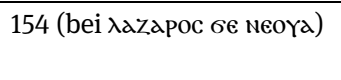 & can. 98/IV: 131 \\
\hline 12,3 & $4,131(\div)$ & 4,136 & \\
\hline 12,4 & $131(\odot)$ & 9 (vgr.), 19 & $\begin{array}{l}\text { Keph. 12: 9; Keph. 13: }\{10 \\
\text { (rezent)\}, } 131\end{array}$ \\
\hline \multicolumn{4}{|l|}{12,5} \\
\hline 12,6 & & 127,134 & \\
\hline 12,7 & $131(\div)$ & $19,131,134$ & \\
\hline \multicolumn{4}{|l|}{12,8} \\
\hline 12,9 & $\begin{array}{l}131 \text { (obelosartige } \dot{*}), 154 \\
(\dot{*})\end{array}$ & $19,127,134,154$ & can. 99/X: 131 \\
\hline 12,10 & $154(\dot{*})$ & 134,154 & \\
\hline \multicolumn{4}{|l|}{12,11} \\
\hline 12,12 & 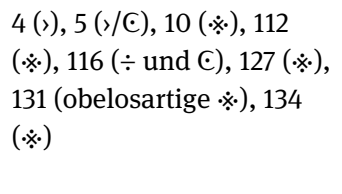 & $\begin{array}{l}\text { 4, 5, } 9 \text { (vgr.), 10, 19, } 112 \\
\text { (vgr.), 116, 127, 131, } 134 \\
\text { (vgr.), 136 (vgr.), } 142 \text { (vgr.), } \\
285 \text { (vgr.) }\end{array}$ & $\begin{array}{l}\text { can. 100/I: 131, 285; }\{\text { Keph. } \\
\text { 12: } 285 \text { (rezent)\}; Keph. 13: } \\
\text { 9; Keph. 14: }\{10 \text { (rezent)\}, } \\
\text { 131, } 285 \text { [*]; Abschnitt 28: } \\
\text { 285 }\end{array}$ \\
\hline 12,13 & $112\left(\mathbb{\Phi}\right.$ und $\left.^{\circ \circ}\right)$ & 112 (bei аүє1 єво入 єтФммт) & \\
\hline 12,14 & 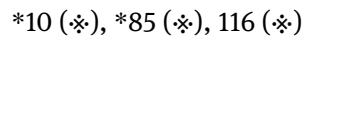 & $\begin{array}{l}* 10 \text { (vgr.), 19, *85 (vgr.), } \\
112,116 \text { (vgr.), 127, 142, } 285\end{array}$ & $\begin{array}{l}\text { Zitat } \rightarrow »: 1 \text {, 4; Abschnitt } \\
28: * 10(\mathrm{~m} 2 \text {, fast unleser- } \\
\text { lich), } 116\end{array}$ \\
\hline 12,15 & & & Zitat $\rightarrow »: 1,4$ \\
\hline 12,16 & pbo, 131 & 127, 285 & $\begin{array}{l}\text { can. 102/X: *131 (Spuren), } \\
285\end{array}$ \\
\hline 12,17 & & 136 (vgr.), 142 (vgr.), 285 & \\
\hline 12,18 & $131(\div)$ & $131,142,285$ & \{Keph. 12: 285 (rezent)\} \\
\hline 12,19 & & 127 & \\
\hline 12,20 & $\begin{array}{l}\text { pbo, } 4(>), 10(\dot{*}), 116(\dot{*}), \\
127 \text { ( } \dot{*}, \text { erste Verszeile vgr.) }\end{array}$ & $\begin{array}{l}\text { 4, } 9 \text { (vgr.), } 10 \text { (vgr.), 19, } 116 \\
\text { (vgr.), 127, } 285 \text { (vgr.) }\end{array}$ & $\begin{array}{l}\text { Keph. 14: 9; Keph. 15: }\{10 \\
\text { (rezent, fast unleserlich)\}, } \\
131 \text { (Titel), } 285[*] ; \text { Abschnitt } \\
29: * 10(\mathrm{~m} 2 \text {, fast } \\
\text { unleserlich), } 116(\mathrm{~m} 2), 285\end{array}$ \\
\hline
\end{tabular}

12,21

12,22

106, 136, 285

12,23 pbo, $\{* 131$ (verloren) $\} \quad 19,131,139,285$

$12,24 \quad 4(>), * 106(\cdot \dot{*}), 116(\cdot \dot{*})$

4, *106, 116 (vgr.), 127, 136

can. 103/IV: *131, 285

(vgr.), 139 (vgr.), 173, 285

„Amen-Wort“ 17: 4; can.

104/X: 285

12,25

285

can. 105/III: 285

12,26 $106(\stackrel{\times}{*}), 116$ (※. unvollen- 19 (bei еро)амоүa), 106, 127

det, sicher von $\mathrm{m} 2), 141(>) \quad(2 \times$, Beginn und ep( $)$ anoya), $136(2 \times$, Beginn und ер()амоүа), 141 


\begin{tabular}{|c|c|c|c|}
\hline & Paragraphenzeichen & Initialen & Nummerierungen / Varia \\
\hline 12,27 & $\begin{array}{l}106(\dot{\times}), 116(\div \text { und } \odot), 131 \\
(2 \times, \text { Beginn, } \div \text {, und } \\
\text { паеєот матоү } \times \text { ol, } \div), 141 \\
(>)\end{array}$ & 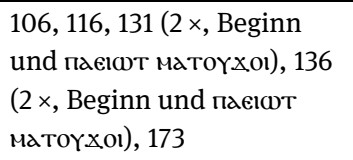 & $\begin{array}{l}\text { can. 107/IV: 131; can. 108/X: } \\
131 \text { (bei пдєІळт матоүхоı) }\end{array}$ \\
\hline 12,28 & $131(\div)$ & 131 & \\
\hline 12,29 & $\begin{array}{l}106(>\text { bei } 2 \text { enkooye), } 116(\div \\
\text { und } \odot), * 131 \text { (Spuren von } \\
\div), 141(\dot{*})\end{array}$ & $\begin{array}{l}\text { 10, 19, } 106 \text { (bei гєnкооүє, } \\
\text { *auch Beginn?), } 116 \text { (vgr.), } \\
\text { 131, } 141 \text { (vgr.), } 173\end{array}$ & \\
\hline 12,30 & pbo & 127,173 & \\
\hline 12,31 & $\begin{array}{l}\text { pbo (bei †NoY пархФN), } 141 \\
(2 \times, \text {, Beginn und темоY ח- } \\
\text { архФN) }\end{array}$ & 141 & \\
\hline 12,32 & & & \\
\hline 12,33 & $116(\div$ und $\odot), 141(>)$ & 116,127 & \\
\hline 12,34 & pbo, $141(>)$ & $\begin{array}{l}\text { 19, } 127 \text { ( } 2 \times \text {, Beginn und мім } \\
\text { пе пюбнре), } 141 \text { (vgr.) }\end{array}$ & \\
\hline 12,35 & $4(>), 116(\div$ und $\odot)$ & $\begin{array}{l}\text { 4, 19, 106, 116, } 127 \text { (bei го- } \\
\text { con оүнтетм), } 173\end{array}$ & \\
\hline 12,36 & $\begin{array}{l}10(\dot{*}), 116(\dot{*} \text { bei nal } \\
\text { духооҮ), } 127(\div \text { bei хекас } \\
\text { ететмє)(пп) }\end{array}$ & 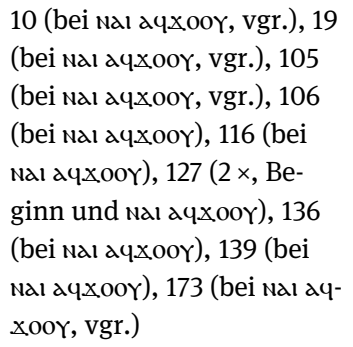 & 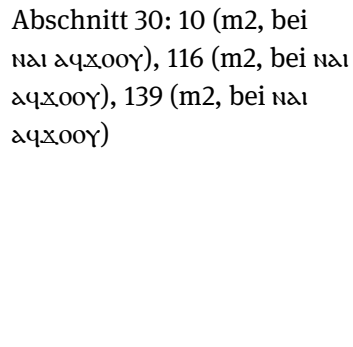 \\
\hline 12,37 & & 139,142 & \\
\hline 12,38 & & 103 & $\begin{array}{l}\text { Zitat } \rightarrow »: 1,4,5,10,19 \\
\text { (Punkte), } 116 \text { (Punkte) }\end{array}$ \\
\hline 12,39 & & 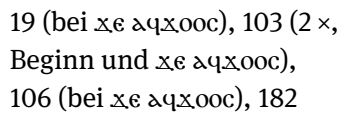 & Zitat $\rightarrow »:$ 4, 5, 116 (Punkte) \\
\hline 12,40 & & 103 (bei xeкac nNeүNaY) & $\begin{array}{l}\text { Zitat } \rightarrow »: 1,4,5,10,101, \\
116 \text { (Punkte) }\end{array}$ \\
\hline 12,41 & pbo, 4(>), $101(\stackrel{*}{*}), 105,116$ & $\begin{array}{l}4 \text { (vgr.), } 19 \text { (vgr.), 101, 103, } \\
105,106,116,127,182\end{array}$ & \\
\hline 12,42 & $116(\div$ und $\odot)$ & 116,127 & \\
\hline 12,43 & & 127 & \\
\hline 12,44 & $\begin{array}{l}4(>), 103(\cdot \dot{*}), 105,116(\dot{*}), \\
154(\dot{*})\end{array}$ & $\begin{array}{l}4 \text { (vgr.), } 9 \text { (vgr.), 19, 103, } \\
105 \text { (vgr.), } 116 \text { (vgr.), 127, } \\
\text { 154, } 182 \text { (vgr.) }\end{array}$ & 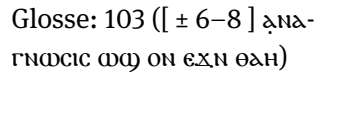 \\
\hline \multicolumn{4}{|l|}{12,45} \\
\hline $\begin{array}{l}12,46 \\
12,47\end{array}$ & 154 & $\begin{array}{l}19,106,127,154,182 \\
\{10 \text { (Spuren von Ini., exakte } \\
\text { Phrase unsicher) }\}\end{array}$ & \\
\hline
\end{tabular}




\begin{tabular}{|c|c|c|c|}
\hline & Paragraphenzeichen & Initialen & Nummerierungen / Varia \\
\hline 12,48 & & 103, 127 (bei па)дхe) & \\
\hline 12,49 & $141(>)$ & 103,141 & \\
\hline 12,50 & $141(>)$ & 103, 127 (bei ne†хм 6є), 141 & \\
\hline 13,1 & 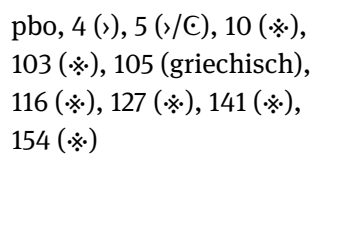 & $\begin{array}{l}\text { 1, } 4 \text { (vgr.), } 9 \text { (vgr.), } 10 \text { (vgr), } \\
19 \text { (vgr.), } 103 \text { (vgr.), } 105 \\
\text { (griechisch, vgr.), 106, } 116 \\
\text { (vgr.), } 126 \text { (vgr.), 127, } 132 \\
\text { (vgr.), } 141 \text { (vgr.), } 154 \text { (vgr.), } \\
182 \text { (vgr.) }\end{array}$ & $\begin{array}{l}\text { Keph. 15: 9; Abschnitt 31: } 10 \\
\text { (m2), } 116 \text { (m2), } 126 \text { (nur } \\
\text { Titel), 132, } 182\end{array}$ \\
\hline 13,2 & $141(\dot{*}), 154(\dot{*})$ & $\begin{array}{l}19 \text { (vgr.), } 105 \text { (griechisch), } \\
\text { 106, } 141 \text { (vgr.), } 154\end{array}$ & \\
\hline 13,3 & $141(>), * 243$ & $\begin{array}{l}\text { 103, } 105 \text { (griechisch), 108, } \\
141\end{array}$ & \\
\hline 13,4 & $141(>)$ & 103, 106, 108, 132 (vgr.), 141 & Keph. 16: 132 \\
\hline 13,5 & $141(>)$ & 141 & \\
\hline 13,6 & $141(>)$ & $19,103,106,139,141$ & \\
\hline 13,7 & pbo & $19,106,108,142$ (vgr.) & \\
\hline 13,8 & 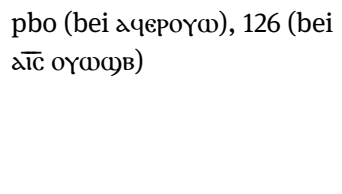 & 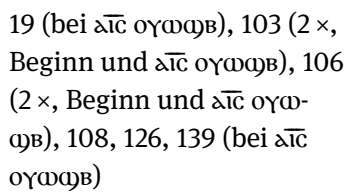 & \\
\hline \multicolumn{4}{|c|}{ 1. } \\
\hline 13,10 & & 19,126 & \\
\hline 13,11 & & $\begin{array}{l}142 \text { (bei етвє паl аухоос, } \\
\text { vgr.) }\end{array}$ & \\
\hline 13,12 & 4 & $\begin{array}{l}\text { 4, 19, } 103 \text { (vgr.), } 126 \text { (vgr.), } \\
139 \text { (vgr.), } 142 \text { (bei пєхגч } \\
\text { NaY Хe, vgr.) }\end{array}$ & \\
\hline \multicolumn{4}{|c|}{. } \\
\hline 13,14 & $\begin{array}{l}* 266(\odot, \text { fragm., wohl zu } \\
\text { diesem Vers) }\end{array}$ & $139,\{266\}$ & \\
\hline 13,15 & & 103 & \\
\hline \multicolumn{3}{|l|}{13,17} & „Amen-Wort“ 18: 4 \\
\hline 13,18 & & 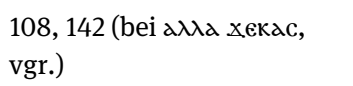 & Zitat $\rightarrow »: 1,4$ \\
\hline 13,19 & & 103 & \\
\hline 13,20 & pbo, $4(>), 103,154(\stackrel{*}{*})$ & $\begin{array}{l}\text { 4, } 9 \text { (vgr.), } 19 \text { (vgr.), } 103 \\
\text { (vgr.), 108, } 139 \text { (vgr.), } 154\end{array}$ & $\begin{array}{l}\text { „Amen-Wort“ 19: 4; Ab- } \\
\text { schnitt 32: 108, } 139 \text { (m2) }\end{array}$ \\
\hline 13,21 & $\begin{array}{l}\text { pbo (bеi хе гамнм гамнн), } \\
5,10(\dot{*})\end{array}$ & $\begin{array}{l}\text { 9, } 10 \text { (vgr.), 108, 124, } 142 \\
\text { (vgr.), } 154 \text { (bei хє гамнм } \\
\text { 2амнм) }\end{array}$ & $\begin{array}{l}\text { „Amen-Wort“ 20: 4; Ab- } \\
\text { schnitt 32: } 10\end{array}$ \\
\hline 13,22 & & 108,124 & \\
\hline 13,23 & & $19,124,139,154$ & 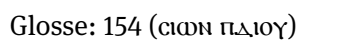 \\
\hline
\end{tabular}




\begin{tabular}{|c|c|c|c|}
\hline & Paragraphenzeichen & Initialen & Nummerierungen / Varia \\
\hline 13,24 & & 19,124 & \\
\hline 13,25 & 124 & 108 & \\
\hline 13,26 & pbo, $154(\stackrel{*}{*})$ & 108, 124, 139, 154 (vgr.) & \\
\hline 13,27 & & 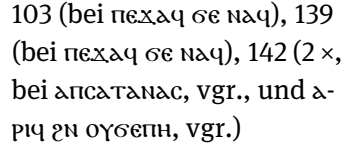 & \\
\hline 13,28 & & & \\
\hline 13,29 & & $\begin{array}{l}\text { 103, 124, } 142 \text { (bеі н хекас } \\
\text { eet十, vgr.), } 154\end{array}$ & \\
\hline 13,30 & & $19,124,139,154$ & \\
\hline 13,31 & 124 & 103,154 & \\
\hline 13,32 & & $\begin{array}{l}142 \text { (bei aүळ ппоҮте nat } \\
\text { eоoү, vgr.) }\end{array}$ & \\
\hline 13,33 & $4(>/ \odot), 5(>/ \odot)$ & $\begin{array}{l}4 \text { (vgr.), 5, } 9 \text { (vgr.), 19, 108, } \\
124 \text { (vgr.), } 139 \text { (vgr.), } 142 \text { ( } \\
\times \text {, Beginn, vgr., und }+\times 1 \text { м- } \\
\text { Moc мнTN, vgr.) }\end{array}$ & \\
\hline 13,34 & & 139 & \\
\hline 13,35 & $57(>)$ & 124 & \\
\hline 13,36 & pbo (bei ачєроүळ) & $\begin{array}{l}\text { 19, 108, 124, } 139 \text { (bei } \\
\text { ачоүсовв мач) }\end{array}$ & \\
\hline 13,37 & & 19,124 & \\
\hline 13,38 & $\begin{array}{l}\text { pbo, } 4 \text { (> bei гамнм гамнм), } \\
12\end{array}$ & $\begin{array}{l}4 \text { (bei гамнм гамнм), 5, 19, } \\
\text { 108, 124, 139, } 142 \text { (vgr.) }\end{array}$ & „Amen-Wort“ 21: 4 \\
\hline 14,1 & & $\begin{array}{l}9 \text { (vgr.), } 10 \text { (vgr.), } 19 \text { (vgr.), } \\
\text { 108, } 124 \text { (vgr.), 139, } 142 \\
\text { (vgr.) }\end{array}$ & $\begin{array}{l}\text { Abschnitt 33: } 10 \text { (m2), 108, } \\
124 \text { (m2), } 139 \text { (m2); Glosse: } \\
124 \text { (m2, Unterrand, unle- } \\
\text { serlich, Titel?) }\end{array}$ \\
\hline \multicolumn{4}{|l|}{14,2} \\
\hline \multicolumn{4}{|l|}{14,3} \\
\hline \multicolumn{4}{|l|}{14,4} \\
\hline 14,5 & & $9,19,139$ & \\
\hline 14,6 & & 19, 142 (vgr.) & \\
\hline 14,7 & & 139 & \\
\hline 14,8 & & $9,19,108,139$ (vgr.) & \\
\hline 14,9 & $\{\mathrm{pbo}\}$ & 108 & \\
\hline 14,10 & & 19 (bei м⿻ахає анок $\epsilon+x \omega)$ & \\
\hline 14,11 & & 19 & \\
\hline 14,12 & pbo, $4(>), 116(\dot{*})$ & $\begin{array}{l}\text { 4, } 9 \text { (vgr.), *19, 108, } 116 \\
\text { (vgr.), } 142 \text { (bei аүळ чмар) }\end{array}$ & „Amen-Wort“ 22: 4 \\
\hline 14,13 & & 108, 142 (bei хєкаc) & \\
\hline \multicolumn{4}{|l|}{14,14} \\
\hline \multicolumn{4}{|l|}{14,15} \\
\hline 14,16 & & 142 (vgr.) & \\
\hline
\end{tabular}




\begin{tabular}{|c|c|c|c|}
\hline & Paragraphenzeichen & Initialen & Nummerierungen / Varia \\
\hline 14,17 & pbo & 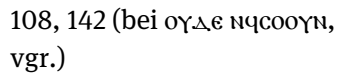 & \\
\hline 14,18 & $4(>), 116(\div$ und $\odot), 141(\stackrel{*}{*})$ & $\begin{array}{l}\text { 4, } 9 \text { (vgr.), } 19 \text { (vgr.), 108, } \\
\text { 116, } 139 \text { (vgr.), } 141 \text { (vgr.) }\end{array}$ & \\
\hline \multicolumn{4}{|l|}{14,19} \\
\hline 14,20 & pbo (bei тетенnаємi) & & \\
\hline 14,21 & $4(>)$ & $\begin{array}{l}\text { 4, } 19 \text { (vgr.), } 124 \text { (vgr.), } 139 \\
\text { (vgr.) }\end{array}$ & $\begin{array}{l}4 \text { („Liebesgebot“, abgesetzt } \\
\text { durch ») }\end{array}$ \\
\hline 14,22 & $116(\div$ und $\odot)$ & $19,116,124,139,286$ & \\
\hline 14,23 & pbo & $19,108,124,139$ & \\
\hline 14,24 & & $19,108,139$ & $\begin{array}{l}10(\cdot \dot{*} \text { von m2, unfertig, } \\
\text { wohl ohne Bedeutung })\end{array}$ \\
\hline 14,25 & & 139 & \\
\hline 14,26 & $10(\dot{*}), 116(\dot{*}), 286(\dot{x})$ & $\begin{array}{l}9 \text { (vgr.), } 10 \text { (vgr.), } 19 \text { (vgr.), } \\
\text { 108, } 116 \text { (erste Verszeile } \\
\text { vgr.), } 124 \text { (vgr.), 139, } 286\end{array}$ & $\begin{array}{l}\text { Keph. 16: 9; Abschnitt 34: } \\
10 \text { (m2), 108, } 116(\mathrm{~m} 2), 124 \text {, } \\
139(\mathrm{~m} 2), 286\end{array}$ \\
\hline 14,27 & $4(>)$ & $\begin{array}{l}\text { 4, } 9 \text { (bеі мпртрєпєтмгнт), } \\
\text { 10, } 19 \text { (bеі мпртрєпєтмгнт, } \\
\text { vgr.), 108, } 124 \text { ( } 2 \times \text {, Beginn, } \\
\text { vgr., und мпртрєпєтм2нт, } \\
\text { vgr.), } 139 \text { (vgr.) }\end{array}$ & \\
\hline \multicolumn{4}{|l|}{14,28} \\
\hline \multicolumn{4}{|l|}{14,29} \\
\hline 14,30 & pbo (bеi чмноү гар) & $19,103,108,124$ & \\
\hline 14,31 & 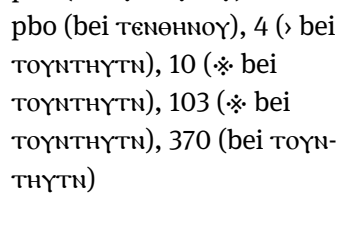 & 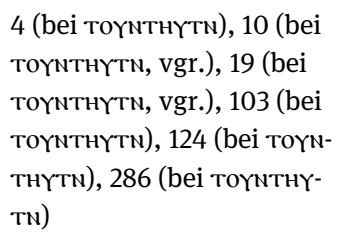 & \\
\hline 15,1 & $5(>/ \odot)$ & 9 (vgr.), 139 (vgr.) & \\
\hline 15,2 & & 103 (bei $а \gamma \omega$ ()ג2 мIM) & \\
\hline 15,3 & & 139 & \\
\hline 15,4 & & 123,124 & \\
\hline 15,5 & & 19, 103 (vgr.) & \\
\hline 15,6 & & 108,124 & \\
\hline 15,7 & $4(>), 10(\cdot \dot{x} \cdot \operatorname{von} \mathrm{m} 2)$ & $\begin{array}{l}\text { 4, } 103 \text { (vgr.), 108, } 124 \text { (vgr.), } \\
286\end{array}$ & \\
\hline 15,8 & & $19,103,108,286$ & 粶(?): 124 (Punktkreuz, m2) \\
\hline \multicolumn{4}{|l|}{15,9} \\
\hline 15,10 & & 103 (vgr.), 108 & \\
\hline 15,11 & pbo, $4(>/ €)$ & $\begin{array}{l}4 \text { (vgr.), } 9 \text { (vgr.), 19, 123, } 124 \\
\text { (vgr.) }\end{array}$ & \\
\hline 15,12 & & 19, 103 (vgr.), 124 & \\
\hline 15,13 & & 103 (vgr.), 108 & \\
\hline
\end{tabular}




\begin{tabular}{|c|c|c|c|}
\hline & Paragraphenzeichen & Initialen & Nummerierungen / Varia \\
\hline 15,14 & 103 & 103, 123, 124 (vgr.) & \\
\hline 15,15 & 162 & 19 (vgr.), 286 & 娄(?): 124 Punktkreuz, m2) \\
\hline 15,16 & & $\begin{array}{l}103 \text { ( } 2 \times \text {, Beginn, vgr., und } \\
\text { дүळ мте пєтмкарпос, vgr.), } \\
123 \text { (bei дүळ дікג тнүтN) }\end{array}$ & \\
\hline 15,17 & $\begin{array}{l}\text { pbo, } 109(\div), 207 \text { (bei } \\
\text { єт[єтмемере) }\end{array}$ & 19,103 (vgr.) & \\
\hline 15,18 & & 103 (vgr.), 108, 124, 286 & \\
\hline 15,19 & pbo (bei отı мєN) & 103 & \\
\hline 15,20 & & 19 (vgr.), 103 (vgr.), 123, 124 & \\
\hline \multicolumn{4}{|l|}{15,21} \\
\hline 15,22 & & 19, 103 (vgr.), 124 & \\
\hline 15,23 & & 19, 103 (vgr.), 139 & \\
\hline 15,24 & & 123 (bеi аүळ аүместюı) & \\
\hline 15,25 & & 124 & Zitat $\rightarrow »: 1,4$ \\
\hline 15,26 & $\begin{array}{l}\text { pbo, *10 }(\cdot \dot{*}), 103(\stackrel{*}{*}), 123 \text {, } \\
162\end{array}$ & $\begin{array}{l}9 \text { (vgr.), *10, } 19 \text { (vgr.), 103, } \\
123,124 \text { (vgr.), *139, } 286 \\
\text { (vgr.) }\end{array}$ & 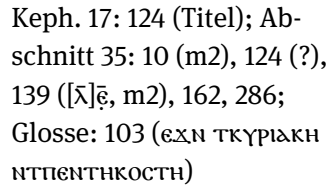 \\
\hline 15,27 & & 103 & \\
\hline 16,1 & & 9 (vgr.), 103 (vgr.), 124, 139 & \\
\hline \multicolumn{4}{|l|}{16,2} \\
\hline 16,3 & pbo & & \\
\hline 16,4 & $109,141(>)$ & $\begin{array}{l}9 \text { (vgr.), 19, } 103 \text { (vgr.), } 124 \text { (2 } \\
\times \text {, Beginn und Nal } \triangle є \text { мпєا- } \\
\text { xoor), } 139,141\end{array}$ & \\
\hline 16,5 & $141(>)$ & 103 (vgr.), 141 & \\
\hline 16,6 & & 124 & \\
\hline 16,7 & 141 (> bei ср Noчpe NHTN) & 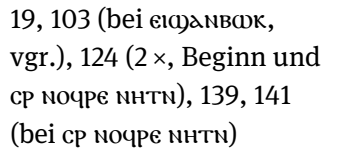 & \\
\hline 16,8 & & & Vers $\rightarrow »: 141$ \\
\hline 16,9 & & 106,124 & Vers $\rightarrow »: 141$ \\
\hline \multicolumn{4}{|l|}{16,10} \\
\hline 16,11 & & 124 & \\
\hline 16,12 & & 19,139 & \\
\hline 16,13 & $141(\dot{*})$ & $\begin{array}{l}19 \text { (vgr.), } 106 \text { ( } 2 \times \text {, Beginn } \\
\text { und neyna)d.xe), } 124 \text { (vgr.), } \\
139 \text { (vgr.), } 141 \text { (vgr.) }\end{array}$ & \\
\hline \multicolumn{4}{|l|}{16,14} \\
\hline 16,15 & $141(>)$ & 108, 139 (vgr.), 141 & \\
\hline 16,16 & & 108,139 & \\
\hline 16,17 & $141(>)$ & 19,141 & \\
\hline
\end{tabular}




\begin{tabular}{|c|c|c|c|}
\hline & Paragraphenzeichen & Initialen & Nummerierungen / Varia \\
\hline 16,18 & $141(>)$ & 108,141 & \\
\hline 16,19 & $141(>)$ & 19,124 & \\
\hline 16,20 & $4(>), 141(>), 154(\cdot \dot{*})$ & 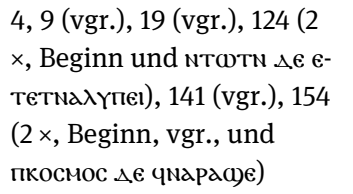 & „Amen-Wort““ 23: 4 \\
\hline 16,21 & $141(>)$ & $19,141,154$ (vgr.) & \\
\hline 16,22 & $141(>)$ & $\begin{array}{l}\text { 19, 108, } 115 \text { (bei мnмссо), } \\
141\end{array}$ & \\
\hline 16,23 & $\begin{array}{l}\text { pbo (bei гамнм), } 4 \text { (> bei 2a- } \\
\text { мнм), } 141 \text { (> bei гамнм) }\end{array}$ & 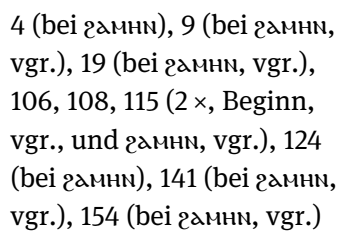 & „Amen-Wort“ 24: 4 \\
\hline 16,24 & & 108 & \\
\hline 16,25 & pbo, 9 & $\begin{array}{l}9 \text { (vgr.), } 19 \text { (vgr.), 108, } 115 \text { ( } 2 \\
\times \text {, Beginn und oүn oүoүnor, } \\
\text { vgr.), } 124 \text { (vgr.) }\end{array}$ & \\
\hline 16,26 & & 108, 115 (vgr.), 124 & \\
\hline 16,27 & & 115 & \\
\hline 16,28 & & $108,115,124$ & \\
\hline 16,29 & No. $931(\dot{*})$ & $\begin{array}{l}\text { 19, 105, } 115 \text { ( } 2 \times \text {, Beginn und } \\
\text { аүळ мгхє), No. } 931 \text { (vgr.) }\end{array}$ & \\
\hline 16,30 & $87(>)$ & *87 (vgr.), 115 & Abschnitt 36: 115 (m2) \\
\hline 16,31 & pbo, *154 (※) & $\begin{array}{l}\text { 19, } 103 \text { (vgr.), 108, } 115 \\
\text { (vgr.), *154 }\end{array}$ & \\
\hline 16,32 & & 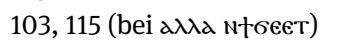 & \\
\hline 16,33 & $131($ einzige $\dot{*}$ ) & $\begin{array}{l}9 \text { (vgr.), 19, } 103(2 \times, \text { Be- } \\
\text { ginn, vgr. und oүNTHTN, } \\
\text { vgr.), 108, } 115(2 \times, \text { Beginn, } \\
\text { vgr., und oүNTHTN), 124, } 131\end{array}$ & $\begin{array}{l}\{\text { can.?: } 131(\text { einzige } \dot{*} \text { ) }\} ; \text { Ab- } \\
\text { schnitt 36: } 108\end{array}$ \\
\hline 17,1 & $\begin{array}{l}1(>), 4(>), 5(>/ \odot), 10(\dot{*}), \\
124(\dot{*}), 131(\dot{*})\end{array}$ & $\begin{array}{l}\text { 4, 5, 9, } 10 \text { (vgr.), } 19 \text { (vgr.), } \\
103 \text { (vgr.), } 124 \text { (vgr.), 131, } \\
115,124\end{array}$ & $\begin{array}{l}\text { can. 153/X: 131; Abschnitt } \\
\text { 36: } 124\end{array}$ \\
\hline \multicolumn{4}{|l|}{17,2} \\
\hline 17,3 & & 108 & \\
\hline 17,4 & $\begin{array}{l}103(\dot{*}), 124(\dot{*}), 140(\dot{*}), \\
154(\dot{*}), \text { *No. } 931(\dot{*})\end{array}$ & $\begin{array}{l}19 \text { (vgr.), 103, 108, 115, } 124 \\
\text { (vgr.), 140, 154, *No. } 931\end{array}$ & $\begin{array}{l}\text { Glosse: } 103(\overline{1 .} \text { eXN пПатPl- } \\
\text { архнс сеүнрос } \overline{I \Lambda})\end{array}$ \\
\hline 17,5 & & 115 & \\
\hline 17,6 & $103(\dot{x})$ & 103,115 & \\
\hline 17,7 & $\{\mathrm{pbo}\}$ & 115 & \\
\hline 17,8 & & 108, 115 (bеi аүпıстеүє) & \\
\hline 17,9 & 108 & 108 & \\
\hline
\end{tabular}




\begin{tabular}{|c|c|c|c|}
\hline & Paragraphenzeichen & Initialen & Nummerierungen / Varia \\
\hline \multicolumn{4}{|l|}{17,10} \\
\hline 17,11 & 108 (bei амок $\Delta €$ eINнY) & 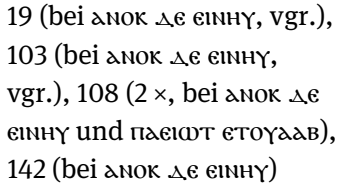 & \\
\hline 17,12 & & $\begin{array}{l}\text { 19, 103, } 142 \text { (bеi єІмнтеı п- } \\
\text { с)нрє, vgr.) }\end{array}$ & \\
\hline 17,13 & $140(\cdot \dot{x})$ & 19 (vgr.), 103 (vgr.), 124, 140 & \\
\hline 17,14 & 4, $131(\div), * 140(\cdot \dot{*})$ & $\begin{array}{l}4,108,131, * 140 \text { (nur } * \dot{*} \text { er- } \\
\text { halten) }\end{array}$ & \\
\hline 17,15 & 140 & 140 & \\
\hline 17,16 & $131(\div)$ & 131, 142 (bei ката өє, vgr.) & \\
\hline \multicolumn{4}{|l|}{17,17} \\
\hline 17,18 & $131(\div), 140$ & $124,126,131,140$ & \\
\hline \multicolumn{4}{|l|}{17,19} \\
\hline 17,20 & & 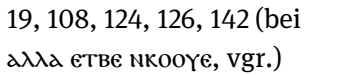 & \\
\hline 17,21 & $\begin{array}{l}140 \text { (bei хєкас ерє } \\
\text { пкосмос) }\end{array}$ & 140 (bei хекас єрє пкосмос) & \\
\hline 17,22 & & 124 & \\
\hline \multicolumn{4}{|l|}{17,23} \\
\hline 17,24 & $\begin{array}{l}126 \text { (über und unter Ini.), } \\
140\end{array}$ & $19,108,124,126,140$ & \\
\hline 17,25 & 140 & 19,140 & \\
\hline 17,26 & $\begin{array}{l}126 \text { (bei aүo on } \\
\text { †NגOYon24) }\end{array}$ & & \\
\hline 18,1 & $\begin{array}{l}\text { pbo, } 1(>), 4(>), 5(>/ \odot), 10 \\
(\cdot \dot{*}), 124(\cdot \dot{*}), 140(\cdot \dot{*})\end{array}$ & $\begin{array}{l}\text { 4, 5, } 9 \text { (vgr.), } 10 \text { (vgr.), } 19 \\
\text { (vgr.), 108, } 124 \text { (vgr.), } 126 \\
\text { (bei nal nтерєчхоor, vgr.), } \\
127 \text { (Spuren von Ini. und } \\
. \dot{x}), 140\end{array}$ & $\begin{array}{l}\text { Abschnitt 36: } 10 \text { (m2); Ab- } \\
\text { schnitt 37: 108, } 124 \text { (Titel } \\
\text { clœn, auch liturgische Ru- } \\
\text { brik); †: } 124 \text { (m2, doppeltes } \\
\text { Andreaskreuz, cf. 18,6) }\end{array}$ \\
\hline 18,2 & & 108,124 & \\
\hline 18,3 & 140 & $108,124,140$ & \\
\hline 18,4 & 140 & $\begin{array}{l}9 \text { (vgr.), 19, 108, 124, 140, } \\
235\end{array}$ & \\
\hline 18,5 & $74(>)$ & $\begin{array}{l}19 \text { (bei nєчд2є } \Delta \epsilon), * 74 \text { (bei } \\
\text { мєчд2є } \Delta \epsilon), 108 \text { (bei nєчд2є } \\
\Delta \epsilon), 124 \text { (bei мєчд2є } \Delta \epsilon \text { ) }\end{array}$ & \\
\hline 18,6 & & 127,234 & 粶: 124 (m2, Andreaskreuz) \\
\hline 18,7 & 140 & 19 (vgr.), 108, 124, 140 & \\
\hline 18,8 & pbo, 140 (bei єс)хє амок) & $\begin{array}{l}74,127,140 \text { (bei ec)xe a- } \\
\text { Noк), } 234\end{array}$ & \\
\hline 18,9 & & & $\begin{array}{l}\text { Zitat } \rightarrow »: 4 ;\{\text { *?: } 124 \text { (cf. } \\
18,15)\}\end{array}$ \\
\hline
\end{tabular}




\begin{tabular}{|c|c|c|c|}
\hline & Paragraphenzeichen & Initialen & Nummerierungen / Varia \\
\hline 18,10 & & $\begin{array}{l}9 \text { (vgr.), 108, 124, 127, } 235 \\
\text { (vgr.) }\end{array}$ & $\begin{array}{l}\text { Glossen: } 127 \text { (пмаү мпа- } \\
\lambda<\epsilon>\text { ктүр und пाмд rechts } \\
\text { der Kol.) }\end{array}$ \\
\hline 18,11 & 140 & $19,108,124,127,140$ & \\
\hline 18,12 & 140 & $108,124,127,140,235$ (vgr.) & \\
\hline 18,13 & $141(>)$ & $141($ bei мєпа)ом $\triangle \epsilon)$ & \\
\hline 18,14 & $141(>)$ & $108,124,127,141$ & \\
\hline 18,15 & $\begin{array}{l}10(\cdot \dot{*}), 140,141(2 \times, \text {, Be- } \\
\text { ginn und пмдөнтнс } \Delta € \\
\text { етммаҮ }), 260\end{array}$ & $\begin{array}{l}\text { 9, } 10 \text { (vgr.), } 19 \text { (vgr.), 108, } \\
\text { 124, 127, 140, } 141 \text { ( } 2 \times, \text { Be- } \\
\text { ginn und пмдөнтнс } \triangle є е \text { стм- } \\
\text { маү), } 235,260\end{array}$ & $\begin{array}{l}\text { Abschnitt 37: } 10(\mathrm{~m} 2) \text {; Ab- } \\
\text { schnitt 38: 108, 124, 260; }+ \text { : } \\
124 \text { (Andreaskeuz und } \\
\text { Kreuz, wohl } * \text { bei } 18,9 \text { ) }\end{array}$ \\
\hline 18,16 & $141(>)$ & $108,127,141,235$ & \\
\hline 18,17 & $141(>)$ & $19,127,141,235$ & \\
\hline 18,18 & $\begin{array}{l}141(2 \times, \text {, Beginn und пєт- } \\
\text { poc } \Delta \epsilon), 260(2 \times \text {, Beginn } \\
\text { und пєтрос } \Delta €)\end{array}$ & $\begin{array}{l}19,108,127,141(2 \times, \text { Be- } \\
\text { ginn und пєтрос } \Delta €), 235 \text {, } \\
260(2 \times, \text { Beginn und } \\
\text { пєтрос } \Delta €)\end{array}$ & \\
\hline 18,19 & 140 & $108,124,127,140,235$ & \\
\hline 18,20 & $\begin{array}{l}\text { pbo, } 141 \text { ( }>\text { bei anok NoYo- } \\
\text { (10)), } 260\end{array}$ & 19 (vgr.), 127, 260 & \\
\hline \multicolumn{4}{|l|}{18,21} \\
\hline 18,22 & pbo, $141(>), 260$ & $19,108,127,141,235,260$ & \\
\hline 18,23 & pbo (unsicher), 260 & $19,108,235,260$ & \\
\hline 18,24 & 140 & $\begin{array}{l}19 \text { (vgr.), 108, 127, 140, } 235 \\
\text { (vgr.) }\end{array}$ & \\
\hline 18,25 & & 9 (vgr.), 103, 108, 235 & \\
\hline 18,26 & 140 & $103,108,127,140,235$ & \\
\hline 18,27 & & & $\begin{array}{l}\text { Glosse: } 140 \text { (оүळ2 єво } \lambda \text { м- } \\
\text { пеІма) }\end{array}$ \\
\hline 18,28 & $\begin{array}{l}1(>), 4(>), 5(>/ \odot), 103(\cdot \dot{*}), \\
127,140(\cdot \dot{*})\end{array}$ & 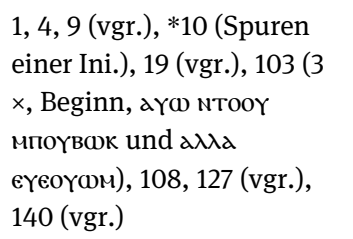 & $\begin{array}{l}\text { Glossen: } 127 \text { (піма und п- } \\
\text { NaҮ мс)орп); } \$: 140 \text { (бопє } \\
\text { ом єтєхпсо, cf. 19,14) }\end{array}$ \\
\hline 18,29 & 140 & 19 (vgr.), 127, 140 & \\
\hline 18,30 & pbo & 19 (vgr.), 108, 127 & \\
\hline 18,31 & & $19,108,139$ & \\
\hline 18,32 & 103 & & \\
\hline 18,33 & 140 & $\begin{array}{l}19 \text { (vgr.), 103, 108, 127, 140, } \\
155 \text { (vgr.) }\end{array}$ & \\
\hline 18,34 & pbo, 260 & $\begin{array}{l}19,108,124,139,155 \text { (vgr.), } \\
260\end{array}$ & \\
\hline 18,35 & pbo, 260 & 103, 124, 139, 155 (vgr.), 260 & \\
\hline
\end{tabular}




\begin{tabular}{|c|c|c|c|}
\hline & Paragraphenzeichen & Initialen & Nummerierungen / Varia \\
\hline 18,36 & $\begin{array}{l}\text { pbo, } 83(2 \times \text {, Beginn, und } \\
\text { TenoY } \Delta \epsilon \text { mit }>), 116 \text { (bei } \\
\text { TenoY } \Delta \epsilon \text {, Versbeginn } \\
\text { verloren), 140, } 260\end{array}$ & 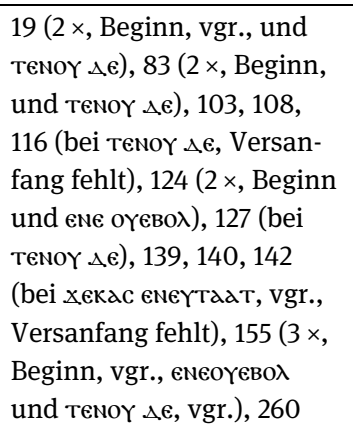 & $\begin{array}{l}\text { \{453: Ini. und } \div \text { bei ene oү- } \\
\text { єво } \lambda \text {, doch nur Zitat in } \\
\text { Homilie oder ähnlichem\} }\end{array}$ \\
\hline 18,37 & 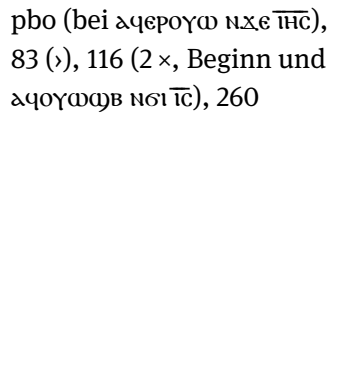 & 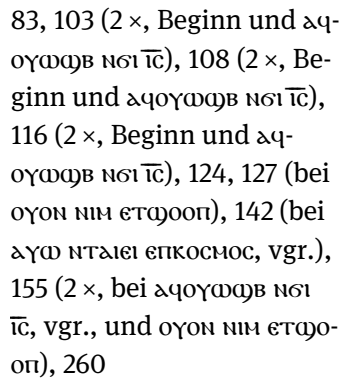 & \\
\hline 18,38 & 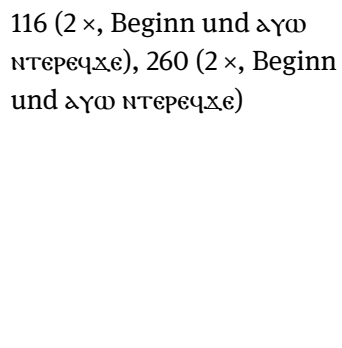 & 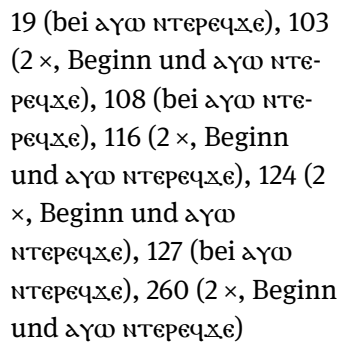 & \\
\hline 18,39 & $127(\div)$ & $103,108,127,155$ & \\
\hline 18,40 & & $\begin{array}{l}\text { 103, } 127 \text { (bеi вараввас } \triangle \epsilon \\
\text { меоүсоонє пє), } 155\end{array}$ & \\
\hline 19,1 & $\begin{array}{l}\text { pbo, } 10(\stackrel{\times}{*}), 103,116(\dot{*}) \\
127(\div)\end{array}$ & $\begin{array}{l}10 \text { (vgr.), } 19 \text { (vgr.), } 103 \\
\text { (vgr.), 108, } 116 \text { (vgr.), 124, } \\
\text { 127, } 139 \text { (vgr.), } 155 \text { (vgr.) }\end{array}$ & $\begin{array}{l}\text { Abschnitt 39: *10 (m2, nur } \\
\text { Spuren), 108, 116, 124, } 139 \\
\text { (m2), 155; Glosse: } 116 \text { (г und } \\
\text { Punkte, m2, Karfreitag, } 3 . \\
\text { Tagesstunde); Glosse: } 127 \\
\text { (піма und 21 по)омте) }\end{array}$ \\
\hline 19,2 & & 155 (bei ayt 210ळq) & \\
\hline 19,3 & & & \\
\hline 19,4 & & 19, 103, 124, 127, 155 (vgr.) & \\
\hline 19,5 & 140 & $\begin{array}{l}19 \text { (vgr.), 103, 110, 124, 127, } \\
140\end{array}$ & \\
\hline
\end{tabular}




\begin{tabular}{|c|c|c|c|}
\hline & Paragraphenzeichen & Initialen & Nummerierungen / Varia \\
\hline 19,6 & 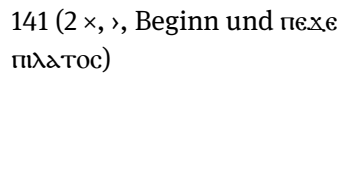 & 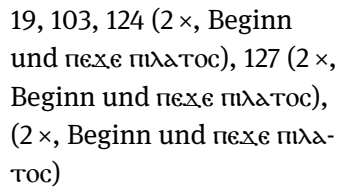 & \\
\hline 19,7 & & $\begin{array}{l}103 \text { (bei аүळ ката пемnо- } \\
\text { мос), 124, 127, } 140 \text { (einzige } \\
\text { Ini. ohne } \dot{*} \text { ), } 141\end{array}$ & \\
\hline 19,8 & 103,116 & $\begin{array}{l}\text { 19, 103, 108, 116, 124, } 127 \\
\text { (bei ıс } \triangle є \text { мпечоүсовв) }\end{array}$ & \\
\hline \multicolumn{4}{|l|}{19,9} \\
\hline 19,10 & & $\begin{array}{l}\text { 19, } 103(2 \times, \text { Beginn und } \\
\text { oүw+ тezoycla }), 108,127\end{array}$ & \\
\hline 19,11 & pbo, $141(>)$ & $\begin{array}{l}19 \text { (vgr.), 103, 108, 124, 127, } \\
141\end{array}$ & \\
\hline 19,12 & $\begin{array}{l}116(\div \text { und } \odot \text { bei nıoY } \Delta a l \\
\Delta \epsilon)\end{array}$ & 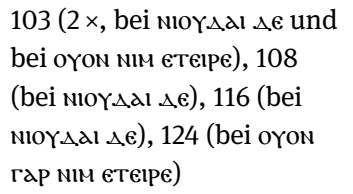 & \\
\hline 19,13 & $10(\div, \mathrm{m} 2), * 116(\dot{*})$ & $\begin{array}{l}10,19,103,108, * 116 \text { (vgr.), } \\
124,127\end{array}$ & $\begin{array}{l}\text { \$: } 116 \text { ( } \varsigma \text { und Punkte, m2, } \\
\text { Karfreitag, 6. Tagesstunde); } \\
\text { Glosse: } 140 \text { ([c] ]ạ గ̣ıma) }\end{array}$ \\
\hline 19,14 & 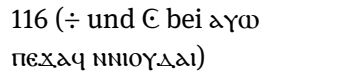 & 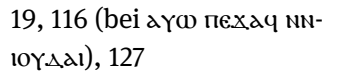 & Glosse: 140 ([оүळг] е़пाмд) \\
\hline 19,15 & $\{$ pbo $\}$ & 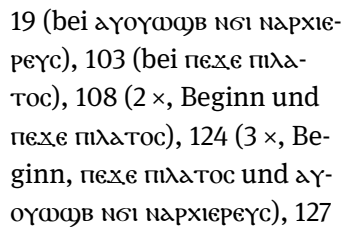 & $\begin{array}{l}\text { Glosse: } 140 \text { (бопє ом м̄пıмд } \\
\text { [єтммд]Y Nтехпү!тє, Kar- } \\
\text { freitag, } 6 . \text { Tagesstunde) }\end{array}$ \\
\hline 19,16 & $\begin{array}{l}103(\dot{*} \text { bei nтоoҮ } \triangle \epsilon), 116 \\
(\dot{*})\end{array}$ & 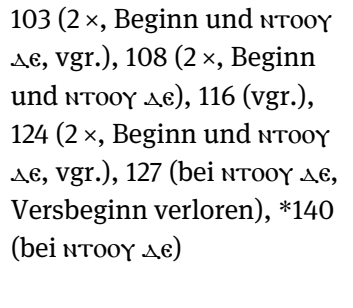 & 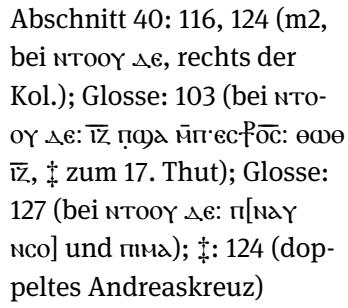 \\
\hline 19,17 & $116(\dot{x}$ bei aqєı) & 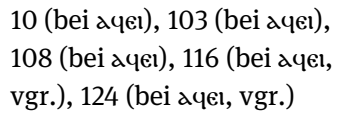 & $\begin{array}{l}\text { Abschnitt 40: 108, } 155 \text { (bei } \\
\text { ayel) }\end{array}$ \\
\hline 19,18 & & $\begin{array}{l}103 \text { (bei oүa ncd пeicd), } 116 \\
\text { (bei oүd Ncd пеıсd) }\end{array}$ & \\
\hline 19,19 & $116(\div$ und $\odot)$ & $\begin{array}{l}19 \text { (vgr.), 103, 108, 116, 124, } \\
127,155 \text { (bei мєчснг } \triangle є \text { ероч) }\end{array}$ & \\
\hline
\end{tabular}




\begin{tabular}{|c|c|c|c|}
\hline & Paragraphenzeichen & Initialen & Nummerierungen / Varia \\
\hline 19,20 & & $\begin{array}{l}\text { 124, } 127 \text { (bеi аүळ мечснг), } \\
155\end{array}$ & \\
\hline 19,21 & & 103, 124, 155 (vgr.), 199 & \\
\hline 19,22 & & 103, 108, 124, 155, 199 (vgr.) & *: 10 (Doppelkreuz, m2) \\
\hline 19,23 & $116(\div$ und $* \odot), 140$ & 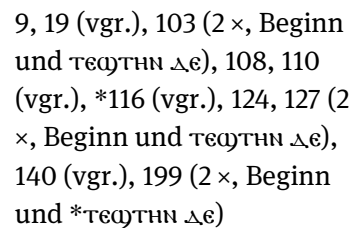 & Zit. $\rightarrow \div: 199$ (? oder $*$ ) \\
\hline 19,24 & $\begin{array}{l}110(\div \text { bei хекас еретегрд- } \\
\phi н), 199(\div)\end{array}$ & 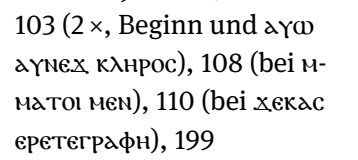 & $\begin{array}{l}\text { Zit. } \rightarrow »: 1,4,9,110 \text { (durch } \\
\div \div)\end{array}$ \\
\hline 19,25 & $\begin{array}{l}4(>/ \odot), 103,116(\div \text { und } \dot{\times}) \text {, } \\
199(\div)\end{array}$ & $\begin{array}{l}4 \text { (vgr.), } 19 \text { (vgr.), 103, } 116 \\
\text { (vgr.), 121, } 127 \text { (vgr.), } 155 \\
\text { (vgr.), } 199\end{array}$ & $\begin{array}{l}\text { Zit. } \rightarrow \div: 199 \text { (? oder } * \text { ?, am } \\
\text { Versende); Glosse: } 127 \text { ( } \text { xl- }^{-} \\
\text {үІте und пाмд, } * \text { ) }\end{array}$ \\
\hline 19,26 & $110(\div), 199(\div)$ & $19,103,108,110, * 199$ & \\
\hline \multicolumn{4}{|l|}{19,27} \\
\hline 19,28 & $\begin{array}{l}4(>), 103,116(\div \text { und } \div) \text {, } \\
199(>)\end{array}$ & $\begin{array}{l}\text { 4, 9, 19, 103, 108, } 116 \text { (vgr.), } \\
155 \text { (vgr.), } 199\end{array}$ & $\begin{array}{l}\text { \$: } 116 \text { (ө und Punkte, m2, } \\
\text { Karfreitag, 9. Tagesstunde); } \\
\text { Abschnitt 41: } 108\end{array}$ \\
\hline 19,29 & & $\begin{array}{l}103 \text { (bei оүспоггос } \triangle \varepsilon), 127, \\
199\end{array}$ & \\
\hline 19,30 & & 19, 103 (bei пєхач хє), 127 & \\
\hline 19,31 & $116(\div$ und $\odot)$ & $103,108,116$ & $\begin{array}{l}\text { †: } 116 \text { ( } \overline{\mathrm{d}} \text { und Punkte, m2, } \\
\text { 11. Tagestunde des Karfrei- } \\
\text { tags) }\end{array}$ \\
\hline 19,32 & $199(\odot)$ & 19 (vgr.), 121, 127, 199 (vgr.) & \\
\hline 19,33 & & $103,127,155$ & \\
\hline 19,34 & & 103,127 & \\
\hline 19,35 & $199(\div)$ & & \\
\hline 19,36 & & $103,108,127$ & Zit. $\rightarrow »: 1,4,5$ \\
\hline 19,37 & & & Zitat $\rightarrow »: 1,4,5$ \\
\hline 19,38 & 4, $10(\dot{*})$ & $\begin{array}{l}\text { 4, 9, } 10 \text { (vgr.), } 19 \text { (vgr.), 103, } \\
108103 \text { ( } 2 \times \text {, Beginn und } \\
\text { аүळ дчкגач), 127, } 155 \text { (vgr.) }\end{array}$ & $\begin{array}{l}\text { \{Keph. 17: } 9 \text { (kein Verweis } \\
\text { am Text, nur Keph.-Titel)\}; } \\
\text { \{Keph. 18: } 10 \text { (rezent); }\} \text { Ab- } \\
\text { schnitt 41: } 10 \text { (m2), } 155 \\
\text { (m2); Glosse: } 127 \text { (пnaY } \\
\text { NpoY<२८>) }\end{array}$ \\
\hline 19,39 & & $19,103,127$ & \\
\hline 19,40 & & 127 & \\
\hline 19,41 & 4 & 4 (vgr.), 19, 103 & Glosse: No. 948 (сал пाмд) \\
\hline 19,42 & & & \\
\hline
\end{tabular}




\begin{tabular}{|c|c|}
\hline & Paragraphenzeichen \\
\hline 20,1 & 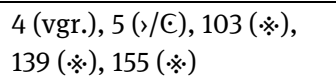 \\
\hline \multicolumn{2}{|l|}{20,2} \\
\hline \multicolumn{2}{|l|}{20,3} \\
\hline \multicolumn{2}{|l|}{20,4} \\
\hline \multicolumn{2}{|l|}{20,5} \\
\hline \multicolumn{2}{|l|}{20,6} \\
\hline \multicolumn{2}{|l|}{20,7} \\
\hline \multicolumn{2}{|l|}{20,8} \\
\hline \multicolumn{2}{|l|}{20,9} \\
\hline 20,10 & $\{\mathrm{pbo}\}$ \\
\hline 20,11 & $4(>), 103,143(\odot / × \dot{*})$ \\
\hline \multicolumn{2}{|l|}{20,12} \\
\hline 20,13 & $\begin{array}{l}116(\div \text { und } \odot \text { bei пехас NaY } \\
\times e), 369(\dot{*})\end{array}$ \\
\hline 20,14 & $116(\div$ und $\odot)$ \\
\hline 20,15 & $\begin{array}{l}116(2 \times \text {, Beginn und nexac } \\
\text { Nay) }\end{array}$ \\
\hline
\end{tabular}

$\begin{array}{ll}20,16 & \text { pbo, } 369(\dot{x}) \\ 20,17 & 369(\dot{*} \cdot)\end{array}$

19 (bei aүळ дчєı Nœ)орп, vgr.)

4, 5, 9 (vgr.), 19 (vgr.), 103, Keph. 18: 9; Abschnitt 42: 139 (vgr.), 155, 182 (vgr.), 139 (m2), 155, 182; Glosse: *No. 948 103 (паl Ф0) NTKYPIakн мпво $\lambda$ єво入, Ende bei 20,18)

19, 103

103

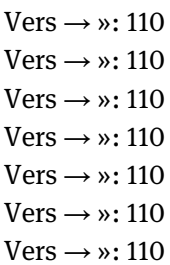

Хac NaY Xe), 369

19, 103 (vgr.), 116

19, $115(2 \times$, bei TH $\Delta \in$ und mexac Nay, Beginn verloren), 116 ( $2 \times$, Beginn und nexac Nay) 19, 103, 115, 369 (vgr.) 19, $103(2 \times$, Beginn und вок $\triangle \epsilon), 115(2 \times$, Beginn und вळк $\triangle$ e), 369 (vgr.)

20,18

9 (vgr.), 19, 115, 116

*: 103 (Glosse: (1)d)

20,19 $4($ (〉), *10 (※) $), 103(\dot{*}), 116$ $(\dot{*}), 182(\dot{*}), 369(\dot{*})$ 115 (vgr.), 116 (vgr.), 182 (2 der Kol., m2), 116 (m2), 182, 369; †: 103 (па Фа) мп.. $\times$, Beginn und aqeı Nol $\overline{\mathrm{Ic}})$, 369 (vgr.)

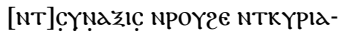
19 (vgr.), 115 (bei aүpac)e $\mathrm{KH})$ $\Delta \epsilon$, Versbeginn verloren)

20,21 \{pbo\}, $4(>), 116$

4, 19, 103, 115, 116

$20,22 \quad 369(\div$ und $\dot{*})$

19 (bei аүш пехач), 103, 115, 369

20,23116

20,24 pbo , 4 ( (), 9, $103(\stackrel{*}{*}), 116$ (※), $369(\stackrel{*}{*})$

115, 116

4 (vgr.), 9 (vgr.), 19 (vgr.),

Abschnitt 44: 115 (m2), 116, 103, 115 (2×, Beginn und 182; †: 103 (ерепӓ̈ Фа) ....,

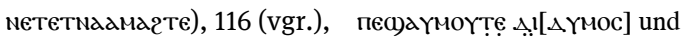
182 (vgr.), 268, 369 (vgr.) andactacic $\bar{x} \bar{\varphi} \bar{\varphi})$ 


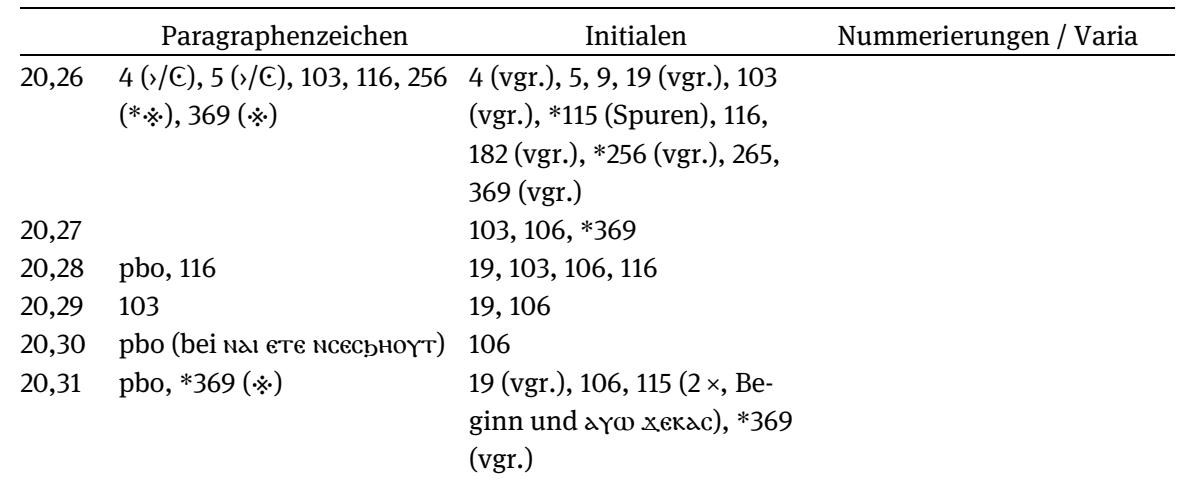

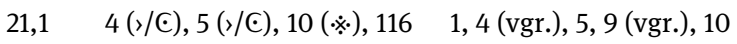
(※), 271

21,2
21,3

21,3

21,4

21,5

21,6

$21,7 \quad 116(\div$ und $\odot$ bei cIman oє пєтрос)

21,8 (pbo)

21,9 pbo, $116(\div$ und $\odot)$

21,10

21,11

21,12

$21,13 * 271$

$21,14 \quad 131(\div)$

21,15 $4($ ( ), $10(\dot{*}), 116(\dot{*}), 131$ (2 $\times$, Beginn und nexay nay

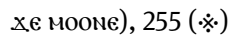

21,16 $131(2 \times$, Beginn, $€$, und $\pi \epsilon-$ xay nay $x \in$ moone, $\div$ )

21,17 $131(2 \times$, Beginn, $€$, und пехе $\overline{\mathrm{IC}}$ NaY, $\div$ )

(vgr.), 19 (vgr.), 115 (2×,

Beginn und entayoron24), 116 (vgr.), 136, 182 (vgr.),

271

115

19, 182 (vgr.)

19

19, 106, 182 (bei nтоoҮ $\triangle$ e, vgr.)

$19(2 \times$, Beginn und стмюN бє петрос), 116 (bei сімюм бе петрос)

19

19

19

$19, * 271$

4, 10 (vgr.), 116 (vgr.), 131 (2

$\times$, Beginn und пехач naч хє мооме), 182 (vgr.), 255

(vgr.)

19, $131(2 \times$, Beginn und пехау nay $x \in$ moone)

9 (vgr.), $131 \quad$ can. 225/IX: 131

Abschnitt 45: 10 (m2), *115

(Spuren einer Zahl, m2),

116, 182 


\begin{tabular}{|c|c|c|c|}
\hline & Paragraphenzeichen & Initialen & Nummerierungen / Varia \\
\hline 21,18 & $\begin{array}{l}\text { pbo (unsicher), } 4 \text { ( (), } 5 \text { (Ho- } \\
\text { rizontalstrich), } 131(\div)\end{array}$ & $\begin{array}{l}4 \text { (vgr.), } 9 \text { (vgr.), } 103(2 \times \text {, } \\
\text { Beginn und готаN } \Delta \epsilon), 131 \text {, } \\
182 \text { (bei готаN } \Delta \epsilon, \text { vgr.) }\end{array}$ & $\begin{array}{l}\text { „Amen-Wort“ 25: 4; can. } \\
\text { 231/IX, } 131\end{array}$ \\
\hline 21,19 & $\begin{array}{l}131(2 \times \text {, Beginn, } \div \text {, und } \\
\text { Nтереч } х є \text { пал, } \div)\end{array}$ & 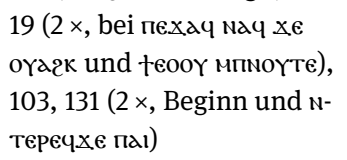 & \\
\hline 21,20 & & $\begin{array}{l}\text { 65, } 103(2 \times \text {, Beginn und пал } \\
\text { Nтגyno } \times 4), 237\end{array}$ & \\
\hline 21,21 & $131(\div)$ & $19,103,131$ & \\
\hline 21,22 & $131(\div)$ & 103,131 & \\
\hline 21,23 & $131(\div)$ & $\begin{array}{l}103(2 \times, \text { Beginn und } \\
\text { NTay } \times \text { ooc }), 131\end{array}$ & \\
\hline 21,24 & $131(\div)$ & 19,131 & \\
\hline 21,25 & $131(\div)$ & 19,131 & $\begin{array}{l}\text { Glossen: } 103 \text { (liturgische } \\
\text { Rubriken und griechisch } \\
20,1-7^{\text {lac }},=\text { Osterlesung) }\end{array}$ \\
\hline
\end{tabular}

